\title{
Nucleon resonances in Compton scattering
}

\author{
Gernot Eichmann ${ }^{1}$ and G. Ramalho ${ }^{2}$ \\ ${ }^{1}$ CFTP, Instituto Superior Técnico, Universidade de Lisboa, 1049-001 Lisboa, Portugal \\ ${ }^{2}$ Laboratório de Física Teórica e Computacional-LFTC, \\ Universidade Cruzeiro do Sul, 01506-000, São Paulo, SP, Brazil
}

(Received 18 June 2018; published 28 November 2018)

\begin{abstract}
We calculate the nucleon resonance contributions to nucleon Compton scattering, including all states with $J^{P}=1 / 2^{ \pm}$and $J^{P}=3 / 2^{ \pm}$where experimental data for their electromagnetic transition form factors exist. To this end, we construct a tensor basis for the Compton scattering amplitude based on electromagnetic gauge invariance, crossing symmetry and analyticity. The corresponding Compton form factors provide a Lorentz-invariant description of the process in general kinematics, which reduces to the static and generalized polarizabilities in the appropriate kinematic limits. We derive the general forms of the offshell nucleon-to-resonance transition vertices that implement electromagnetic and spin-3/2 gauge invariance, which automatically also defines onshell transition form factors that are free of kinematic constraints. We provide simple fits for those form factors, which we use to analyze the resulting Compton form factors and extract their contributions to the nucleon's polarizabilities. Apart from the $\Delta(1232)$, the resonance contributions to the scalar and spin polarizabilites are very small, although the $N(1520)$ could play a role for the proton's magnetic polarizability.
\end{abstract}

DOI: 10.1103/PhysRevD.98.093007

\section{INTRODUCTION}

Compton scattering on the nucleon encodes a multitude of interesting physical applications. It is the process $\gamma^{*} N \rightarrow \gamma^{*} N$, sketched in Fig. 1, where either of the photons can be real or virtual. Compton scattering probes the electromagnetic structure of the nucleon and therefore the quarks inside. On the one hand, it encodes the nucleon's polarizabilities which test its response to an external electromagnetic field. Ongoing efforts with chiral effective field theory, dispersion relations and other approaches aim to determine the proton's and neutron's scalar and spin polarizabilities [1-5]. On the other hand, virtual Compton scattering (VCS), where one photon is virtual and the other is real, provides access to the nucleon's generalized polarizabilities [1,6-9]. Deeply virtual Compton scattering (DVCS) is the primary tool to extract the nucleon's generalized parton distributions (GPDs) [10-13]; and the forward limit, where the momentum transfer vanishes, is experimentally accessible in deep inelastic scattering and relates the Compton amplitude with the nucleon structure functions and PDFs.

Published by the American Physical Society under the terms of the Creative Commons Attribution 4.0 International license. Further distribution of this work must maintain attribution to the author(s) and the published article's title, journal citation, and DOI. Funded by SCOAP .
Also the integrated Compton amplitude is of interest. The diagram where the two photons couple to a lepton encodes the two-photon exchange (TPE) corrections to electromagnetic form factors. These are believed to be responsible for the difference in the proton's $G_{E} / G_{M}$ measurements, because the Rosenbluth separation method is sensitive to TPE effects whereas the polarization transfer experiments are not [14-16]. However, at present it still remains to be clarified which parts of the Compton amplitude cause the difference. TPE contributions also enter in the proton radius puzzle although so far the effect appears to be too small by an order of magnitude to explain the discrepancy [17-22].

At the hadronic level, the Compton amplitude can be split into "elastic" Born terms and an "inelastic" structure part as in Fig. 1. In principle the Born terms are determined by the nucleon electromagnetic form factors, whereas the oneparticle irreducible (1PI) structure part encodes the structure information such as polarizabilities. The latter probes the spectrum of hadrons: in terms of its singularity structure, it contains intermediate nucleon resonances in the $s$ and $u$ channels such as the $\Delta(1232)$ resonance, meson exchanges in the $t$ channel, and vector-meson poles for timelike photon virtualities. These are accompanied by multiparticle cuts in the various channels, which come from $N \pi, N \pi \pi, \pi \pi \ldots$ loops and are directly accessible in effective field theory approaches. For example, it is well known that the $\Delta$ resonance provides a large contribution to the magnetic polarizability which is counteracted by pion loops, thus 

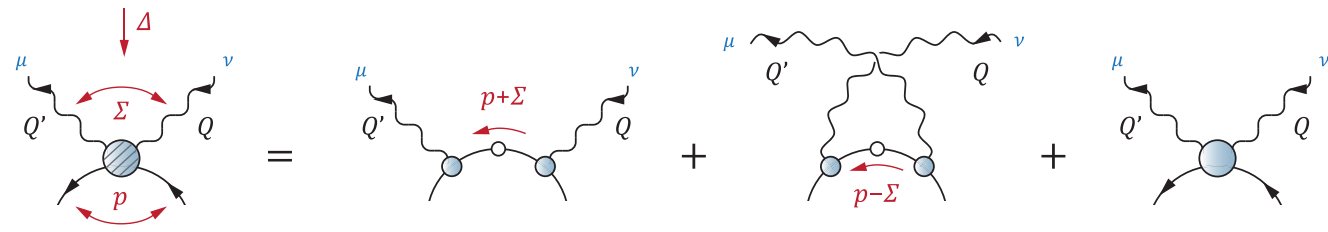

FIG. 1. Separation of the nucleon Compton amplitude into Born terms and a 1PI structure part.

leading to the picture of a "paramagnetic quark core" that is cancelled by its "diamagnetic pion cloud" [5,23-25].

On the other hand, handbag dominance in DVCS attributes the dynamics in Compton scattering to an interaction of the photons with the perturbative quarks inside the nucleon. It is then understood that the hadronic description should be applied at low energies whereas the microscopic approach is appropriate when $Q^{2}$ is large. Still, it is desirable to connect these two regimes by a common underlying formulation. Such an approach in terms of nonperturbative quark and gluon degrees of freedom (d.o.f.) has been formulated [26-28] but it is not the topic of the present work. Here we aim for a more modest goal, namely to establish a connection in terms of common amplitudes to describe the process in arbitrary kinematics.

In principle Compton scattering is completely specified by 18 Lorentz-invariant functions [29], which are probed in different kinematic limits by the experimental processes mentioned above. The purpose of this paper is to make a step towards connecting these limits by providing a tensor basis based on electromagnetic gauge invariance, crossing symmetry and analyticity. This leads to a set of 18 Compton form factors (CFFs) which depend on four Lorentzinvariant variables and which are free of kinematic constraints. In the limit where all variables vanish these are related to the nucleon's polarizabilities, in VCS they are connected to the generalized polarizabilities and in the forward limit to the nucleon's structure functions. Each CFF has certain characteristics: the nucleon Born terms contribute to only a few of them, as well as the $t$-channel meson poles; and only certain subsets of them survive in the forward limit, in RCS or in VCS.

Following the approach by Bardeen, Tung and Tarrach $[29,30]$, similar tensor bases have been employed for specific applications such as low-energy VCS [31,32] or scalar Compton scattering [33,34]. Here we provide the detailed basis construction for a spin-1/2 target in general kinematics, using a procedure that differs from Refs. [29,30] and allows one to better track the occurrence or absence of kinematic singularities. It is still true that kinematic singularities cannot be avoided in certain limits [35], but this does not affect the 18 CFFs in general or the limits of RCS, VCS and the forward limit where direct measurements are possible.

As a practical application we work out the CFF contributions from intermediate $s$ - and $u$-channel nucleon resonances, which enter in the process through their electromagnetic transition form factors. The $\Delta(1232)$ contribution to the nucleon's polarizabilities is known [9,36,37], but in view of a precision determination of polarizabilities it is still desirable to understand the impact of higher resonances, which can also play a role in TPE [38-40]. In the last decade significant progress has been made in measuring the electrocouplings of nucleon resonances through meson electroproduction in a wide $Q^{2}$ range [41,42]. In addition to the $\Delta(1232)$ resonance, the electromagnetic transitions are now relatively well known also for the Roper resonance $N(1440)$, the nucleon's tentative parity partner $N(1535)$, and the $N(1520)$ resonance. First data for higher-lying resonances have been accumulated in two-pion production [43-45] and more results are underway with the Jefferson Lab $12 \mathrm{GeV}$ program.

The fact that the resonances in Compton scattering are offshell creates additional complications. Electromagnetic gauge invariance and spin-3/2 gauge invariance for RaritaSchwinger particles $[46,47]$, which ensures the absence of the spin-1/2 background in matrix elements, induce further constraints on the offshell transition vertices. Here we derive the most general structure for the $J^{P}=1 / 2^{ \pm}$and $J^{P}=3 / 2^{ \pm}$transition amplitudes that are compatible with these constraints. As a result, their implementation in Compton scattering automatically ensures the absence of spurious contributions.

Moreover, these expressions also determine the most general forms of the onshell nucleon-to-resonance electromagnetic transition currents which are free of kinematic constraints. One obtains two form factors $F_{1,2}\left(Q^{2}\right)$ in the $J=1 / 2$ case and three form factors $F_{1,2,3}\left(Q^{2}\right)$ for $J=3 / 2$ and higher spin, which are kinematically independent so that all their singularities and momentum dependencies are of dynamical origin. The experimental data are usually discussed in terms of helicity amplitudes or multipole form factors $[41,48,49]$ which are neither free of kinematics nor satisfy the offshell constraints. Here we provide simple fits to the experimental data for all available resonances in terms of the constraint-free form factors $F_{i}\left(Q^{2}\right)$. Those parametrizations we finally implement in the Compton amplitude to calculate the CFFs in the entire kinematic domain.

The paper is organized as follows. In Sec. II we establish our notation, discuss the kinematic regions in terms of four Lorentz-invariant variables, provide the tensor basis for the Compton amplitude and investigate different kinematic limits. In Sec. III we illustrate the situation for scalar 
Compton scattering. In Sec. IV we discuss the nucleon Born terms, together with the offshell nucleon-photon vertex that enters there, in some detail and work out the corresponding CFFs. In Secs. V and VI we apply the same procedure to derive the $J=1 / 2$ and $J=3 / 2$ resonance contributions, respectively, and in Sec. VII we provide our fits for their transition form factors. The resulting CFFs and polarizabilities are discussed in Sec. VIII. We summarize in Sec. IX.

Several Appendices serve the purpose of making our calculations as transparent as possible for practitioners. We use a Euclidean metric for practical convenience but with the formulas in Appendix A the transcription between Euclidean and Minkowski conventions should be straightforward. In Appendix B we explain the tensor basis derivation for the Compton amplitude in detail; we provide relations between our CFFs and the amplitude conventions in some kinematic limits used in the literature; and we investigate the consequences of breaking gauge invariance for the nucleon Born term. Appendix $\mathrm{C}$ gives some details on spin-3/2 Lagrangians, and in Appendix D we collect the relations between the resonance transition form factors and helicity amplitudes employed in the literature.

\section{COMPTON AMPLITUDE}

\section{A. Kinematics}

The onshell nucleon Compton amplitude with virtual photons has the form

$$
\mathcal{M}^{\mu \nu}\left(p, Q^{\prime}, Q\right)=\frac{e^{2}}{m} \bar{u}\left(p_{f}\right) \Gamma^{\mu \nu}\left(p, Q^{\prime}, Q\right) u\left(p_{i}\right),
$$

where $e^{2}=4 \pi \alpha_{\mathrm{em}}, m$ is the nucleon mass, $Q$ and $Q^{\prime}$ are the incoming and outgoing photon four-momenta, $p_{i}$ and $p_{f}$ are the initial and final on-shell nucleon momenta $\left(p_{i}^{2}=p_{f}^{2}=-m^{2}\right)$, and $p=\left(p_{i}+p_{f}\right) / 2$ is the average nucleon momentum (see Fig. 1). $u\left(p_{i}\right)$ and $\bar{u}\left(p_{f}\right)$ are nucleon spinors satisfying the Dirac equation; they are eigenspinors of the positive-energy projectors

$$
\Lambda_{+}\left(p_{f}\right)=\frac{-i \not p_{f}+m}{2 m}, \quad \Lambda_{+}\left(p_{i}\right)=\frac{-i \not \not_{i}+m}{2 m}
$$

with $\Lambda_{+}\left(p_{i}\right) u\left(p_{i}\right)=u\left(p_{i}\right)$ and $\bar{u}\left(p_{f}\right) \Lambda\left(p_{f}\right)=\bar{u}\left(p_{f}\right)$. It is then more convenient to work with the Dirac matrix-valued Compton amplitude

$$
\Gamma^{\mu \nu}\left(p, Q^{\prime}, Q\right)=\Lambda_{+}\left(p_{f}\right)\left[\sum_{i=1}^{18} c_{i} X_{i}^{\mu \nu}\right] \Lambda_{+}\left(p_{i}\right),
$$

where the spinors are replaced with the projectors. The Compton amplitude is constructed from 18 dimensionless Compton form factors (CFFs) $c_{i}$ which depend on four kinematical invariants, together with 18 Lorentz-covariant basis tensors $X_{i}^{\mu \nu}\left(p, Q^{\prime}, Q\right)$.

We will alternatively use two sets of four-vectors, $\left\{p, Q, Q^{\prime}\right\}$ and $\{p, \Sigma, \Delta\}$, with the relations

$$
\begin{aligned}
& p=\frac{1}{2}\left(p_{i}+p_{f}\right), \\
& \Sigma=\frac{1}{2}\left(Q+Q^{\prime}\right),
\end{aligned} \quad \Delta=Q-Q^{\prime}=p_{f}-p_{i}
$$

and

$$
\begin{array}{ll}
p_{i}=p-\frac{\Delta}{2}, & Q=\Sigma+\frac{\Delta}{2}, \\
p_{f}=p+\frac{\Delta}{2}, & Q^{\prime}=\Sigma-\frac{\Delta}{2} .
\end{array}
$$

With the constraints $p_{i}^{2}=p_{f}^{2}=-m^{2}$, the process is characterized by four Lorentz invariants. We work with the dimensionless variables ${ }^{1}$

$$
\begin{aligned}
\eta_{+} & =\frac{Q^{2}+Q^{\prime 2}}{2 m^{2}}, \quad \eta_{-}=\frac{Q \cdot Q^{\prime}}{m^{2}}, \quad \omega=\frac{Q^{2}-Q^{\prime 2}}{2 m^{2}}, \\
\lambda & =-\frac{p \cdot \Sigma}{m^{2}}=-\frac{p \cdot Q}{m^{2}}=-\frac{p \cdot Q^{\prime}}{m^{2}}
\end{aligned}
$$

and vice versa

$$
\begin{aligned}
& \left\{\begin{array}{c}
Q^{2} \\
Q^{\prime 2}
\end{array}\right\}=\Sigma^{2}+\frac{\Delta^{2}}{4} \pm \Sigma \cdot \Delta=m^{2}\left(\eta_{+} \pm \omega\right), \\
& Q \cdot Q^{\prime}=\Sigma^{2}-\frac{\Delta^{2}}{4}=m^{2} \eta_{-},
\end{aligned}
$$

so that the CFFs in Eq. (2.3) are dimensionless functions $c_{i}\left(\eta_{+}, \eta_{-}, \omega, \lambda\right)$. The variables $\eta_{+}$and $\eta_{-}$are even under photon crossing and charge conjugation, whereas $\omega$ and $\lambda$ switch signs [see Eq. (2.19) below]. Below we employ a tensor basis that is invariant under both operations, so that the CFFs can depend on $\omega$ and $\lambda$ only quadratically.

\footnotetext{
${ }^{1}$ Introducing new symbols for these variables provides a compact notation but also has the following advantage: we use Euclidean conventions throughout this work, but since Lorentzinvariant scalar products differ from their Minkowski counterparts only by minus signs these variables are the same in Minkowski space if one defines them as

$$
\begin{aligned}
\eta_{+}=-\frac{q^{2}+q^{\prime 2}}{2 m^{2}}, & \eta_{-}=-\frac{q \cdot q^{\prime}}{m^{2}}, \\
\omega=-\frac{q^{2}-q^{\prime 2}}{2 m^{2}}, & \lambda=\frac{\tilde{p} \cdot q}{m^{2}}=\frac{\tilde{p} \cdot q^{\prime}}{m^{2}},
\end{aligned}
$$

where $\tilde{p}, q$ and $q^{\prime}$ are the Minkowski momenta corresponding to $p, Q$ and $Q^{\prime}$. In that way all relations between Lorentz-invariant (but also Lorentz-covariant) quantities, such as the CFFs given in Tables IV, VI and IX, are identical in Euclidean and Minkowski conventions; see Appendix A for more details.
} 

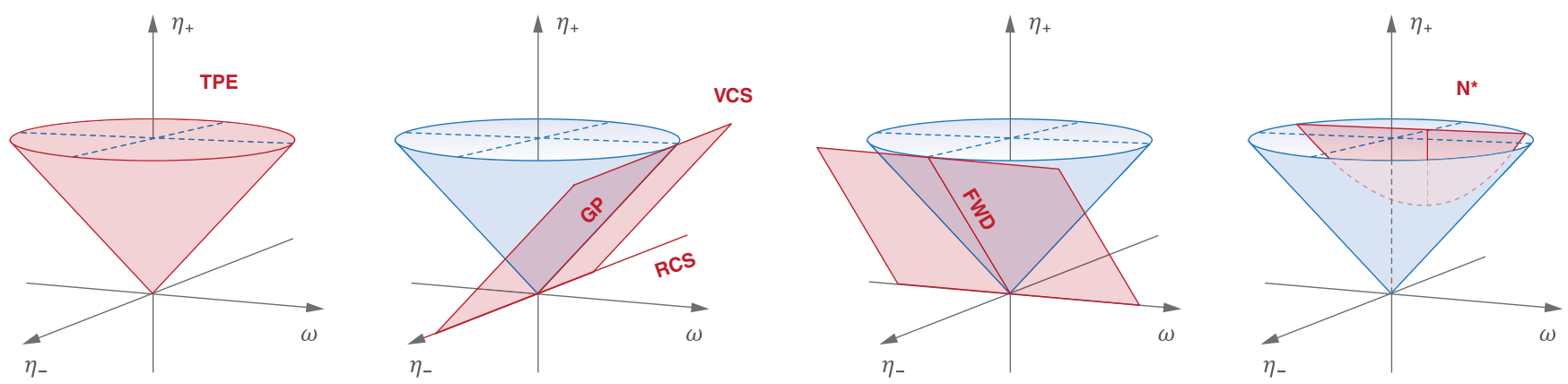

FIG. 2. Compton scattering in the variables $\eta_{+}, \eta_{-}$and $\omega$. The interior of the cone contributes to two-photon exchange (TPE). Real Compton scattering (RCS) lives on the $\eta_{-}$axis and virtual Compton scattering (VCS) on the plane $\eta_{+}=\omega$. The boundary of the cone contains the doubly-virtual forward limit (FWD) at $t=0\left(\eta_{+}=\eta_{-}\right)$and the VCS limit where the generalized polarizabilities are defined (GP, $\eta_{+}=\omega$ and $\eta_{-}=0$ ). Inside the cone, nucleon resonances appear at $\eta_{-}=-\delta$.

The variables $\eta_{+}, \eta_{-}$and $\omega$ admit a simple geometrical understanding of the phase space. Defining the momentum transfer as

$$
t=\frac{\Delta^{2}}{4 m^{2}}=\frac{\eta_{+}-\eta_{-}}{2}
$$

(which differs by $-4 m^{2}$ from the usual definition), then for $t>0$ the region that one must integrate over in order to calculate two-photon exchange (TPE) contributions to observables forms a cone around the $\eta_{+}$direction, which is shown in the leftmost panel in Fig. 2. This is so because $\Sigma^{\mu}$ is the integration momentum and the integration region is subject to the constraints

$$
\sigma>0, \quad-1<Z<1, \quad-1<Y<1
$$

where $\sigma, Z$ and $Y$ are the hyperspherical variables from Eq. (A8) with the Lorentz-invariant definition

$$
\sigma=\frac{\Sigma^{2}}{m^{2}}, \quad Z=\hat{\Sigma} \cdot \hat{\Delta}, \quad Y=\hat{p} \cdot \widehat{\Sigma_{\perp}} .
$$

Here, a hat denotes a normalized four-momentum (e.g., $\hat{\Sigma}=\Sigma / \sqrt{\Sigma^{2}}$ ) and the subscript $\perp$ stands for a transverse projection with respect to the total momentum transfer $\Delta$. These variables are related to the ones in Eq. (2.6) via

$$
\begin{gathered}
\sigma=\frac{\eta_{+}+\eta_{-}}{2}, \quad Z=\frac{\omega}{\sqrt{\eta_{+}^{2}-\eta_{-}^{2}}}, \\
\lambda=\frac{Y}{2} \sqrt{\omega^{2}+\eta_{-}^{2}-\eta_{+}^{2}} \sqrt{1+\frac{2}{\eta_{+}-\eta_{-}}} .
\end{gathered}
$$

With $t>0$ the first two constraints in (2.9) entail

$$
-\eta_{+}<\eta_{-}<\eta_{+}, \quad \omega^{2}+\eta_{-}^{2}<\eta_{+}^{2}
$$

which defines the cone in Fig. 2. Because of

$$
\eta_{+} \pm \omega=\frac{1}{m^{2}}\left\{\begin{array}{c}
Q^{2} \\
Q^{\prime 2}
\end{array}\right\}, \quad \frac{\eta_{+} \pm \eta_{-}}{2}=\left\{\begin{array}{c}
\sigma \\
t
\end{array}\right\}
$$

the opposite sides of the cone in the $\left\{\omega, \eta_{+}\right\}$plane define the axes of $Q^{2}$ and $Q^{\prime 2}$, whereas in the $\left\{\eta_{-}, \eta_{+}\right\}$plane the cone is bounded by the axes for $\sigma$ and $t$. Because $Y \in$ $(-1,1)$ is real inside the cone, the crossing variable $\lambda$ must become imaginary due to Eqs. (2.12) and (2.13).

In Fig. 2 we show the various kinematic limits:

(i) Real Compton scattering (RCS): $Q^{2}=Q^{\prime 2}=$ $0 \Rightarrow \eta_{+}=\omega=0$.

(ii) Virtual Compton scattering (VCS): $Q^{\prime 2}=0 \Rightarrow$ $\eta_{+}=\omega$.

(iii) Generalized polarizabilities: $Q^{\prime \mu}=0 \Rightarrow \eta_{+}=\omega$, $\eta_{-}=\lambda=0$.

(iv) Doubly-virtual forward limit: $\Delta^{\mu}=0 \Rightarrow \eta_{+}=\eta_{-}$, $\omega=0$.

(v) Static polarizabilities: $\eta_{+}=\eta_{-}=\omega=\lambda=0$.

In the $3 \mathrm{D}$ plots the static polarizabilities are defined at the origin of the coordinate system; the forward amplitudes and generalized polarizabilities live on the boundary of the cone where $t=0$ or $Q^{\prime 2}=0$, respectively; the RCS limit is defined along the $\eta_{-}$axis outside of the cone; and the VCS limit defines the plane $Q^{\prime 2}=0$.

The nucleon resonance poles at $s=m_{R}^{2}$ and $u=m_{R}^{2}$, where $s$ and $u$ are the Mandelstam variables and $m_{R}$ is the mass of the resonance, are more difficult to visualize because they also depend on the crossing variable $\lambda$ :

$\left\{\begin{array}{l}s \\ u\end{array}\right\}=-(p \pm \Sigma)^{2}=m^{2}\left[1-\left(\eta_{-} \mp 2 \lambda\right)\right]=m_{R}^{2}$,

with $\lambda=(s-u) /\left(4 m^{2}\right)$. Taking also into account the resonance width, $m_{R}^{2} \rightarrow m_{R}^{2}-i m_{R} \Gamma_{R}$, and defining

$$
\delta=\frac{m_{R}^{2}-m^{2}}{m^{2}}, \quad \gamma=\frac{m_{R} \Gamma_{R}}{2 m^{2}},
$$

the condition for a pole becomes 


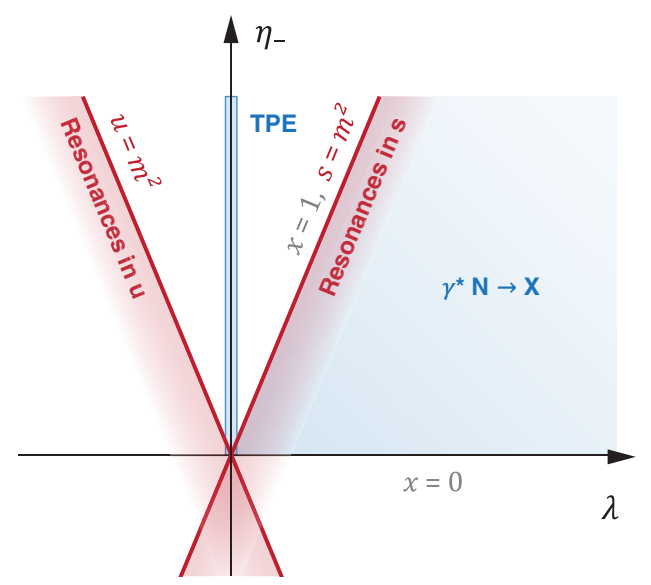

FIG. 3. Kinematics in forward Compton scattering in the variables $\eta_{-}$and $\lambda$.

$$
\eta_{-}= \pm 2 \lambda-\delta+2 i \gamma
$$

Figure 3 illustrates the situation in the forward limit, where the two remaining variables $\eta_{-}=\eta_{+}=Q^{2} / \mathrm{m}^{2}$ and $\lambda$ define the Mandelstam plane. The forward CS amplitude is of special interest because the optical theorem relates its imaginary part to the total photoabsorption cross section $\gamma^{*} N \rightarrow X$ and thus to the nucleon's structure functions. The physical region of that process is where the Bjorken variable $x=\eta_{-} /(2 \lambda)$ takes values $0 \leq x \leq 1$. The nucleon resonances appear at fixed $s$ and $u$, starting with the nucleon poles at $s=m^{2}$ and $u=m^{2}$ (corresponding to $\eta_{-}= \pm 2 \lambda$ ). The resonance regions are indicated by the red shaded areas in the plot, where at larger $s$ and $u$ the resonances are eventually washed out. In addition, one has branch cuts from multiparticle production: the right-hand cut at $s \geq\left(m+m_{\pi}\right)^{2}$, which starts at the pion production threshold and extends to infinity, the left-hand cut at $u \geq\left(m+m_{\pi}\right)^{2}$, plus further cuts in the timelike region where $\eta_{-}$is negative.

Except for the nucleon Born poles and branch cuts, the CFFs are analytic functions in the physical sheet, given that they are defined through an appropriate tensor basis which does not introduce additional kinematic singularities. Since their imaginary parts along the cuts are known from the cross section data, one can exploit Cauchy's formula to determine the CFFs everywhere in the complex $\lambda$ plane via (subtracted) dispersion relations. Except for the subtraction functions, which can be determined in chiral effective field theory (see e.g., the reviews [3,5]), the forward CS amplitude is then in principle fully determined by experimental data.

The TPE region is the interior of the cone where the crossing variable $\lambda$ is imaginary. In the forward limit Eq. (2.12) becomes $\lambda=i Y \sqrt{\eta_{-}}$, so that the remnant of the cone is the domain $\operatorname{Re}(\lambda)=0$ and $|\operatorname{Im}(\lambda)| \leq \sqrt{\eta_{-}}$along the imaginary $\lambda$ axis, as indicated in Fig. 3. On the other hand, for small values of $\lambda$ the CFFs can be expanded in powers of $\lambda^{2}$. The $Q^{2}$-dependent forward polarizabilities are accordingly defined as the coefficients in a low-energy expansion:

$c_{i}\left(\eta_{-}, \lambda\right)=c_{i}^{\mathrm{Born}}\left(\eta_{-}, \lambda\right)+c_{i}^{\mathrm{Pol}}\left(\eta_{-}, \lambda=0\right)+\mathcal{O}\left(\lambda^{2}\right)$,

where the Born contributions are singular for $\lambda= \pm \eta_{-} / 2$ and the remaining pieces absorb all structure effects.

Because the nucleon resonance locations only depend on $\eta_{-}$and $\lambda$, the Mandelstam plane has the same form as in Fig. 3 also in general kinematics, such as e.g., in RCS and VCS, although the respective physical regions are different. The interior of the cone always corresponds to imaginary $\lambda$, so that the condition (2.17) becomes $\eta_{-}=-\delta$ and $\operatorname{Im}(\lambda)=\mp \gamma$. Thus, for negative values of $\eta_{-}$the resonance poles can appear in the TPE integration region and must be properly taken care of. This is illustrated by the vertical plane in the rightmost panel of Fig. 2 for an exemplary resonance. The poles of the nucleon itself $(\delta=\gamma=0)$ intersect with the cone in the limit $\eta_{-}=\lambda=0$. In the case of VCS $\left(\eta_{+}=\omega\right)$ this is just the limit where the generalized polarizabilities are defined (second panel in Fig. 2), so that an extraction of polarizabilities requires a sensible subtraction of the nucleon poles contained in the nucleon Born terms.

\section{B. Tensor basis}

The extraction of CFFs requires a suitable tensor basis. While in principle the tensor decomposition is arbitrary, the choice of basis matters in practice. Compton scattering is characterized by 18 CFFs $c_{i}\left(\eta_{+}, \eta_{-}, \omega, \lambda\right)$, cf. Eq. (2.3), and thus it is desirable to construct a basis where these functions become as simple as possible:

(i) Gauge invariance must be properly implemented, which reduces the number of CFFs from 32 to 18 . Below we write down a basis where transversality is manifest.

(ii) The Compton amplitude is invariant under photon crossing and charge conjugation:

$$
\begin{aligned}
& \Gamma^{\mu \nu}\left(p, Q^{\prime}, Q\right) \stackrel{!}{=} \Gamma^{\nu \mu}\left(p,-Q,-Q^{\prime}\right), \\
& \Gamma^{\mu \nu}\left(p, Q^{\prime}, Q\right) \stackrel{!}{=} C \Gamma^{\nu \mu}\left(-p,-Q,-Q^{\prime}\right)^{T} C^{T},
\end{aligned}
$$

where $C=\gamma^{4} \gamma^{2}$ is the charge-conjugation matrix and the superscript $T$ denotes a matrix transpose. Implementing these properties already at the level of the basis elements simplifies the discussion because the resulting CFFs can depend on the variables $\omega$ and $\lambda$ only quadratically: $c_{i}\left(\eta_{+}, \eta_{-}, \omega^{2}, \lambda^{2}\right)$.

(iii) To make the CFFs dimensionless we divide the basis tensors by powers of the nucleon mass $m$.

(iv) The CFFs should be free of kinematic singularities; analyticity then implies that their only singularities 
are physical poles and cuts. In Fig. 2 the dominant poles are: the $s$ - and $u$-channel nucleon and nucleon resonance poles; the lowest vector-meson poles in $Q^{2}$ and $Q^{\prime 2}$, which live on planes parallel to the VCS plane (and their mirror planes with $\omega \rightarrow-\omega$ ); and the lowest $t$-channel meson poles on planes parallel to the forward plane outside of the cone. Being free of kinematic effects has several practical advantages; it can simplify the momentum dependence of the CFFs, as their dependence on the four variables $\eta_{+}$, $\eta_{-}, \lambda$ and $\omega$ effectively often collapses into a onedimensional dependence on $\eta_{+}$. The absence of kinematic dependencies in the CFFs is tied to using a "minimal" basis, which is characterized by having no kinematic singularities and featuring the lowest possible powers in the photon momenta. Such bases have been frequently used in the literature following the works by Bardeen, Tung and Tarrach $[29,30]$.

Without reference to the separation into Born and 1PI terms in Fig. 1, one can generally decompose the on-shell Compton amplitude into three contributions:

$$
\Gamma^{\mu \nu}=\Gamma_{\mathrm{G}}^{\mu \nu}+\Gamma_{\perp}^{\mu \nu}+\Gamma_{\perp \perp}^{\mu \nu},
$$

which are distinguished by their transversality properties. $\Gamma_{\perp \perp}^{\mu \nu}$ is transverse with respect to both photon momenta,

$$
Q^{\prime \mu} \Gamma_{\perp \perp}^{\mu \nu}=0, \quad \Gamma_{\perp \perp}^{\mu \nu} Q^{\nu}=0,
$$

whereas $\Gamma_{\perp}^{\mu \nu}$ is subject to the weaker constraint

$$
Q^{\prime \mu} \Gamma_{\perp}^{\mu \nu} Q^{\nu}=0
$$

and the remaining "gauge part" $\Gamma_{\mathrm{G}}^{\mu \nu}$ is not transverse.

The physical Compton amplitude is gauge invariant, so it must satisfy (2.21) and thus only $\Gamma_{\perp \perp}^{\mu \nu}$ survives. The full amplitude depends on 32 independent Lorentz-Dirac tensors, 18 of which belong to $\Gamma_{\perp \perp}^{\mu \nu}, 12$ to $\Gamma_{\perp}^{\mu \nu}$ and two to $\Gamma_{\mathrm{G}}^{\mu \nu}$. In the main text we will only consider the physical, transverse part $\Gamma_{\perp \perp}^{\mu \nu}$ which depends on 18 tensors. However, to quantify a potential loss of gauge invariance it is also useful to work out the remaining nontransverse terms $\Gamma_{\mathrm{G}}^{\mu \nu}$ and $\Gamma_{\perp}^{\mu \nu}$, which is done in Appendix B 3 .

The derivation of the 18 transverse tensors is straightforward and sketched in Appendix B. One starts from a set of 32 linearly independent elementary tensors, the $K_{i}^{\mu \nu}$ in Table XV, and applies the constraints (2.21) such that no kinematic singularities are introduced. In practice this means eliminating 14 CFFs without any division by kinematic factors, i.e., without introducing denominators that depend on $\eta_{+}, \eta_{-}, \lambda^{2}, \omega^{2}$, etc. Fortunately, in the case of Compton scattering this is possible and thus the procedure automatically generates a minimal basis.

The resulting 18 transverse basis elements $X_{i}^{\mu \nu}$ are lengthy combinations of the $K_{i}^{\mu \nu}$ and given in Table XVI
TABLE I. Transverse basis for the nucleon Compton amplitude. $\mathrm{F}_{i, j}$ and $\mathrm{G}_{i, j}$ are defined in Eq. (2.24) and the explicit expressions for the first few tensors are given in Eq. (2.26).

\begin{tabular}{llll}
\hline \hline$n$ & Basis element & $n$ & \multicolumn{1}{c}{ Basis element } \\
\hline 2 & $X_{1}=\mathrm{F}_{1,6}$ & 3 & $X_{10}=\mathrm{F}_{1,21}-\frac{1}{4} \mathrm{~F}_{1,34}+2 \mathrm{~F}_{1,6}$ \\
2 & $X_{2}=\frac{1}{2} \mathrm{~F}_{1,1}$ & 3 & $X_{11}=\mathrm{F}_{6,33}+\frac{1}{4} \mathrm{~F}_{2,33}$ \\
4 & $X_{3}=\mathrm{F}_{1,2}$ & 3 & $X_{12}=\mathrm{F}_{1,33}$ \\
4 & $X_{4}=\mathrm{F}_{2,6}$ & 5 & $X_{13}=\mathrm{F}_{2,33}$ \\
4 & $X_{5}=\mathrm{F}_{1,9}$ & 5 & $X_{14}=\mathrm{F}_{1,27}+2 \mathrm{~F}_{1,22}$ \\
2 & $X_{6}=\frac{1}{4} \mathrm{G}_{1,1}$ & 3 & $X_{15}=\frac{1}{\lambda^{2}} \mathrm{~F}_{9,33}$ \\
3 & $X_{7}=\frac{1}{\lambda \omega} \mathrm{G}_{1,24}$ & 5 & $X_{16}=\frac{1}{\lambda^{2}} \mathrm{~F}_{10,33}$ \\
5 & $X_{8}=\frac{\omega}{\lambda} \mathrm{G}_{1,23}$ & 4 & $X_{17}=\mathrm{F}_{1,23}$ \\
5 & $X_{9}=\frac{\omega}{\lambda} \mathrm{G}_{1,25}$ & 6 & $X_{18}=\mathrm{F}_{1,24}$ \\
\hline \hline
\end{tabular}

in the Appendix, but they can be written in a compact way using the definitions

$$
t_{A}^{\mu \alpha \beta}=\delta^{\mu \beta} A^{\alpha}-\delta^{\mu \alpha} A^{\beta}, \quad \varepsilon_{A}^{\mu \alpha \beta}=\gamma_{5} \varepsilon^{\mu \alpha \beta \lambda} A^{\lambda} .
$$

These are the lowest-dimensional Lorentz tensors that are linear in the momenta and transverse without introducing kinematic singularities. $t_{A}^{\mu \alpha \beta}$ is transverse to the momentum $A^{\mu}$, whereas $\varepsilon_{A}^{\mu \alpha \beta}$ is transverse in all Lorentz indices: $A^{\mu} t_{A}^{\mu \alpha \beta}=0, A^{\mu} \varepsilon_{A}^{\mu \alpha \beta}=0$, etc. With their help we define Compton basis tensors of the form

$$
\begin{aligned}
\mathrm{F}_{i, j}^{\mu \nu} & =\frac{1}{2 m^{2}} t_{Q^{\prime}}^{\mu \alpha \rho} t_{Q}^{\nu \beta \sigma}\left\{K_{i}^{\alpha \beta}, K_{j}^{\rho \sigma}\right\}, \\
\mathrm{G}_{i, j}^{\mu \nu} & =\frac{1}{2 m^{2}}\left(t_{Q^{\prime}}^{\mu \alpha \rho} \varepsilon_{Q}^{\nu \beta \sigma}+\varepsilon_{Q^{\prime}}^{\mu \alpha \rho} t_{Q}^{\nu \beta \sigma}\right)\left\{K_{i}^{\alpha \beta}, K_{j}^{\rho \sigma}\right\}
\end{aligned}
$$

which are dimensionless and manifestly transverse with respect to $Q^{\prime \mu}$ and $Q^{\nu}$. They define our transverse basis in Table I, with the $K_{i}^{\mu \nu}$ given in Table XV.

To arrive at more explicit expressions, we further define

$$
\begin{aligned}
t_{A B}^{\mu \nu} & =t_{A}^{\mu \alpha \nu} B^{\alpha}=A \cdot B \delta^{\mu \nu}-B^{\mu} A^{\nu}, \\
\varepsilon_{A B}^{\mu \nu} & =\varepsilon_{A}^{\mu \alpha \nu} B^{\alpha}=\gamma_{5} \varepsilon^{\mu \nu \alpha \beta} A^{\alpha} B^{\beta}
\end{aligned}
$$

where $A^{\mu}, B^{\mu}$ stand for the four-vectors $p^{\mu}, Q^{\mu}$ and $Q^{\prime \mu}$. These expressions are quadratic in the momenta and also manifestly transverse: $t_{A B}^{\mu \nu}=t_{B A}^{\nu \mu}$ is transverse to $A^{\mu}$ and $B^{\nu}$ whereas $\varepsilon_{A B}^{\mu \nu}=\varepsilon_{B A}^{\nu \mu}$ is transverse to $A$ and $B$ in both Lorentz indices. With their help the Compton tensors in Table I take the form 


$$
\begin{aligned}
& X_{1}^{\mu \nu}=\frac{1}{m^{4}} t_{Q^{\prime} p}^{\mu \alpha} t_{p Q}^{\alpha \nu}, \quad X_{2}^{\mu \nu}=\frac{1}{m^{2}} t_{Q^{\prime} Q}^{\mu \nu}, \\
& X_{3}^{\mu \nu}=\frac{1}{m^{4}} t_{Q^{\prime} Q^{\prime}}^{\mu \alpha} t_{Q Q}^{\alpha \nu}, \quad X_{4}^{\mu \nu}=\frac{1}{m^{6}} t_{Q^{\prime} Q^{\prime}}^{\mu \alpha} p^{\alpha} p^{\beta} t_{Q Q}^{\beta \nu}, \\
& X_{5}^{\mu \nu}=\frac{\lambda}{m^{4}}\left(t_{Q^{\prime} Q^{\prime}}^{\mu \alpha} t_{p Q}^{\alpha \nu}+t_{Q^{\prime} p}^{\mu \alpha} t_{Q Q}^{\alpha \nu}\right), \quad X_{6}^{\mu \nu}=\frac{1}{m^{2}} \varepsilon_{Q^{\prime} Q}^{\mu \nu}, \\
& X_{7}^{\mu \nu}=\frac{1}{i m^{3}}\left(t_{Q^{\prime} Q^{\prime}}^{\mu \alpha} \varepsilon_{\gamma Q}^{\alpha \nu}-\varepsilon_{Q^{\prime} \gamma}^{\mu \alpha} t_{Q Q}^{\alpha \nu}\right), \\
& X_{8}^{\mu \nu}=\frac{\omega}{i m^{3}}\left(t_{Q^{\prime} Q^{\prime}}^{\mu \alpha} \varepsilon_{\gamma Q}^{\alpha \nu}+\varepsilon_{Q^{\prime} \gamma}^{\mu \alpha} t_{Q Q}^{\alpha \nu}\right),
\end{aligned}
$$

etc. For $X_{7}^{\mu \nu}$ and $X_{8}^{\mu \nu}$ we have extended the definition (2.25) to also include $\gamma$-matrices [see Eq. (A15) for the definition of the triple commutator]:

$$
\varepsilon_{\gamma A}^{\mu \nu}=\gamma_{5} \varepsilon^{\mu \nu \alpha \beta} \gamma^{\alpha} A^{\beta}=\frac{1}{6}\left[\gamma^{\mu}, \gamma^{\nu}, \mathbb{A}\right]=\frac{1}{4}\left\{\left[\gamma^{\mu}, \gamma^{\nu}\right], \mathbb{A}\right\} .
$$

Note that the denominators of $X_{7,8,9,15,16}^{\mu \nu}$ in Table I do not lead to kinematic singularities because they are matched by corresponding factors from the $K_{i}^{\mu \nu}$ which enter in Eq. (2.24).

By construction, all basis elements $X_{i}^{\mu \nu}$ and $K_{i}^{\mu \nu}$ are even under photon crossing and charge conjugation, i.e., they satisfy the requirements of Eq. (2.19):

$$
\begin{aligned}
& X_{i}^{\mu \nu}\left(p, Q^{\prime}, Q\right) \stackrel{!}{=} X_{i}^{\nu \mu}\left(p,-Q,-Q^{\prime}\right), \\
& X_{i}^{\mu \nu}\left(p, Q^{\prime}, Q\right) \stackrel{!}{=} C X_{i}^{\nu \mu}\left(-p,-Q,-Q^{\prime}\right)^{T} C^{T} .
\end{aligned}
$$

The systematic (anti) symmetrization and use of commutators ensure that all tensors are either even or odd under these operations, and with appropriate prefactors $\lambda, \omega$ and $\lambda \omega$ they become symmetric. Because these are also the symmetries of the Compton amplitude, the resulting CFFs are even in $\lambda$ and $\omega$ so that they can depend on these variables only quadratically. Bose symmetry and charge conjugation amount to a permutation-group symmetry $S_{2} \times S_{2}$ and therefore the CFFs corresponding to Table I are permutation-group singlets.

For a given tensor $X_{i}^{\mu \nu}$ in Table I, the number $n$ counts the powers in the photon momenta. It can be read off from the definitions (2.24) and the $K_{i}^{\mu \nu}$ in Table XV: each fourmomentum $Q^{\prime \mu}, Q^{\mu}$ as well as the Lorentz invariant $\lambda$ contribute $n=1$, whereas $\omega, \eta_{+}$and $\eta_{-}$contribute $n=2$. In principle this is useful for the construction of minimal bases characterized by the lowest overall photon momentum powers [50]: collect all linearly independent tensors with $n=2$, then proceed to $n=3$, etc.

For example, for Compton scattering on a scalar particle, which only involves the tensors $X_{1 \ldots .}^{\mu \nu}$, the minimality is tied to the alignment $n=\{2,2,4,4,4\}$. On the one hand, it is not possible to find more than two tensors with $n=2$ unless one divides by kinematic variables, which leads to kinematic singularities in the basis elements. On the other hand, replacing tensors in the set by others with higher $n$ introduces kinematic singularities in the $\mathrm{CFFs}$, because those higher momentum powers must be matched by respective denominators in the CFFs. For example, in Tarrach's original basis [29] the following tensor with $n=6$ appears:

$$
\mathrm{F}_{1,10}^{\mu \nu}=\frac{\lambda \omega}{m^{4}}\left(t_{Q^{\prime} Q^{\prime}}^{\mu \alpha} t_{p Q}^{\alpha \nu}-t_{Q^{\prime} p}^{\mu \alpha} t_{Q Q}^{\alpha \nu}\right)
$$

Noting that the resulting basis is not minimal, it was subsequently exchanged with $X_{4}^{\mu \nu}=\mathrm{F}_{2,6}^{\mu \nu}$ which is still linearly independent but only has $n=4$. (In Tarrach's notation $X_{4}^{\mu \nu} \propto \tau_{19}^{\mu \nu}$ and the bracket above is identical to $-\tau_{5}^{\mu \nu}$, cf. Table XVII in Appendix B 1.) Thus, only those transverse bases that are free of kinematic singularities and satisfy $n=\{2,2,4,4,4\}$ are minimal and guarantee the absence of kinematic dependencies in the CFFs. [As a caveat, see the discussion below Eq. (2.44).]

Unfortunately, for the $X_{6 \ldots 18}^{\mu \nu}$ the counting is obscured by the contraction with the on-shell projectors in (2.3). The resulting Gordon identities can raise the photon momentum powers so that the definition of $n$ is no longer meaningful. Scalar Compton scattering is an exception because the first five tensors do not involve $\gamma$-matrices and can be pulled out from $\Lambda_{+}\left(p_{f}\right) \ldots \Lambda_{+}\left(p_{i}\right)$.

In any case, the $X_{i}$ basis in Table I is minimal because no division is necessary in its derivation (see Appendix B). This is signalled by the fact that all CFFs in Tables IV, VI and IX below are free of kinematic singularities and no kinematic factors appear in their denominators. Any basis transformation whose determinant is a constant preserves this property, i.e.,

$$
X_{i}^{\prime}=U_{i j} X_{j}, \quad \operatorname{det} U=\text { const. }
$$

because otherwise the transformation would become singular at specific kinematic points. The standard example of a minimal basis is Tarrach's (modified) basis [29] which is given in Table XVII.

We constructed the $X_{i}$ in Table I to facilitate the physical interpretation:

(i) $X_{1}$ and $X_{2}$ are the Compton tensors that survive for a pointlike scalar particle (cf. Sec. III);

(ii) $X_{1}$ and $X_{10}$ are the tensors for a pointlike fermion, such as the electron in tree-level QED (see Table IV and Sec. IV C);

(iii) $X_{2}$ and $X_{3}$ are the tensors for a scalar $t$-channel exchange, i.e., the CFFs $c_{2}$ and $c_{3}$ have scalar poles (cf. Sec. II in Ref. [50]);

(iv) $X_{6}$ is the tensor for pseudoscalar $t$-channel exchange and therefore $c_{6}$ contains the pion pole. 


\section{Kinematic limits}

We conclude this section with a discussion of the various kinematic limits. As is well known $[1,29,30]$, the 18 CFFs in general kinematics collapse into four CFFs in the forward limit, six CFFs in RCS and 12 CFFs in VCS. With the notation in Table I and Eq. (2.26) these properties are comparatively easy to derive.

In the RCS limit both photons are real $\left(\eta_{+}=\omega=0\right)$. In that case all instances of $t_{Q^{\prime} Q^{\prime}}^{\mu \alpha}$ and $t_{Q Q}^{\alpha \nu}$, which up to factors $Q^{\prime 2}$ and $Q^{2}$ are the transverse projectors, vanish after contraction with the transverse polarization vectors:

$$
\begin{gathered}
\varepsilon^{* \mu}\left(Q^{\prime}\right) t_{Q^{\prime} Q^{\prime}}^{\mu \alpha}=Q^{\prime 2} \varepsilon^{* \alpha}\left(Q^{\prime}\right) \stackrel{Q^{\prime 2}=0}{\rightarrow} 0, \\
t_{Q Q^{\alpha \nu}}^{\alpha \nu}(Q)=Q^{2} \varepsilon^{\alpha}(Q) \stackrel{Q^{2}=0}{\rightarrow} 0 .
\end{gathered}
$$

For example, one can see from Eq. (2.26) that the tensors $X_{3}, X_{4}, X_{5}, X_{7}$ and $X_{8}$ vanish in RCS. In total only six tensors are non-zero, namely $X_{1}, X_{2}, X_{6}, X_{10}, X_{11}$ and $X_{12}$, and thus the RCS amplitude is described by the corresponding six CFFs which depend on $\eta_{-}$and $\lambda^{2}$. Their relations with the RCS amplitudes $A_{i}$ defined in Refs. [51,52] can be found in Table XVIII in Appendix B. In the limit $\eta_{-} \rightarrow 0$ and $\lambda \rightarrow 0$ they are related with the nucleon's static polarizabilities: the electric and magnetic polarizabilities $\alpha$ and $\beta$,

$$
\left[\begin{array}{c}
\alpha+\beta \\
\beta
\end{array}\right]=-\frac{\alpha_{\mathrm{em}}}{m^{3}}\left[\begin{array}{l}
c_{1} \\
c_{2}
\end{array}\right]
$$

and the four spin polarizabilities

$$
\left[\begin{array}{c}
\gamma_{E 1 E 1} \\
\gamma_{M 1 M 1} \\
\gamma_{E 1 M 2} \\
\gamma_{M 1 E 2}
\end{array}\right]=\frac{\alpha_{\mathrm{em}}}{2 m^{4}}\left[\begin{array}{c}
c_{6}+4 c_{11}-4 c_{12} \\
-c_{6}-2 c_{10}+4 c_{12} \\
c_{6}+2 c_{10} \\
-c_{6}
\end{array}\right] .
$$

The forward polarizability $\gamma_{0}$ and so-called pion polarizability $\gamma_{\pi}$ are their linear combinations

$$
\left[\begin{array}{l}
\gamma_{0} \\
\gamma_{\pi}
\end{array}\right]=-\frac{2 \alpha_{\mathrm{em}}}{m^{4}}\left[\begin{array}{c}
c_{11} \\
c_{6}+c_{10}+c_{11}-2 c_{12}
\end{array}\right]
$$

The magnitudes of the CFFs in this limit can be reconstructed from the experimental results for the polarizabilities as well as from ChPT and dispersion relations (see e.g., Table 8 in Ref. [1] and Table 4.2 in [5] for compilations). For example, the $\mathcal{O}\left(p^{3}\right)$ heavy-baryon ChPT calculations for the polarizabilities yield $[53,54]$

$$
\begin{aligned}
& {\left[\begin{array}{l}
c_{1} \\
c_{2}
\end{array}\right]=-\mathcal{C} \frac{\pi m g_{A}}{4 m_{\pi}}\left[\begin{array}{c}
11 \\
1
\end{array}\right],} \\
& {\left[\begin{array}{c}
c_{6} \\
c_{10} \\
c_{11} \\
c_{12}
\end{array}\right]=\mathcal{C} \frac{m^{2}}{m_{\pi}^{2}}\left[\begin{array}{c}
12-g_{A} \\
g_{A} \\
-g_{A} \\
0
\end{array}\right],}
\end{aligned}
$$

where the first term in $c_{6}$ is due to the $t$-channel pion pole. Here, $m_{\pi}$ and $f_{\pi}$ are the pion mass and decay constant, $g_{A}$ is the nucleon's axial charge and we abbreviated

$$
\mathcal{C}=\frac{g_{A}}{3}\left(\frac{m}{4 \pi f_{\pi}}\right)^{2}
$$

Note that the CFFs diverge with powers of $1 / m_{\pi}$ in the chiral limit.

In the VCS limit $\left(\eta_{+}=\omega\right)$ one has $Q^{\prime 2}=0$ and thus only the outgoing photon is real. Only instances of $t_{Q^{\prime} Q^{\prime}}^{\mu \alpha}$ vanish upon contracting with polarization vectors, such as $X_{3}$ and $X_{4}$ in Eq. (2.26), whereas others such as $X_{7}$ and $X_{8}$ become linearly dependent. One arrives at six relations

$$
X_{3}=X_{4}=X_{13}=0, \quad \begin{array}{r}
X_{8}=-\eta_{+} X_{7}, \\
X_{16}=\eta_{+} X_{15}, \\
X_{18}=-\eta_{+} X_{17}
\end{array}
$$

which leaves 12 independent CFFs in VCS:

$$
\begin{aligned}
& c_{1}, \quad c_{6}, \quad c_{10}, \quad c_{14}, \\
& c_{2}, \quad c_{7}-\eta_{+} c_{8}, \quad c_{11}, \quad c_{15}+\eta_{+} c_{16}, \\
& c_{5}, \quad c_{9}, \quad c_{12}, \quad c_{17}-\eta_{+} c_{18} .
\end{aligned}
$$

They are functions of $\eta_{+}, \eta_{-}$and $\lambda^{2}$. In the limit $\eta_{-} \rightarrow 0$ and $\lambda \rightarrow 0$ they are related to the nucleon's generalized polarizabilities [1,6-9], which can be reconstructed with the help of Table XIX in the Appendix.

In the doubly-virtual forward limit, which is defined by $\eta_{+}=\eta_{-}=Q^{2} / m^{2}$ and $\omega=0$, both photons are virtual but because of $Q^{\mu}=Q^{\prime \mu}$ many basis tensors vanish or become linearly dependent. In the simpler cases this can be read off directly from Eq. (2.26), e.g.,

$$
\begin{aligned}
& X_{3}^{\mu \nu}=\frac{1}{m^{4}} t_{Q Q}^{\mu \alpha} t_{Q Q}^{\alpha \nu}=\frac{Q^{2}}{m^{4}} t_{Q Q}^{\mu \nu}=\eta_{+} X_{2}^{\mu \nu}, \\
& X_{6}^{\mu \nu}=\frac{1}{m^{2}} \varepsilon_{Q Q}^{\mu \nu}=0,
\end{aligned}
$$

etc. Note also that

$$
X_{7}^{\mu \nu}=\frac{1}{i m^{3}}\left(Q^{2} \varepsilon_{\gamma Q}^{\mu \nu}-Q^{2} \varepsilon_{Q \gamma}^{\mu \nu}\right)=\frac{2 \eta_{+}}{m} i \varepsilon_{Q \gamma}^{\mu \nu},
$$


so we can drop the factor $\eta_{+}$and thereby define a new tensor with a lower power $n=1$. In total only four independent tensors survive in the forward limit:

$$
\begin{array}{ll}
X_{1}^{\mu \nu}=\frac{1}{m^{4}} Q_{Q p}^{\mu \alpha} t_{p Q}^{\alpha \nu}, & X_{2}^{\mu \nu}=\frac{1}{m^{2}} t_{Q Q}^{\mu \nu}, \\
\tilde{X}_{7}^{\mu \nu}=\frac{1}{m} i \varepsilon_{Q \gamma}^{\mu \nu}, & X_{12}^{\mu \nu}=\frac{\lambda}{m^{2}}\left[t_{Q \gamma}^{\mu \alpha}, t_{\gamma Q}^{\alpha \nu}\right],
\end{array}
$$

whereas $X_{6}=X_{8}=X_{9}=X_{14}=X_{16}=X_{18}=0$ and the remaining ones can be related to them:

$$
\begin{array}{ll}
X_{3}=\eta_{+} X_{2}, & X_{11}=2 \lambda^{2} \tilde{X}_{7}+\frac{1}{4} \eta_{+} X_{12} \\
X_{4}=\eta_{+} X_{1}-\lambda^{2} X_{2}, & X_{13}=\eta_{+} X_{12}, \\
X_{5}=-2 \lambda^{2} X_{2}, & X_{15}=-2 \eta_{+} \tilde{X}_{7}-X_{12}, \\
X_{10}=-\eta_{+} \tilde{X}_{7}, & X_{17}=2 \lambda^{2} X_{2} .
\end{array}
$$

The forward Compton amplitude-more precisely, the bracket in Eq. (2.3) - then becomes

$$
[\ldots]=\bar{c}_{1} X_{1}+\bar{c}_{2} X_{2}+\bar{c}_{3} \tilde{X}_{7}+\bar{c}_{4} X_{12}
$$

where the four CFFs depend on $\eta_{+}=\eta_{-}$and $\lambda^{2}$ :

$$
\begin{aligned}
& \bar{c}_{1}=c_{1}+\eta_{+} c_{4}, \\
& \bar{c}_{2}=c_{2}+\eta_{+} c_{3}-\lambda^{2}\left(c_{4}+2 c_{5}-2 c_{17}\right), \\
& \bar{c}_{3}=\eta_{+}\left(2 c_{7}-c_{10}-2 c_{15}\right)+2 \lambda^{2} c_{11}, \\
& \bar{c}_{4}=c_{12}-c_{15}+\eta_{+}\left(c_{13}+\frac{1}{4} c_{11}\right) .
\end{aligned}
$$

Their relations to the forward amplitudes $T_{1,2}$ and $S_{1,2}$ defined as in [5], whose imaginary parts are proportional to the nucleon structure functions, are given by

$$
\left[\begin{array}{c}
T_{1} \\
T_{2} \\
S_{1} \\
S_{2}
\end{array}\right]=-\frac{4 \pi \alpha_{\mathrm{em}}}{m}\left[\begin{array}{c}
\lambda^{2} \bar{c}_{1}+\eta_{+} \bar{c}_{2} \\
\eta_{+} \bar{c}_{1} \\
\bar{c}_{3} \\
-2 \lambda \bar{c}_{4}
\end{array}\right] .
$$

From their expansion around $\eta_{ \pm}=\lambda=\omega=0$ one can extract several further relations such as the one for the longitudinal-transverse polarizability $\delta_{L T}$ :

$$
\delta_{L T}=-\frac{2 \alpha_{\mathrm{em}}}{m^{4}}\left(c_{11}-c_{12}+c_{15}\right) .
$$

Another example is the doubly-virtual but off-forward VVCS limit, where $Q^{2}=Q^{\prime 2}$ and therefore $\omega=0$ but $\eta_{+} \neq \eta_{-}$. Also here the tensor basis becomes redundant, however in a way where kinematic singularities cannot be avoided. The characteristics already appear in scalar Compton scattering defined by the tensors $X_{1 \ldots 5}$, cf. Refs. $[18,29,33,34]$. In terms of the $K_{i}$ from Table XV one can see that $K_{5}=K_{8}=K_{10}=0$ for $\omega=0$. At first sight this does not seem to affect the $X_{i}$ because Table XVI still implies

$$
\begin{aligned}
& X_{1}=\lambda^{2} K_{1}+\eta_{-} K_{6}+K_{7}, \\
& X_{2}=\eta_{-} K_{1}-K_{3}, \\
& X_{3}=\eta_{+}^{2} K_{1}+\eta_{-} K_{2}-\eta_{+} K_{4}, \\
& X_{4}=\lambda^{2} K_{2}+\eta_{+}^{2} K_{6}+\eta_{+} K_{9}, \\
& X_{5}=-\lambda^{2}\left(2 \eta_{+} K_{1}-K_{4}\right)-\eta_{+} K_{7}+\eta_{-} K_{9} .
\end{aligned}
$$

However, the combination ${ }^{2}$

$$
\eta_{+}^{2} X_{1}+\lambda^{2} X_{3}-\eta_{-} X_{4}+\eta_{+} X_{5}=0
$$

vanishes, as one can verify, and thus one has a nontrivial relation between the $X_{i}$ which cannot be solved without introducing kinematic singularities. In the limit $\omega=0$ one then needs a redundant basis to avoid them. In general kinematics there is no problem: the $18 \mathrm{CFFs}$ and corresponding tensors are regular in the limit $\omega \rightarrow 0$; only when they collapse into fewer independent functions those functions can acquire kinematic singularities.

\section{SCALAR COMPTON AMPLITUDE}

To illustrate the procedure of working out the resonance contributions, we start with the tree-level Compton amplitude for a pointlike scalar particle as a template; see also Refs. [33,34,55]. With the momentum definitions in Eq. (2.7) the Born terms in Fig. 1 read

$$
\begin{aligned}
\Gamma_{\mathrm{B}}^{\mu \nu}\left(p, Q, Q^{\prime}\right)= & \bar{\Gamma}^{\mu}\left(p_{i}^{+}, Q^{\prime}\right) D(p+\Sigma) \Gamma^{\nu}\left(p_{f}^{+}, Q\right) \\
& +\bar{\Gamma}^{\nu}\left(p_{f}^{-},-Q\right) D(p-\Sigma) \Gamma^{\mu}\left(p_{i}^{-},-Q^{\prime}\right) .
\end{aligned}
$$

The scalar propagators depend on the $s$ - and $u$-channel momenta $p \pm \Sigma$; at tree level they are given by

$$
D(p \pm \Sigma)=\frac{1}{(p \pm \Sigma)^{2}+m^{2}}=\frac{1}{m^{2}} \frac{1}{\eta_{-} \mp 2 \lambda} .
$$

The arguments of the scalar-photon vertices are the photon momenta $Q, Q^{\prime}$ and the average momenta of the scalar particle:

$$
p_{f}^{ \pm}=p \pm \frac{Q^{\prime}}{2}, \quad p_{i}^{ \pm}=p \pm \frac{Q}{2} .
$$

The tree-level vertex is $\Gamma^{\mu}(k, Q)=2 k^{\mu}$ and its charge conjugate is defined as $\bar{\Gamma}^{\mu}(k, Q)=\Gamma^{\mu}(-k,-Q)=-2 k^{\mu}$.

\footnotetext{
${ }^{2}$ This is the tensor $\lambda \omega \tau_{5} / m^{4}$ in Tarrach's basis, cf. Table XVII, which vanishes for $\omega=0$.
} 
The Born contribution thus becomes

$$
\begin{aligned}
\Gamma_{\mathrm{B}}^{\mu \nu} & =-\frac{4}{m^{2}}\left[\frac{p_{i}^{+\mu} p_{f}^{+\nu}}{\eta_{-}-2 \lambda}+\frac{p_{i}^{-\mu} p_{f}^{-\nu}}{\eta_{-}+2 \lambda}\right] \\
& =-\frac{8}{\eta_{-}^{2}-4 \lambda^{2}}\left[\eta_{-}\left(\frac{K_{3}^{\mu \nu}}{4}+K_{6}^{\mu \nu}\right)+K_{7}^{\mu \nu}\right],
\end{aligned}
$$

where the $K_{i}^{\mu \nu}$ are the tensors from Table XV:

$$
\begin{aligned}
& K_{3}^{\mu \nu}=\frac{Q^{\mu} Q^{\prime \nu}}{m^{2}}, \quad K_{6}^{\mu \nu}=\frac{p^{\mu} p^{\nu}}{m^{2}}, \\
& K_{7}^{\mu \nu}=\frac{\lambda}{m^{2}}\left(p^{\mu} Q^{\prime \nu}+Q^{\mu} p^{\nu}\right) .
\end{aligned}
$$

Note that the $s$ and $u$-channel poles at $\eta_{-}= \pm 2 \lambda$ enter the denominator in combination and thereby ensure crossing symmetry.

Comparing this with Table XVI, we can recast the result in terms of the transverse tensors $X_{i}^{\mu \nu}$ from Table I,

$$
\Gamma_{\mathrm{B}}^{\mu \nu}=\frac{1}{\eta_{-}^{2}-4 \lambda^{2}}\left(\tilde{c}_{1} X_{1}^{\mu \nu}+\tilde{c}_{2} X_{2}^{\mu \nu}\right)-2 K_{1}^{\mu \nu}
$$

and read off the resulting CFF residues:

$$
\tilde{c}_{1}=-8, \quad \tilde{c}_{2}=2 \eta_{-} .
$$

The Born term is not gauge invariant due to the remainder proportional to $K_{1}^{\mu \nu}=\delta^{\mu \nu}$, but this is only so because the scalar theory has a pointlike seagull interaction similar to the rightmost diagram in Fig. 1:

$$
\Gamma_{1 \mathrm{PI}}^{\mu \nu}\left(p, Q, Q^{\prime}\right)=2 \delta^{\mu \nu}
$$

Adding it cancels the gauge part and ensures that the full Compton amplitude $\Gamma_{\mathrm{B}}^{\mu \nu}+\Gamma_{1 \mathrm{PI}}^{\mu \nu}$ is transverse. As a result, it is completely specified by $\tilde{c}_{1}$ and $\tilde{c}_{2}$.

One could generalize the discussion by calculating corrections to the propagator, the vertex, and the 1PI structure part, e.g., in an effective field theory. As long as the theory respects electromagnetic gauge invariance, the resulting Compton amplitude is fully transverse. The most general form of the offshell vertex allowed by gauge invariance, which is free of kinematic singularities, is

$$
\Gamma^{\mu}(k, Q)=2 f_{1} k^{\mu}+f_{2} t_{Q Q}^{\mu \nu} k^{\nu} .
$$

$t_{Q Q}^{\mu \nu}$ is defined in (2.25) and $f_{1}, f_{2}$ are functions of $k^{2}$, $w=k \cdot Q$ and $Q^{2}$. The form factor $f_{1}$ is determined by the Ward-Takahashi identity (WTI)

$$
\begin{aligned}
Q^{\mu} \Gamma^{\mu}(k, Q) & =D\left(k_{+}\right)^{-1}-D\left(k_{-}\right)^{-1} \\
\Rightarrow f_{1} & =\frac{D\left(k_{+}\right)^{-1}-D\left(k_{-}\right)^{-1}}{k_{+}^{2}-k_{-}^{2}},
\end{aligned}
$$

with $k_{ \pm}=k \pm Q / 2$ and thus $k_{+}^{2}-k_{-}^{2}=2 k \cdot Q$, so that only $f_{2}$ carries dynamical information.

The recipe for deriving Eq. (3.8) is the same as for the more complicated cases in the following sections, such as the nucleon-photon vertex in Sec. IV, the nucleon-toresonance transition vertices in Secs. V and VI, and finally the Compton amplitude in Appendix B. We start with the general decomposition

$$
\Gamma^{\mu}(k, Q)=a_{1} k^{\mu}+a_{2} w Q^{\mu},
$$

where $w=k \cdot Q$ ensures the correct charge-conjugation parity: $\bar{\Gamma}^{\mu}(k, Q)=-\Gamma^{\mu}(k,-Q)$. As a consequence, $a_{1}$ and $a_{2}$ are even in $w$ and only depend on $w^{2}$. Next, we derive the transverse part of the vertex by solving

$$
Q^{\mu} \Gamma^{\mu}(k, Q)=w\left(a_{1}+a_{2} Q^{2}\right)=0 .
$$

This must be done without introducing kinematic singularities, i.e., we must solve for $a_{1}$ (and not $a_{2}$ ) which leads to the transverse part $\propto t_{Q Q}^{\mu \nu} k^{\nu}$. Relaxing again the transversality constraint, we then add the term $\propto k^{\mu}$ that we eliminated (and not $Q^{\mu}$ ), which constitutes the gauge part and leads to the result (3.8). Finally, solving the WTI in Eq. (3.9) determines the coefficient $f_{1}$.

The same procedure is carried out in Appendix B to derive the tensor basis for the Compton amplitude itself, although in that case the gauge parts must vanish because the amplitude is transverse. In general neither the Born terms nor the structure part alone are gauge invariant, but one can project them onto a complete basis where the sum of the gauge parts must cancel in the end like in the simple case (3.5)-(3.7).

In the following we are interested in the nucleon Born terms and nucleon resonance contributions to Compton scattering. In those cases one can enforce gauge invariance from the beginning by imposing appropriate constraints on the vertices (which is also possible because there is no seagull term for fermions).

\section{NUCLEON BORN TERM}

Returning to nucleon Compton scattering, the Born term for the nucleon has the form

$$
\begin{aligned}
\Gamma_{\mathrm{B}}^{\mu \nu}= & \Lambda_{+}\left(p_{f}\right)\left[\bar{\Gamma}^{\mu}\left(p_{i}^{+}, Q^{\prime}\right) S(p+\Sigma) \Gamma^{\nu}\left(p_{f}^{+}, Q\right)\right. \\
& \left.+\bar{\Gamma}^{\nu}\left(p_{f}^{-},-Q\right) S(p-\Sigma) \Gamma^{\mu}\left(p_{i}^{-},-Q^{\prime}\right)\right] \Lambda_{+}\left(p_{i}\right) .
\end{aligned}
$$

Here, $\Gamma^{\mu}(k, Q)$ is the dressed offshell nucleon-photon vertex that depends on the average nucleon momentum 
$k$ and the total photon momentum $Q \cdot p_{f}^{ \pm}$and $p_{i}^{ \pm}$were defined in (3.2). The charge-conjugate vertex is

$$
\bar{\Gamma}^{\mu}(k, Q)=C \Gamma^{\mu}(-k,-Q)^{T} C^{T},
$$

where $C=\gamma^{4} \gamma^{2}$ is the charge-conjugation matrix that satisfies $C^{T}=C^{-1}=-C$. The charge-conjugation symmetry of the nucleon-photon vertex amounts to

$$
\bar{\Gamma}^{\mu}(k, Q)=-\Gamma^{\mu}(k,-Q) .
$$

The Born term (4.1) shares the symmetries of the full Compton amplitude, namely Bose (photon-crossing) and charge-conjugation invariance as in Eq. (2.19).

The nucleon propagator and its inverse are given by

$$
\begin{aligned}
S(k) & =\frac{1}{A\left(k^{2}\right)} \frac{-i \not k+M\left(k^{2}\right)}{k^{2}+M\left(k^{2}\right)^{2}}, \\
S(k)^{-1} & =A\left(k^{2}\right)\left(i \not k+M\left(k^{2}\right)\right),
\end{aligned}
$$

where $M\left(k^{2}\right)$ and $A\left(k^{2}\right)$ are momentum-dependent functions. In practice we treat the nucleon as a constituent-like particle and set $M\left(k^{2}\right)=m$ and $A\left(k^{2}\right)=1$, which holds on the mass shell $k^{2}=-m^{2}$, but to keep the discussion general we will retain the momentum dependence in the following two subsections.

\section{A. Offshell nucleon-photon vertex}

First we derive the general form of the offshell nucleonphoton vertex. The discussion is based on the quark-photon vertex [27,56,57] but it can be equally applied to nucleon resonances. The kinematics are sketched in Fig. $4 ; k_{ \pm}=$ $k \pm Q / 2$ are the incoming and outgoing nucleon momenta. Electromagnetic gauge invariance leads to a WardTakahashi identity (WTI) for the vertex,

$$
Q^{\mu} \Gamma^{\mu}(k, Q)=\mathcal{Z}\left[S\left(k_{+}\right)^{-1}-S\left(k_{-}\right)^{-1}\right],
$$

with $\mathcal{Z}=1(\mathcal{Z}=0)$ for the proton (neutron). It can thus be written as the sum of a "gauge part" and a transverse part, where the former is constrained by the WTI:

$$
\Gamma^{\mu}(k, Q)=\Gamma_{\mathrm{G}}^{\mu}(k, Q)+\Gamma_{\perp}^{\mu}(k, Q) .
$$

In the case of a nucleon resonance the r.h.s. of Eq. (4.5) is zero and the vertex is purely transverse.

To derive both contributions, we start from the general offshell fermion-photon vertex for a spin- $1 / 2$ particle:

$$
\Gamma^{\mu}(k, Q)=\sum_{n=1}^{12} h_{n}\left(k^{2}, w, Q^{2}\right) i \tau_{n}^{\mu}(k, Q) .
$$

The $h_{i}$ are Lorentz-invariant functions, with $w=k \cdot Q$, and the $\tau_{i}^{\mu}$ are the 12 possible tensors permitted by Lorentz covariance and parity invariance:

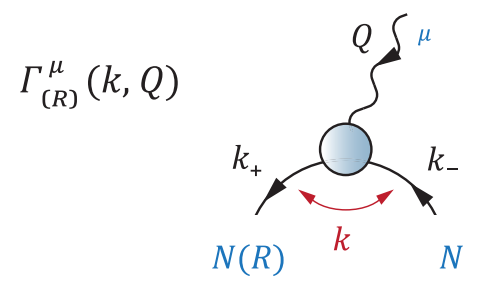

FIG. 4. Kinematics in the nucleon-photon and $N \rightarrow \frac{1}{2}^{ \pm}$transition vertex.

$$
\begin{aligned}
& \gamma^{\mu} \quad i k^{\mu} \quad i w Q^{u} \\
& i w\left[\gamma^{\mu}, \not\right] \quad k^{\mu} \not k \quad w Q^{\mu} \not k
\end{aligned}
$$

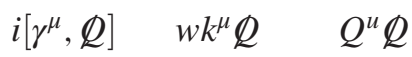

$$
\begin{aligned}
& {\left[\gamma^{\mu}, \not k, Q\right] \quad i k^{\mu}[\not k, Q] \quad i w Q^{\mu}[\not k, Q] \text {. }}
\end{aligned}
$$

We took commutators and attached factors of $w$ to ensure that they all share the charge-conjugation symmetry (4.3) of the full vertex, so that the $h_{i}$ are even in $w$ and only depend on $w^{2}$. We label the tensors column-wise: $\tau_{1 \ldots 4}^{\mu}, \tau_{5 \ldots 8}^{\mu}$ and $\tau_{9 \ldots 12}^{\mu}$ are the elements in the first, second and third column, respectively.

To derive the transverse part of the vertex we work out the condition $Q^{\mu} \Gamma^{\mu}=0$. The contraction produces four independent tensors $\sim \mathbb{1}, \mathscr{Q}, \not k,[K, Q]$ and thus four relations between the dressing functions, which must be solved so that no kinematic singularities are introduced in the process. The result

$$
\begin{array}{ll}
h_{1}=-w^{2} h_{7}-Q^{2} h_{11}, & h_{5}=-Q^{2} h_{9}, \\
h_{2}=h_{8}+Q^{2} h_{12}, & h_{6}=-Q^{2} h_{10}
\end{array}
$$

is almost unique: without dividing by factors of $Q^{2}$ or $w^{2}$, our only freedom is to solve for either $h_{2}$ or $h_{8}$. Substitution into (4.7) yields the transverse vertex

$$
\Gamma_{\perp}^{\mu}(k, Q)=\sum_{n=1}^{8} f_{n}\left(k^{2}, w, Q^{2}\right) i T_{n}^{\mu}(k, Q)
$$

where the $f_{n}$ are the remaining independent functions and $T_{n}^{\mu}$ the transverse tensors in Table II. This defines a minimal basis where transversality and analyticity are manifest: the $T_{n}^{\mu}$ are transverse and regular for $Q^{\mu} \rightarrow 0$ and the $f_{n}$ are free of kinematic singularities at $Q^{2} \rightarrow 0$ and kinematically independent.

The remaining gauge part in (4.6) can only depend on the tensors for $h_{1}, h_{2}, h_{5}$, and $h_{6}$ that we eliminated under the assumption that the vertex was transverse; these are the $G_{i}^{\mu}$ in Table II. Putting them back into the WTI (4.5) together with the nucleon propagator (4.4) determines their coefficients and leads to the Ball-Chiu vertex [58]:

$$
\Gamma_{\mathrm{G}}^{\mu}(k, Q)=i \mathcal{Z}\left[\Sigma_{A} G_{1}^{\mu}+2 m^{2} \Delta_{A} G_{2}^{\mu}-2 m \Delta_{B} G_{3}^{\mu}\right] .
$$


TABLE II. Top: Eight tensors $T_{i}^{\mu}$ constituting the transverse part $\Gamma_{\perp}^{\mu}(k, Q)$ of the offshell nucleon-photon vertex without introducing kinematic singularities. $t_{A B}^{\mu \nu}$ and the triple commutator are defined in Eqs. (2.25) and (A15). Bottom: Four tensors $G_{i}^{\mu}$ of the gauge part $\Gamma_{\mathrm{G}}^{\mu}(k, Q)$. We attached powers of the nucleon mass $m$ to make all tensors dimensionless.

\begin{tabular}{ll}
\hline \hline$m^{2} T_{1}^{\mu}=t_{Q Q}^{\mu \nu} \gamma^{\nu}$ & $m^{3} T_{5}^{\mu}=t_{Q Q}^{\mu \nu} i k^{\nu}$ \\
$m^{5} T_{2}^{\mu}=t_{Q Q}^{\mu \nu} w \frac{i}{2}\left[\gamma^{\nu}, \not k\right.$ & $m^{4} T_{6}^{\mu}=t_{Q Q}^{\mu \nu} k^{\nu} \not k$ \\
$m T_{3}^{\mu}=\frac{i}{2}\left[\gamma^{\mu}, Q\right]$ & $m^{4} T_{7}^{\mu}=t_{Q k}^{\mu \nu} w \gamma^{\nu}$ \\
$m^{2} T_{4}^{\mu}=\frac{1}{6}\left[\gamma^{\mu}, \not k, Q\right]$ & $m^{3} T_{8}^{\mu}=t_{Q k}^{\mu \nu} \frac{i}{2}\left[\gamma^{\nu}, \not k\right]$ \\
$G_{1}^{\mu}=\gamma^{\mu}$ & $m G_{3}^{\mu}=i k^{\mu}$ \\
$m^{2} G_{2}^{\mu}=k^{\mu} \not k$ & $m^{3} G_{4}^{\mu}=w \frac{i}{2}\left[\gamma^{\mu}, \not l\right]$ \\
\hline \hline
\end{tabular}

It is fully specified by the nucleon propagator and depends on sums and difference quotients of the propagator dressing functions $A\left(k^{2}\right)$ and $M\left(k^{2}\right)$ :

$$
\Sigma_{F}=\frac{F\left(k_{+}^{2}\right)+F\left(k_{-}^{2}\right)}{2}, \quad \Delta_{F}=\frac{F\left(k_{+}^{2}\right)-F\left(k_{-}^{2}\right)}{k_{+}^{2}-k_{-}^{2}},
$$

where $F \in\{A, B\}$ and $B\left(k^{2}\right)=A\left(k^{2}\right) M\left(k^{2}\right)$. Note that $G_{4}^{\mu}$ drops out as a consequence of electromagnetic gauge invariance. For a tree-level nucleon propagator the gauge part reduces to $\Gamma_{\mathrm{G}}^{\mu}(k, Q)=\mathcal{Z} i \gamma^{\mu}$.

We should emphasize that the gauge part is not longitudinal. One could equally split the vertex into longitudinal and transverse parts, where the longitudinal tensors are proportional to $Q^{\mu}$ and defined by the rightmost column in (4.8). In that case the WTI would still only affect the longitudinal part, but because the transverse projector has a kinematic singularity at $Q^{2}=0$ the longitudinal and transverse dressing functions would become kinematically related at the origin and/or show kinematic zeros. Thus, in analogy to Eq. (3.8) for the scalar vertex, only the separation into $\Gamma_{\mathrm{G}}^{\mu}$ and $\Gamma_{\perp}^{\mu}$ ensures that the resulting dressing functions are truly kinematically independent.

\section{B. On-shell nucleon-photon current}

The onshell current follows from sandwiching the vertex between nucleon spinors (or positive-energy projectors) and taking the nucleon momenta onshell:

$$
J^{\mu}(k, Q)=\left.\Lambda_{+}\left(k_{+}\right) \Gamma^{\mu}(k, Q) \Lambda_{+}\left(k_{-}\right)\right|_{\text {onshell }} \cdot
$$

The limit $k_{+}^{2}=k_{-}^{2}=-m^{2}$ entails $k^{2}=-m^{2}-Q^{2} / 4$ and $w=0$, so the only remaining independent variable is $Q^{2}$. The 12 offshell tensors collapse into two, $G_{1}^{\mu}$ and $T_{3}^{\mu}$, by means of the Gordon identities in Table III. The current takes the standard Dirac form:

$$
J^{\mu}(k, Q)=i \Lambda_{+}\left(k_{+}\right)\left[F_{1} \gamma^{\mu}+\frac{i F_{2}}{4 m}\left[\gamma^{\mu}, Q\right]\right] \Lambda_{+}\left(k_{-}\right),
$$

TABLE III. Combinations of tensors that vanish in the on-shell projection (4.12). We abbreviated $\tau=Q^{2} /\left(4 m^{2}\right)$.

\begin{tabular}{ll}
\hline \hline$T_{1}-4 \tau G_{1}$ & $T_{7}$ \\
$T_{2}$ & $T_{8}-2 \tau\left(G_{1}-\frac{1}{2} T_{3}\right)$ \\
$T_{4}+2 \tau G_{1}+T_{3}$ & $G_{2}+G_{1}-\frac{1}{2} T_{3}$ \\
$T_{5}+4 \tau\left(G_{1}-\frac{1}{2} T_{3}\right)$ & $G_{3}+G_{1}-\frac{1}{2} T_{3}$ \\
$T_{6}+4 \tau\left(G_{1}-\frac{1}{2} T_{3}\right)$ & $G_{4}$ \\
\hline \hline
\end{tabular}

where $F_{1}\left(Q^{2}\right)$ and $F_{2}\left(Q^{2}\right)$ are the onshell Dirac and Pauli form factors.

Even though the offshell current has a gauge part, it becomes "accidentally" transverse in the on-shell projection (4.12):

$$
\left.Q^{\mu} \Lambda_{+}\left(k_{+}\right) \gamma^{\mu} \Lambda_{+}\left(k_{-}\right)\right|_{\text {onshell }}=0,
$$

and the same is true for the remaining tensors $G_{2,3,4}^{\mu}$ because $w=k \cdot Q=0$ on the mass shell. It follows from Table III that the gauge part contributes to $F_{1}$ and $F_{2}$ :

$$
\begin{aligned}
& F_{1}\left(Q^{2}\right)=\mathcal{Z}\left[\Sigma_{A}+2 m\left(\Delta_{B}-m \Delta_{A}\right)\right]+\cdots, \\
& F_{2}\left(Q^{2}\right)=-2 m \mathcal{Z}\left(\Delta_{B}-m \Delta_{A}\right)+\cdots,
\end{aligned}
$$

where the dots refer to the transverse pieces. On the mass shell, however, the nucleon propagator is that of a free particle and therefore $\Sigma_{A}=1$ and $\Delta_{A}=\Delta_{B}=0$. As a result, the Dirac and Pauli form factors are related with the off-shell dressing functions via

$$
\begin{aligned}
& F_{1}\left(Q^{2}\right)=\mathcal{Z}+\frac{Q^{2}}{m^{2}}\left[f_{1}-\frac{f_{4}}{2}-\left(f_{5}+f_{6}-\frac{f_{8}}{2}\right)\right], \\
& F_{2}\left(Q^{2}\right)=2\left(f_{3}-f_{4}\right)+\frac{Q^{2}}{m^{2}}\left(f_{5}+f_{6}-\frac{f_{8}}{2}\right),
\end{aligned}
$$

with the $f_{n}$ evaluated at the onshell point.

\section{Compton form factors}

With the off-shell nucleon-photon vertex at hand, we proceed to work out the Compton form factors for the nucleon Born term (4.1). Although the Born term does not contribute to nucleon polarizabilities, it is still relevant for two-photon exchange effects to form factors.

We restrict ourselves to the tree-level propagator

$$
S(k)=\frac{-i \not k+m}{k^{2}+m^{2}},
$$

and instead of the full vertex in Eq. (4.6) we employ the "Dirac form" for the off-shell nucleon-photon vertex:

$$
\Gamma^{\mu}(k, Q)=i\left(F_{1} G_{1}^{\mu}+F_{2} \frac{T_{3}^{\mu}}{2}\right)
$$


With the definition of charge conjugation in Eq. (4.2), the charge-conjugate vertex $\bar{\Gamma}^{\mu}(k, Q)$ differs from the above only by a minus sign in front of the $F_{1}$ term.

While the Dirac and Pauli form factors $F_{i}\left(k^{2}, w, Q^{2}\right)$ in these expressions are offshell, we will identify them with their on-shell expressions $F_{i}\left(Q^{2}\right)$ since this is the only information we can gather from experiments. Employing the Dirac form is also the minimal requirement for keeping the Born term gauge invariant [59]. We would lose transversality if we

(i) equipped $F_{1,2}\left(Q^{2}\right)$ with a $k^{2}$ or $w$ dependence,

(ii) added other tensors $G_{2,3,4}^{\mu}$ from Table II,

(iii) but also other tensors $T_{i}^{\mu}$ (except for $T_{1}^{\mu}$ ) because they lead to interference terms with $G_{1}^{\mu}$ from the second vertex,

(iv) or if we implemented momentum-dependent dressing functions in the nucleon propagator with ramifications for the gauge part of the vertex.

This is all due to the gauge part in the vertex and does not happen for the nucleon resonances which we consider later. It is also not a serious conceptual problem because the twophoton WTI allows one to construct a gauge-invariant completion of the Born term for a general offshell nucleon-photon vertex and nucleon propagator, which can be found in Ref. [27]. A simpler alternative is to project the (nongauge-invariant) Born term onto a full basis and afterwards retain only the transverse part, since all non-transverse pieces must cancel when they are calculated from some consistent underlying theory. We will not further pursue this here and instead provide examples in Appendix B 4.

Inserting the above expressions into Eq. (4.1) yields four mixed terms $F_{i}\left(Q^{\prime 2}\right) F_{j}\left(Q^{2}\right) \equiv F_{i}^{\prime} F_{j}$. We take their symmetric combinations

$$
\begin{array}{ll}
H_{1}=F_{1}^{\prime} F_{1}, & H_{2}=F_{2}^{\prime} F_{2}, \\
H_{3}=\frac{F_{1}^{\prime} F_{2}+F_{2}^{\prime} F_{1}}{2}, & H_{4}=\frac{F_{1}^{\prime} F_{2}-F_{2}^{\prime} F_{1}}{2 \omega},
\end{array}
$$

with $\omega$ defined in (2.6). Note that $H_{4}(\omega \rightarrow 0)$ is regular. The nucleon Born term then takes the form

$$
\Gamma_{\mathrm{B}}^{\mu \nu}=\frac{1}{\eta_{-}^{2}-4 \lambda^{2}} \sum_{i=1}^{18} \tilde{c}_{i}\left[\Lambda_{+}\left(p_{f}\right) X_{i}^{\mu \nu} \Lambda_{+}\left(p_{i}\right)\right],
$$

where the resulting $\mathrm{CFF}$ residues $\tilde{c}_{i}$ are collected in Table IV. For a pointlike fermion $\left(F_{1}=1\right.$ and $\left.F_{2}=0\right)$ only $\tilde{c}_{1}=-4$ and $\tilde{c}_{10}=2$ survive, i.e., $X_{1}^{\mu \nu}$ and $X_{10}^{\mu \nu}$ defined in Table I are the Compton tensors of a structureless fermion such as the electron in tree-level QED.

Because $Q^{2}$ and $Q^{\prime 2}$ are linear combinations of $\eta_{+}$and $\omega$, the $H_{i}$ can only depend on $\eta_{+}$and $\omega^{2}$. In addition, the CFF residues in Table IV depend on the variable $\lambda$ at most quadratically (which is also true for the resonance terms in Tables VI and IX below). It is then customary to rearrange $a-4 \lambda^{2} b=\left(\eta_{-}^{2}-4 \lambda^{2}\right) b+\left(a-\eta_{-}^{2} b\right)$ and split the CFFs into nonresonant and resonant terms:
TABLE IV. Compton form factor residues for the nucleon Born term. The $H_{i}$ are defined in Eq. (4.19). The remaining $\tilde{c}_{i}$ are zero.

$$
\begin{aligned}
& \tilde{c}_{1}=-\left(4 H_{1}+\eta_{-} H_{2}\right) \\
& \tilde{c}_{2}=-\eta_{-}\left(H_{2}+2 H_{3}\right)+\lambda^{2} H_{2} \\
& \tilde{c}_{6}=\eta_{-}\left(H_{3}-\frac{1}{4} \eta_{+} H_{2}\right) \\
& \tilde{c}_{10}=2\left(H_{1}+H_{3}\right)-\frac{1}{2}\left(\eta_{+}-\eta_{-}\right) H_{2} \\
& \tilde{c}_{11}=-H_{2} \\
& \tilde{c}_{12}=-\left(H_{2}+H_{3}\right)-\frac{1}{4}\left(\eta_{+}-\eta_{-}\right) H_{2} \\
& \tilde{c}_{14}=\frac{1}{4} H_{2}+H_{4} \\
& \tilde{c}_{15}=-\frac{1}{4} \eta_{-} H_{2}
\end{aligned}
$$

$$
c_{i}=\frac{\tilde{c}_{i}}{\eta_{-}^{2}-4 \lambda^{2}}=c_{i}^{(0)}+\frac{c_{i}^{(1)}}{\eta_{-}^{2}-4 \lambda^{2}},
$$

where $c_{i}^{(0)}$ and $c_{i}^{(1)}$ no longer depend on $\lambda^{2}$.

In Fig. 5 we plot the $\tilde{c}_{i}$ from Table IV inside the TPE cone shown in Fig. 2, using simple multipole parametrizations for the proton's Dirac and Pauli form factors [60]. The bands show the variation with $\eta_{-}, \omega$ and $\lambda$, which turns out to be almost negligible. Therefore, the dependence on the four variables effectively collapses into a one-dimensional dependence on $\eta_{+}$. This is the typical behavior also for the resonance Born terms in the following sections, which happens in different systems as well $[50,61,62]$ : when implementing Lorentz invariance, permutation-group symmetries and minimal tensor bases, the potentially complicated momentum dependencies of three- and four-point amplitudes often collapse into a simple one-dimensional dependence on the symmetric variable, which in our case is $\eta_{+}$.

In passing we can also verify the low-energy theorem by Low [63], Gell-Mann and Goldberger [64]. In the forward limit where $\eta_{+}=\eta_{-}=Q^{2} / \mathrm{m}^{2}=Q^{\prime 2} / \mathrm{m}^{2}$, the contribution from the nucleon Born term to the forward amplitudes $A_{i}=\left\{T_{1}, T_{2}, 2 S_{1},-S_{2} / \lambda\right\}$ in Eq. (2.42) is
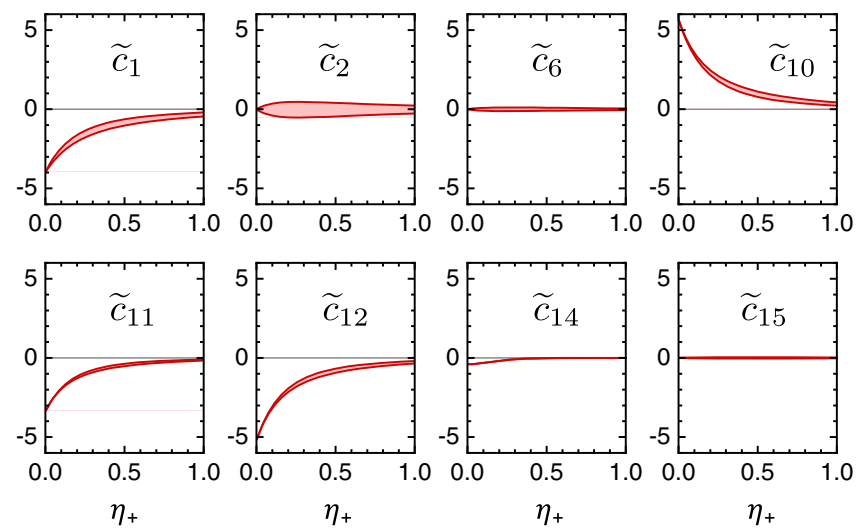

FIG. 5. Compton form factor residues from the nucleon Born term inside the TPE cone and plotted over the variable $\eta_{+}$. 


$$
A_{i}=\frac{4 \pi \alpha_{\mathrm{em}}}{m}\left(-A_{i}^{(0)}+\frac{A_{i}^{(1)}}{\eta_{-}^{2}-4 \lambda^{2}}\right)
$$

where

$$
A_{i}^{(0)}=\left[\begin{array}{c}
F_{1}^{2} \\
0 \\
F_{2}^{2} \\
0
\end{array}\right], \quad A_{i}^{(1)}=\left[\begin{array}{c}
\eta_{-}^{2} G_{M}^{2} \\
\eta_{-}\left(4 F_{1}^{2}+\eta_{-} F_{2}^{2}\right) \\
4 \eta_{-} F_{1} G_{M} \\
2 F_{2} G_{M}
\end{array}\right]
$$

and $G_{M}=F_{1}+F_{2}$ is the proton's magnetic Sachs form factor. In the static limit $\left(\eta_{-}=\lambda=0\right)$ the amplitude $T_{1}$ vanishes according to Eq. (2.42), except when the CFFs are singular. The only singularities in that limit come from the nucleon Born terms, as illustrated in Fig. 3, which produces the Thomson term in $T_{1}$ :

$$
T_{1}-\left.T_{1}^{\text {Pole }}\right|_{\eta_{-}=\lambda=0}=-\frac{4 \pi \alpha_{\mathrm{em}}}{m} \mathcal{Z}^{2} .
$$

\section{SPIN-1/2 RESONANCES}

We proceed with the discussion of $J=1 / 2^{ \pm}$resonances. In Sec. VII A we will explicitly consider the Roper resonance $N(1440)$, the $N(1710)$, the nucleon's parity partner $N(1535)$ and its first excitation $N(1650)$, and the $\Delta(1620)$. However, the following considerations are valid for all $J=1 / 2^{ \pm}$states. In these cases the resonance 'Born terms' conceptually enter in the structure part of Fig. 1. It has the same form as in (4.1),

$$
\begin{aligned}
\Gamma_{1 / 2}^{\mu \nu}= & \Lambda_{+}\left(p_{f}\right)\left[\bar{\Gamma}_{\mathrm{R}}^{\mu}\left(p_{i}^{+}, Q^{\prime}\right) S_{\mathrm{R}}(p+\Sigma) \Gamma_{\mathrm{R}}^{\nu}\left(p_{f}^{+}, Q\right)\right. \\
& \left.+\bar{\Gamma}_{\mathrm{R}}^{\nu}\left(p_{f}^{-},-Q\right) S_{\mathrm{R}}(p-\Sigma) \Gamma_{\mathrm{R}}^{\mu}\left(p_{i}^{-},-Q^{\prime}\right)\right] \Lambda_{+}\left(p_{i}\right),
\end{aligned}
$$

except that $S_{\mathrm{R}}(k)$ is the propagator of the resonance and $\Gamma_{\mathrm{R}}^{\mu}(k, Q)$ the nucleon-to-resonance transition vertex. Equations (3.2) and (4.2) remain valid, but the transition vertex is no longer charge-conjugation invariant because the fermion legs correspond to different particles.

In view of a compact notation we abbreviate

$$
\begin{aligned}
r & =\frac{m_{R}}{m}=\sqrt{1+\delta}, \\
\delta_{ \pm} & =\frac{m_{R} \pm m}{2 m}=\frac{r \pm 1}{2}, \\
\lambda_{ \pm} & =\frac{\left(m_{R} \pm m\right)^{2}+Q^{2}}{4 m^{2}}=\tau+\delta_{ \pm}^{2},
\end{aligned}
$$

with $\delta=4 \delta_{+} \delta_{-}$from Eq. (2.16) and $\tau=Q^{2} /\left(4 m^{2}\right)$.

\section{A. $N \rightarrow \frac{1}{2}^{ \pm}$transition current}

The off-shell transition vertex requires no separate derivation because we only need to drop the gauge part from Eq. (4.6). The WTI simplifies to the transversality condition

$$
Q^{\mu} \Gamma_{\mathrm{R}}^{\mu}(k, Q)=0,
$$

so the vertex is purely transverse and can be expressed through the eight tensors $T_{i}^{\mu}$ in Table II:

$$
\Gamma_{\mathrm{R}}^{\mu}(k, Q)=\left[\begin{array}{c}
\mathbb{1} \\
\gamma_{5}
\end{array}\right] \sum_{n=1}^{8} f_{n}^{\mathrm{R}}\left(k^{2}, w, Q^{2}\right) i T_{n}^{\mu}(k, Q),
$$

where the upper (lower) entry holds for resonances with positive (negative) parity.

The on-shell transition current is analogous to (4.12),

$$
J_{\mathrm{R}}^{\mu}(k, Q)=\left.\Lambda_{+}\left(k_{+}\right) \Gamma_{\mathrm{R}}^{\mu}(k, Q) \Lambda_{+}\left(k_{-}\right)\right|_{\text {onshell }},
$$

except that "on-shell" now refers to the kinematic limit $k_{-}^{2}=-m^{2}, k_{+}^{2}=-m_{R}^{2}$. Therefore,

$$
k^{2}=-m^{2}\left(1+\tau+\frac{\delta}{2}\right), \quad w=-\frac{m^{2}}{2} \delta
$$

and the positive-energy projectors are

$\Lambda_{+}\left(k_{+}\right)=\frac{-i \not k_{+}+m_{R}}{2 m_{R}}, \quad \Lambda_{+}\left(k_{-}\right)=\frac{-i \not k_{-}+m}{2 m}$.

Also in this case the eight tensors collapse into two structures on the mass shell; the corresponding identities are given in Table $\mathrm{V}$ and slightly differ from before. In combination with (5.5) we can write the on-shell current as

$$
\Gamma_{\mathrm{R}}^{\mu}(k, Q)=i\left[\begin{array}{c}
\mathbb{1} \\
\gamma_{5}
\end{array}\right]\left(F_{1} T_{1}^{\mu}+F_{2} \frac{T_{3}^{\mu}}{2}\right)
$$

To avoid clutter we use the same notation for the form factors as before $\left(F_{1}\right.$ and $\left.F_{2}\right)$ but they should not be confused with those of the nucleon.

The notable difference here is the appearance of $T_{1}^{\mu}$ instead of $G_{1}^{\mu}$ because the latter no longer appears in the offshell current. It is usually written as

TABLE V. Combinations of tensors (defined in Table II) for the off-shell nucleon-to-resonance transition vertex that vanish in the on-shell projection (5.5) in the positive-parity case. For negative parity, replace $\delta_{+} \leftrightarrow-\delta_{-}$. The variables $\delta, \delta_{ \pm}$and $\tau$ are defined in (5.2).

\begin{tabular}{ll}
\hline \hline$T_{2}+\frac{1}{2} \delta \delta_{-} T_{1}$ & $T_{6}+\delta_{+}\left(\delta_{+} T_{1}-2 \tau T_{3}\right)$ \\
$T_{4}+\frac{1}{2} T_{1}+\delta_{+} T_{3}$ & $T_{7}-\frac{1}{2} \delta \delta_{-} T_{3}$ \\
$T_{5}+\delta_{+} T_{1}-2 \tau T_{3}$ & $T_{8}-\frac{1}{2}\left(\delta_{+} T_{1}-2 \tau T_{3}\right)+\delta_{-}^{2} T_{3}$ \\
\hline \hline
\end{tabular}




$$
F_{1} T_{1}^{\mu}=F_{1}^{*} \gamma_{\perp}^{\mu} \Rightarrow F_{1}^{*}=\frac{Q^{2}}{m^{2}} F_{1}
$$

where $\gamma_{\perp}^{\mu}=\gamma^{\mu}-Q Q^{\mu} / Q^{2}$ is the transverse projection of the $\gamma$-matrix. This quantity has a kinematic singularity at $Q^{2} \rightarrow 0$, which must be compensated by a kinematic zero in $F_{1}^{*}$. Therefore, $F_{1}^{*}\left(Q^{2} \rightarrow 0\right)=0$ is a consequence of transversality and analyticity and holds for all $J=\frac{1}{2}$ resonance transition form factors alike. This (trivially) exemplifies the advantage of using constraint-free tensor bases: if the current is written in terms of $T_{1}^{\mu}$, the form factor $F_{1}$ approaches a constant and non-zero value for $Q^{2} \rightarrow 0$.

\section{B. $J^{P}=\frac{1}{2} \pm$ resonance Born terms}

The offshell transition vertex does not have a gauge part, so there is also no restriction in the sense of Eq. (4.18) because all eight tensors result in a transverse Born term and there are no gauge parts to interfere with. However, experiment only provides information about on-shell form factors, and therefore we restrict ourselves again to treelevel propagators

$$
S_{\mathrm{R}}(k)=\frac{-i \not k+m_{R}}{k^{2}+m_{R}^{2}}
$$

and form factors $F_{1}\left(Q^{2}\right)$ and $F_{2}\left(Q^{2}\right)$ only:

$$
\begin{aligned}
& \Gamma_{\mathrm{R}}^{\mu}(k, Q)=i\left[\begin{array}{c}
\mathbb{1} \\
\gamma_{5}
\end{array}\right]\left(F_{1} T_{1}^{\mu}+F_{2} \frac{T_{3}^{\mu}}{2}\right), \\
& \bar{\Gamma}_{\mathrm{R}}^{\mu}(k, Q)=i\left(-F_{1} T_{1}^{\mu}+F_{2} \frac{T_{3}^{\mu}}{2}\right)\left[\begin{array}{c}
\mathbb{1} \\
\gamma_{5}
\end{array}\right],
\end{aligned}
$$

where upper (lower) entries correspond to $J^{P}=\frac{1}{2}^{+}\left(\frac{1}{2}^{-}\right)$.

In analogy to (4.19) we employ the symmetric combinations

$$
\Sigma_{i j}=\frac{F_{i}^{\prime} F_{j}+F_{j}^{\prime} F_{i}}{2}, \quad \Delta_{i j}=\frac{F_{i}^{\prime} F_{j}-F_{j}^{\prime} F_{i}}{2 \omega}
$$

but we redefine the $H_{i}$ as

$$
\begin{array}{ll}
H_{1}=\left(\eta_{+}^{2}-\omega^{2}\right) \Sigma_{11}, & H_{2}=\Sigma_{22}, \\
H_{3}=\eta_{+} \Sigma_{12}-\omega^{2} \Delta_{12}, & H_{4}=\eta_{+} \Delta_{12}-\Sigma_{12} .
\end{array}
$$

If we replaced $\left(Q^{2} / m^{2}\right) F_{1} \rightarrow F_{1}$ they would coincide with our earlier definition (4.19) for the nucleon.

The Born term for an intermediate nucleon resonance then becomes

$$
\Gamma_{1 / 2}^{\mu \nu}=\frac{1}{D} \sum_{i=1}^{18} \tilde{c}_{i}\left[\Lambda_{+}\left(p_{f}\right) X_{i}^{\mu \nu} \Lambda_{+}\left(p_{i}\right)\right],
$$

where the resonance pole is given by (cf. Eq. (2.15)
TABLE VI. Compton form factor residues for a $J^{P}=\frac{1}{2}+$ resonance. The $H_{i}$ are defined in Eq. (5.13) and $\delta, \delta_{ \pm}$in (5.2).

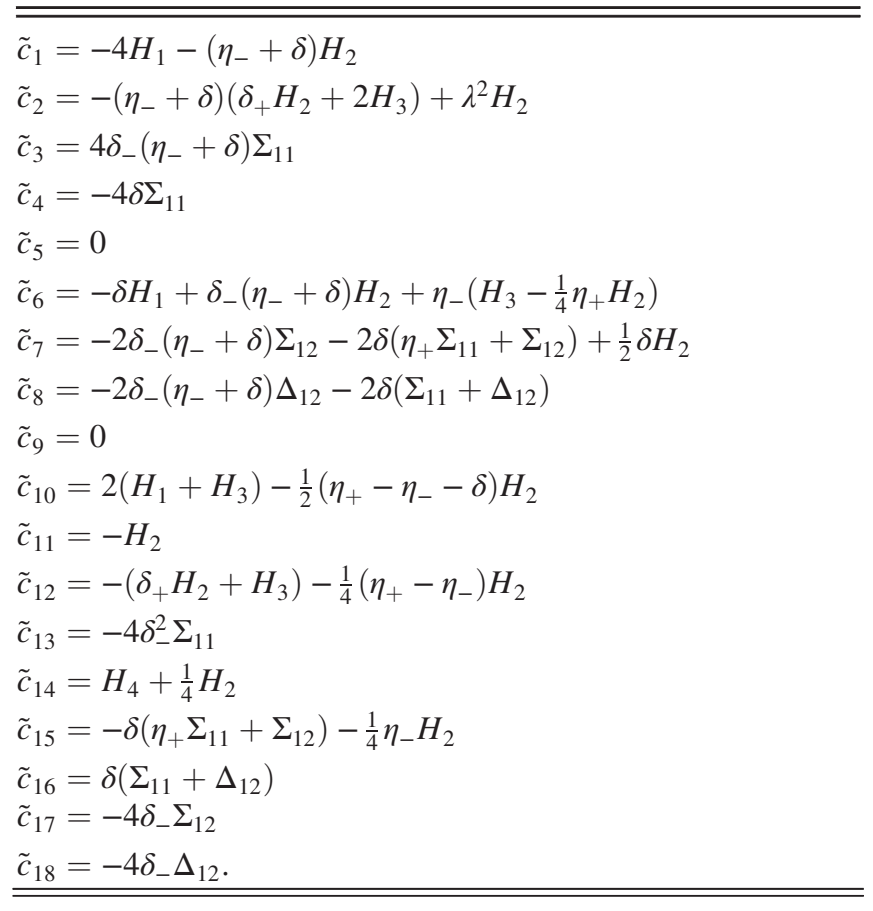

$$
D=\frac{\left(s-m_{R}^{2}\right)\left(u-m_{R}^{2}\right)}{m^{4}}=\left(\eta_{-}+\delta\right)^{2}-4 \lambda^{2}
$$

The CFF residues $\tilde{c}_{i}$ for $J^{P}=1 / 2^{+}$are given in Table VI. For $m_{R}=m$ and with the replacement $\left(Q^{2} / m^{2}\right) F_{1} \rightarrow F_{1}$ they coincide with Table IV as they should.

The case of negative-parity resonances requires no separate discussion: the vertices only differ by $\gamma_{5}$ factors, so that in the Born term (5.1) we must replace

$$
S(p \pm \Sigma) \rightarrow \gamma_{5} S(p \pm \Sigma) \gamma_{5}=S(-(p \pm \Sigma)) .
$$

With Eq. (5.10) this only amounts to a global sign switch together with an exchange $m_{R} \rightarrow-m_{R}$, because we defined the transition currents so that no $m_{R}$ factors explicitly appear therein. The CFFs for negative-parity resonances are then obtained from Table VI simply by switching all signs $\tilde{c}_{i} \rightarrow-\tilde{c}_{i}$ and exchanging $\delta_{+} \leftrightarrow-\delta_{-}$.

It is easy to work out the various kinematic limits discussed in Sec. II C:

(i) (i) In RCS $\left(\eta_{+}=\omega=0\right) H_{1}=H_{3}=0$ and

$$
H_{2}=F_{2}(0)^{2}, \quad H_{4}=-F_{1}(0) F_{2}(0)
$$

Only the CFFs $c_{1}, c_{2}, c_{6}, c_{10}, c_{11}$ and $c_{12}$ survive because the remaining tensors drop out; in the static limit where $\eta_{-}=\lambda=0$ they are related to the polarizabilities through Eqs. (2.31)-(2.33). 
(ii) In $\operatorname{VCS}\left(\eta_{+}=\omega\right)$ one has

$$
\begin{aligned}
& H_{1}=0, \quad H_{2}=F_{2}\left(Q^{2}\right) F_{2}(0), \\
& H_{3}=-\eta_{+} H_{4}=\eta_{+} F_{1}\left(Q^{2}\right) F_{2}(0) .
\end{aligned}
$$

The twelve CFFs in Eq. (2.37) survive and contribute to the generalized polarizabilities.

(iii) In the doubly-virtual forward limit $\left(\eta_{+}=\eta_{-}\right.$and $\omega=0$ ) the CFFs collapse into the four amplitudes $\bar{c}_{i}$ from Eq. (2.41). Splitting them into nonresonant and resonant terms as in (4.21), where only the latter contribute to the imaginary part, one obtains the resonance contributions to the nucleon's structure functions.

\section{SPIN-3/2 RESONANCES}

We now turn to $J^{P}=3 / 2^{ \pm}$resonances. Although the generalization to this case seems straightforward, one encounters several pathologies related to off-shell ambiguities coming from the unphysical lower-spin components in the Lorentz representations of the fields; see [47,65-69] and references therein. Such problems can be resolved by imposing spin-3/2 gauge symmetry on the effective Lagrangian [47], which leads to additional constraints for the offshell transition vertex. Here we will derive the most general off-shell spin- $1 / 2$ to spin-3/2 transition vertex that is compatible with these constraints.

The tree-level Compton amplitude with intermediate spin-3/2 resonances has the form

$$
\begin{aligned}
\Gamma_{3 / 2}^{\mu \nu}= & \Lambda_{+}\left(p_{f}\right)\left[\bar{\Gamma}_{\mathrm{R}}^{\mu \alpha}\left(p_{+}, Q^{\prime}\right) S_{\mathrm{R}}^{\alpha \beta}\left(p_{+}\right) \Gamma_{\mathrm{R}}^{\beta \nu}\left(p_{+}, Q\right)\right. \\
& \left.+\bar{\Gamma}_{\mathrm{R}}^{\nu \alpha}\left(p_{-},-Q\right) S_{\mathrm{R}}^{\alpha \beta}\left(p_{-}\right) \Gamma_{\mathrm{R}}^{\beta \mu}\left(p_{-},-Q^{\prime}\right)\right] \Lambda_{+}\left(p_{i}\right),
\end{aligned}
$$

where photon indices are denoted by $\mu, \nu$ and vector-spinor indices by $\alpha, \beta . S_{\mathrm{R}}^{\alpha \beta}(k)$ is the tree-level propagator for a spin-3/2 particle and $\Gamma_{\mathrm{R}}^{\alpha \mu}(k, Q)$ the offshell nucleon-toresonance transition vertex. From now on the argument $k$ in $\Gamma_{\mathrm{R}}^{\alpha \mu}(k, Q)$ denotes the momentum of the spin-3/2 particle and not the relative momentum, cf. Fig. 6 . We abbreviated the resonance momenta by $p_{ \pm}=p \pm \Sigma$. The chargeconjugated quantities are given by

$$
\begin{aligned}
\bar{\Gamma}_{\mathrm{R}}^{\mu \alpha}(k, Q) & =C \Gamma_{\mathrm{R}}^{\alpha \mu}(-k,-Q)^{T} C^{T}, \\
\bar{S}_{\mathrm{R}}^{\alpha \beta}(k) & =C S_{\mathrm{R}}^{\beta \alpha}(-k)^{T} C^{T}
\end{aligned}
$$

and it is straightforward to verify the Bose- and chargeconjugation invariance (2.19) of the resonance Born terms above.

The tree-level propagator for a spin-3/2 particle is the Rarita-Schwinger propagator

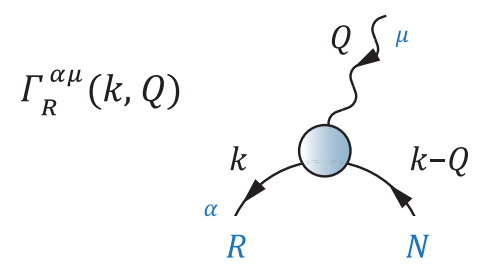

FIG. 6. Kinematics in the $N \rightarrow \frac{3}{2}^{ \pm}$transition vertex.

$$
S_{\mathrm{R}}^{\alpha \beta}(k)=\frac{-i \not k+m_{R}}{k^{2}+m_{R}^{2}} \Delta^{\alpha \beta},
$$

where $m_{R}$ is the mass of the spin-3/2 particle and the Rarita-Schwinger tensor is defined as

$$
\Delta^{\alpha \beta}=\delta^{\alpha \beta}-\frac{\gamma^{\alpha} \gamma^{\beta}}{3}+\frac{2 k^{\alpha} k^{\beta}}{3 m_{R}^{2}}+\frac{k^{\alpha} \gamma^{\beta}-\gamma^{\alpha} k^{\beta}}{3 i m_{R}} .
$$

It is well known that in the construction of vector-spinors from the Lorentz-group representations

$$
\left(\frac{1}{2}, \frac{1}{2}\right) \otimes\left[\left(\frac{1}{2}, 0\right) \oplus(\ldots)\right]=\left(\frac{1}{2}, 1\right) \oplus\left(\frac{1}{2}, 0\right) \oplus(\ldots)
$$

the spin- $3 / 2$ part is contaminated by spin- $1 / 2$ contributions from the $\left(\frac{1}{2}, 1\right)$ and $\left(\frac{1}{2}, 0\right)$ subspaces. The standard way to isolate them is to define the projectors [70]

$$
\begin{aligned}
& -\operatorname{spin}-\frac{3}{2} \operatorname{in}\left(\frac{1}{2}, 1\right): \mathbb{P}_{3 / 2}^{\alpha \beta}=P_{k}^{\alpha \beta}-\frac{1}{3} \gamma_{\perp}^{\alpha} \gamma_{\perp}^{\beta}, \\
& \text { - } \operatorname{spin}-\frac{1}{2} \operatorname{in}\left(\frac{1}{2}, 1\right): \mathbb{P}_{11}^{\alpha \beta}=\frac{1}{3} \gamma_{\perp}^{\alpha} \gamma_{\perp}^{\beta}, \\
& \text { - } \operatorname{spin}-\frac{1}{2} \operatorname{in}\left(\frac{1}{2}, 0\right): \mathbb{P}_{22}^{\alpha \beta}=\frac{k^{\alpha} k^{\beta}}{k^{2}},
\end{aligned}
$$

where $P_{k}^{\alpha \beta}=\delta^{\alpha \beta}-k^{\alpha} k^{\beta} / k^{2}$ and $\gamma_{\perp}^{\alpha}=P_{k}^{\alpha \beta} \gamma^{\beta}$ denote the transverse projector with respect to the momentum $k$ and the transverse projection of the $\gamma$-matrix (with $\gamma_{\perp}^{\alpha} \gamma_{\perp}^{\alpha}=3$ ), respectively. The spin-3/2 projector satisfies

$$
\mathbb{P}_{3 / 2}^{\alpha \beta} k^{\beta}=0, \quad \mathbb{P}_{3 / 2}^{\alpha \beta} \gamma^{\beta}=0 .
$$

The Rarita-Schwinger field can then be decomposed into

$$
\psi^{\alpha}=\left(\mathbb{P}_{3 / 2}^{\alpha \beta}+\mathbb{P}_{11}^{\alpha \beta}+\mathbb{P}_{22}^{\alpha \beta}\right) \psi^{\beta} .
$$

If we further define (note that $\gamma_{\perp}^{\alpha}$ anticommutes with $\not h$ )

$$
\mathbb{P}_{12}^{\alpha \beta}=-\frac{\gamma_{\perp}^{\alpha} k^{\beta} \not k}{\sqrt{3} k^{2}}, \quad \mathbb{P}_{21}^{\alpha \beta}=\frac{k^{\alpha} \gamma_{\perp}^{\beta} \not k}{\sqrt{3} k^{2}},
$$

the Rarita-Schwinger propagator (6.3) takes the equivalent form 


$$
\begin{aligned}
S_{\mathrm{R}}^{\alpha \beta}(k)= & \frac{-i \not k+m_{R}}{k^{2}+m_{R}^{2}} \mathbb{P}_{3 / 2}^{\alpha \beta}+\frac{2\left(-i \not k+m_{R}\right)}{3 m_{R}^{2}} \mathbb{P}_{22}^{\alpha \beta} \\
& +\frac{1}{\sqrt{3} m_{R}}\left(\mathbb{P}_{12}+\mathbb{P}_{21}\right)^{\alpha \beta} .
\end{aligned}
$$

The pole part of the propagator is proportional to $\mathbb{P}_{3 / 2}$ and corresponds to the spin- $3 / 2$ subspace. The regular terms provide the off-shell spin- $1 / 2$ background which should not contribute to matrix elements such as the Compton scattering amplitude. In addition, $\mathbb{P}_{3 / 2}$ has a kinematic singularity at $k^{2}=0$ which cannot survive in observables either.

Both problems can be resolved at the level of the offshell vertices that connect the Rarita-Schwinger propagators in matrix elements. In the case of Compton scattering this is the transition vertex $\Gamma_{\mathrm{R}}^{\alpha \mu}(k, Q)$. Demanding spin-3/2 gauge symmetry for effective Lagrangians is equivalent to imposing the transversality condition $k^{\alpha} \Gamma_{\mathrm{R}}^{\alpha \mu}(k, Q)=0$ : if both ends of the propagator (6.11) are contracted with a vertex that is transverse in $k^{\alpha}$, only the pole term $\sim \mathbb{P}_{3 / 2}$ survives because the projectors $\mathbb{P}_{22}, \mathbb{P}_{12}$ and $\mathbb{P}_{21}$ all contain instances of $k^{\alpha}$ or $k^{\beta}$. Hence, a vertex that satisfies $k^{\alpha} \Gamma_{\mathrm{R}}^{\alpha \mu}(k, Q)=0$ automatically ensures the absence of the spin-1/2 background in observables.

On the other hand, the expressions (6.3) and (6.11) do not yet represent the most general form of a spin-3/2 propagator. They follow from the kinetic term of the free Rarita-Schwinger Lagrangian $\mathcal{L}=\bar{\psi}^{\alpha} \Lambda^{\alpha \beta} \psi^{\beta}$, where $\psi^{\alpha}$ is the spin-3/2 field and $\Lambda^{\alpha \beta}$ the inverse tree-level propagator. In momentum space it takes the form

$$
\Lambda^{\alpha \beta}=-\frac{i}{2}\left\{\sigma^{\alpha \beta}, i \not k+m_{R}\right\}, \quad \sigma^{\alpha \beta}=-\frac{i}{2}\left[\gamma^{\alpha}, \gamma^{\beta}\right] .
$$

This is a special case of a family of Lagrangians which are related to each other by point transformations $[47,65,68]$; see Appendix $\mathrm{C}$ for details. The Rarita-Schwinger form corresponds to $\xi=1$, where $\xi$ is the respective gauge parameter. For $\xi \neq 1$, the general propagator is given in Eqs. (C11)-(C12): the pole part remains unchanged, but the spin- $1 / 2$ contributions depend on $\xi$ and also on the remaining projectors $\mathbb{P}_{11}$ and $\left(\mathbb{P}_{12}-\mathbb{P}_{21}\right)$. The latter still vanishes in matrix elements if the transition vertex is transverse in $k^{\alpha}$, but in order to eliminate $\mathbb{P}_{11}$ one must additionally impose $\gamma^{\alpha} \Gamma_{\mathrm{R}}^{\alpha \mu}(k, Q)=0$, which at the same time ensures the invariance of the Lagrangian under point transformations. The transversality in both $k^{\alpha}$ and $\gamma^{\alpha}$ is therefore necessary to decouple the spin- $1 / 2$ background for $\xi \neq 1$.

In summary, the resulting three constraints on the offshell vertex $\Gamma_{\mathrm{R}}^{\alpha \mu}(k, Q)$ are given by

$$
Q^{\mu} \Gamma_{\mathrm{R}}^{\alpha \mu}=0, \quad k^{\alpha} \Gamma_{\mathrm{R}}^{\alpha \mu}=0, \quad \gamma^{\alpha} \Gamma_{\mathrm{R}}^{\alpha \mu}=0 .
$$

The first incorporates electromagnetic gauge invariance; it ensures transversality with respect to $Q^{\mu}$ and therefore also on-shell current conservation. The second and third relations are automatically satisfied for the on-shell transition current due to the properties (6.8) of the projector $\mathbb{P}_{3 / 2}$ (or the Rarita-Schwinger spinors); however, for offshell generalizations of the vertex they yield additional constraints that must be worked out separately. In the 'RaritaSchwinger gauge' $\xi=1$ the first two conditions are sufficient whereas the third is only relevant for $\xi \neq 1$.

Finally, these conditions should be solved so that no kinematic singularities at $k^{\alpha}=0$ or $Q^{\mu}=0$ are introduced, which entails that $\Gamma_{\mathrm{R}}^{\alpha \mu}(k, Q)$ must be at least linear in $k^{\alpha}$ and $Q^{\mu}$. The combination of two vertices and a propagator then also cancels the kinematic $1 / k^{2}$ singularity in $\mathbb{P}_{3 / 2}$ stemming from the transverse projectors, so that all matrix elements are free of kinematic singularities. Given such a vertex, it is sufficient to employ either

$$
S_{\mathrm{R}}^{\alpha \beta}(k) \simeq \frac{-i \not k+m_{R}}{k^{2}+m_{R}^{2}} \mathbb{P}_{3 / 2}^{\alpha \beta}
$$

or the Rarita-Schwinger propagator (6.3) because both of them produce identical matrix elements.

\section{A. Off-shell $N \rightarrow \frac{3}{2}^{ \pm}$transition vertex}

To construct the general off-shell form of $\Gamma_{\mathrm{R}}^{\alpha \mu}(k, Q)$, we write down the analogue of Eq. (4.8) and collect all possible 40 tensor structures that it can contain according to Lorentz covariance and parity invariance:

$$
\left\{\begin{array}{ccc} 
& \gamma^{\alpha} k^{\mu} & k^{\alpha} k^{\mu} \\
\delta^{\alpha \mu} & k^{\alpha} \gamma^{\mu} & Q^{\alpha} Q^{\mu} \\
\gamma^{\alpha} \gamma^{\mu} & \gamma^{\alpha} Q^{\mu} & k^{\alpha} Q^{\mu} \\
& Q^{\alpha} \gamma^{\mu} & Q^{\alpha} k^{\mu}
\end{array}\right\} \times\{\mathbb{1}, \not k, Q, \not k Q\}
$$

with an extra factor $\gamma_{5}$ attached for positive-parity resonances. In analogy to the spin- $1 / 2$ case we take commutators whenever more than one $\gamma$-matrix appears in a tensor element. For example, with the definition (2.25) and the three- and four-commutators defined in Eqs. (A15)-(A16) we have

$$
\gamma^{\alpha} \gamma^{\mu} \not K Q \rightarrow\left[\gamma^{\alpha}, \gamma^{\mu}, \not K, Q\right]=-24 \varepsilon_{k Q}^{\alpha \mu}
$$

which already satisfies the first two transversality constraints in Eq. (6.13).

In analogy to the derivation of Table II, the solution of $Q^{\mu} \Gamma_{\mathrm{R}}^{\alpha \mu}=0$ and $k^{\alpha} \Gamma_{\mathrm{R}}^{\alpha \mu}=0$, where no kinematic singularities are introduced in the process, leads to the resulting 20 tensors in Table VII. Their transversality in $k^{\alpha}$ and $Q^{\mu}$ is manifest because they contain instances of $\varepsilon_{k Q}^{\alpha \mu}, t_{k \cdots}^{\alpha \cdots}$, or $t_{\cdots,} \cdots \mu$ defined in (2.25), or commutators with $\not k$ or $\emptyset$ that vanish upon contraction with $k^{\alpha}$ or $Q^{\mu}$. When inserted in the 
TABLE VII. 20 tensors for the $1 / 2^{+} \rightarrow 3 / 2^{ \pm}$transition vertex $\Gamma_{\mathrm{R}}^{\alpha \mu}(k, Q)$, Eq. (6.19), which implement the two constraints $Q^{\mu} \Gamma_{\mathrm{R}}^{\alpha \mu}=0$ and $k^{\alpha} \Gamma_{\mathrm{R}}^{\alpha \mu}=0$ without introducing kinematic singularities. Eight of them are redundant if $\gamma^{\alpha} \Gamma_{\mathrm{R}}^{\alpha \mu}=0$ is imposed as well, cf. Eq. (6.17).

\begin{tabular}{llll}
\hline \hline$m^{2} T_{1}^{\alpha \mu}=\varepsilon_{k Q}^{\alpha \mu}$ & $m^{3} T_{7}^{\alpha \mu}=i t_{k k}^{\alpha \beta} t_{\gamma Q}^{\beta \mu}$ & & \\
$m^{2} T_{2}^{\alpha \mu}=t_{k Q}^{\alpha \mu}$ & $m^{3} T_{8}^{\alpha \mu}=\frac{i}{6} t_{k Q}^{\alpha \beta}\left[\gamma^{\beta}, Q, \gamma^{\mu}\right]$ & $m^{2} T_{13}^{\alpha \mu}=\frac{1}{2}\left[t_{k \gamma}^{\alpha \beta}, t_{\gamma Q}^{\beta \mu}\right]$ & $m^{3} T_{17}^{\alpha \mu}=\frac{i}{6}\left[\gamma^{\alpha}, \not k, \gamma^{\beta}\right] t_{k Q}^{\beta \mu}$ \\
$m^{3} T_{3}^{\alpha \mu}=i t_{k \gamma}^{\alpha \beta} t_{Q Q}^{\beta \mu}$ & $m^{4} T_{9}^{\alpha \mu}=\frac{1}{2} t_{k Q}^{\alpha \beta}\left[\gamma^{\beta}, \gamma^{\nu}\right] t_{k Q}^{\nu \mu}$ & $m^{3} T_{14}^{\alpha \mu}=\frac{i}{6} t_{k k}^{\alpha \beta}\left[\gamma^{\beta}, Q, \gamma^{\mu}\right]$ & $m^{3} T_{18}^{\alpha \mu}=\frac{i}{6}\left[\gamma^{\alpha}, \not k, \gamma^{\beta}\right] t_{Q Q}^{\beta \mu}$ \\
$m^{4} T_{4}^{\alpha \mu}=t_{k k}^{\alpha \beta} t_{Q Q}^{\beta \mu}$ & $m^{4} T_{10}^{\alpha \mu}=\frac{1}{2} t_{k Q}^{\alpha \beta}\left[\gamma^{\beta}, \gamma^{\nu}\right] t_{Q Q}^{\nu \mu}$ & $m^{4} T_{15}^{\alpha \mu}=\frac{1}{2}\left[\gamma^{\alpha}, \not k k^{\beta} t_{Q Q}^{\beta \mu}\right.$ & $m^{4} T_{19}^{\alpha \mu}=\frac{1}{2} t_{k k}^{\alpha \beta}\left[\gamma^{\beta}, \gamma^{\nu}\right] t_{k Q}^{\nu \mu}$ \\
$m^{3} T_{5}^{\alpha \mu}=i t_{k Q}^{\alpha \mu} \not k$ & $m^{4} T_{11}^{\alpha \mu}=\frac{1}{2} t_{k k}^{\alpha \beta} Q^{\beta}\left[Q, \gamma^{\mu}\right]$ & $m^{5} T_{16}^{\alpha \mu}=i t_{k k}^{\alpha \beta} \gamma^{\beta} k^{\nu} t_{Q Q}^{\nu \mu}$ & $m^{4} T_{20}^{\alpha \mu}=\frac{1}{2} t_{k k}^{\alpha \beta}\left[\gamma^{\beta}, \gamma^{\nu}\right] t_{Q Q}^{\nu \mu}$ \\
$m^{3} T_{6}^{\alpha \mu}=i t_{k Q}^{\alpha \mu} Q$ & $m^{5} T_{12}^{\alpha \mu}=i t_{k Q}^{\alpha \beta} k^{\beta} \gamma^{\nu} t_{Q Q}^{\nu \mu}$ & & \\
\hline \hline
\end{tabular}

Compton amplitude, these tensors eliminate the projectors $\mathbb{P}_{22}$ and $\left(\mathbb{P}_{12}+\mathbb{P}_{21}\right)$ in the propagator so that only the spin$3 / 2$ pole part survives. ${ }^{3}$

In principle one should also work out the remaining condition $\gamma^{\alpha} \Gamma_{\mathrm{R}}^{\alpha \mu}=0$ in (6.13), which would leave 12 independent tensors. However, this is not necessary in the Rarita-Schwinger gauge $\xi=1$ because the projector $\mathbb{P}_{3 / 2}$ automatically annihilates the redundant tensors: the combinations

$$
\begin{array}{ll}
T_{13}+T_{2}-T_{1}, & T_{17}-T_{5}, \\
T_{14}-T_{7}, & T_{18}-T_{3}, \\
T_{15}, & T_{19}+\left(k^{2} / m^{2}\right) T_{2}, \\
T_{16}, & T_{20}+T_{4}
\end{array}
$$

vanish upon contraction with $\mathbb{P}_{3 / 2}$, e.g.,

$$
\mathbb{P}_{3 / 2}^{\alpha \beta}(k)\left(T_{13}^{\beta \mu}+T_{2}^{\beta \mu}-T_{1}^{\beta \mu}\right)=0 .
$$

Therefore, the first twelve elements in Table VII are sufficient when implemented in the Compton amplitude: $T_{13}$ is equivalent to $T_{1}-T_{2}$, etc. These relations hold for $J^{P}=$ $3 / 2^{ \pm}$alike because $\gamma_{5}$ commutes with the projector. The offshell $N \rightarrow 3 / 2^{ \pm}$transition vertex can then be written as

$\Gamma_{\mathrm{R}}^{\alpha \mu}(k, Q)=\left[\begin{array}{c}\gamma_{5} \\ \mathbb{1}\end{array}\right] \sum_{n=1}^{12} f_{n}^{\mathrm{R}}\left(k^{2}, k \cdot Q, Q^{2}\right) T_{n}^{\alpha \mu}(k, Q)$,

\footnotetext{
${ }^{3}$ We note that $T_{1}, T_{2}$ and $T_{3}$ in Table VII coincide with the electromagnetic couplings of the effective $N \rightarrow \Delta \gamma$ Lagrangian in Refs [36,47]:

$$
\begin{aligned}
g_{M}\left(\partial^{\mu} \bar{\psi}^{\alpha}\right) \tilde{F}^{\alpha \mu} \psi & \simeq g_{M} \bar{\psi}^{\alpha} \gamma_{5} \varepsilon_{k Q}^{\alpha \mu} A^{\mu} \psi, \\
g_{E}\left(\partial^{\mu} \bar{\psi}^{\alpha}\right) \gamma_{5} F^{\alpha \mu} \psi & \simeq g_{E} \bar{\psi}^{\alpha} \gamma_{5} t_{k Q}^{\alpha \mu} A^{\mu} \psi,
\end{aligned}
$$

and similarly for $g_{C}$. Here, $\psi^{\alpha}, \psi$ and $A^{\mu}$ are the $\Delta$, nucleon and photon fields and $F^{\mu \nu}$ is the electromagnetic field-strength tensor, with $\tilde{F}^{\mu \nu}$ its dual. For comparison, the couplings $g_{1}$ and $g_{2}$ employed in Ref. [71] correspond to $T_{13} \simeq T_{1}-T_{2}$ and $T_{2}$, respectively.
}

where the upper (lower) entry holds for resonances with positive (negative) parity.

\section{B. On-shell $N \rightarrow \frac{3}{2} \pm$ transition current}

The on-shell transition current follows from sandwiching the vertex $\Gamma_{\mathrm{R}}^{\alpha \mu}(k, Q)$ between the respective projectors and taking both momenta on-shell:

$J_{\mathrm{R}}^{\alpha \mu}=\left.\Lambda_{+}(k) \mathbb{P}_{3 / 2}^{\alpha \beta}(k) \Gamma_{\mathrm{R}}^{\beta \mu}(k, Q) \Lambda_{+}(k-Q)\right|_{\text {onshell }} \cdot$

Again, $k$ is here the outgoing momentum of the spin- $3 / 2$ resonance and $Q$ is the incoming photon momentum; the incoming nucleon momentum is $k-Q$. "On shell" refers to the kinematic limit $(k-Q)^{2}=-m^{2}$ and $k^{2}=-m_{R}^{2}$, which entails

$$
k \cdot Q=2 m^{2}\left(\tau-\frac{\delta}{4}\right) .
$$

The positive-energy projectors are

$\Lambda_{+}(k)=\frac{-i \not k+m_{R}}{2 m_{R}}, \quad \Lambda_{+}(k-Q)=\frac{-i(\not k-\mathscr{Q})+m}{2 m}$.

For $k^{2}=-m_{R}^{2}$ the two forms (6.3) and (6.11) of the propagator become equivalent:

$$
\Lambda_{+}(k) \mathbb{P}_{3 / 2}^{\alpha \beta}(k)=\Lambda_{+}(k) \Delta^{\alpha \beta}(k),
$$

so that on shell it does not matter whether we use the projector $\mathbb{P}_{3 / 2}^{\alpha \beta}(k)$ or the Rarita-Schwinger tensor $\Delta^{\alpha \beta}(k)$.

On the mass shell, the 12 structures in Table VII collapse into three tensors via the identities in Table VIII: for example, $T_{4}+r T_{3}$ vanishes in the contraction of Eq. (6.20). The on-shell current then takes the form

$$
\Gamma_{\mathrm{R}}^{\alpha \mu}(k, Q)=\left[\begin{array}{c}
\gamma_{5} \\
\mathbb{1}
\end{array}\right]\left(F_{1} T_{1}^{\alpha \mu}-F_{2} T_{2}^{\alpha \mu}-F_{3} T_{3}^{\alpha \mu}\right),
$$

which defines three dimensionless and constraint-free form factors $F_{i}\left(Q^{2}\right)$. The isospin factors are implicit in the form factors. 
TABLE VIII. Combinations of tensors that vanish in the onshell projection of Eq. (6.20) with $\Gamma_{\mathrm{R}}^{\beta \mu}(k, Q)=\gamma_{5} T_{i}^{\beta \mu}$, i.e., for resonances with $J^{P}=3 / 2^{+}$. For negative-parity resonances with $J^{P}=3 / 2^{-}$, replace $r \rightarrow-r$ and $\delta_{ \pm} \rightarrow-\delta_{\mp}$. The variables $r, \delta$ and $\delta_{ \pm}$are defined in (5.2). Whereas the combinations in Eq. (6.17) automatically also vanish on the mass shell, the expressions above do not vanish off-shell when contracted with $\mathbb{P}_{3 / 2}$.

\begin{tabular}{ll}
\hline \hline$T_{4}+r T_{3}$ & $T_{9}-2\left(\tau-\frac{1}{4} \delta\right) T_{1}-2 r \delta_{+} T_{2}$ \\
$T_{5}-r T_{2}$ & $T_{10}-4 \tau T_{1}-2 \delta_{+} T_{3}$ \\
$T_{6}-2 \delta_{+} T_{2}$ & $T_{11}-r\left(2 \delta_{-} T_{1}-2 \delta_{+} T_{2}+T_{3}\right)$ \\
$T_{7}-r\left(T_{1}+T_{2}\right)$ & $T_{12}-2 r\left(2 \tau\left(T_{1}+T_{2}\right)+\delta_{+} T_{3}\right)$ \\
$T_{8}-2 \delta_{-} T_{1}-T_{3}$ & \\
\hline \hline
\end{tabular}

With the help of Eq. (6.17) and Table VIII one can construct equivalent forms: for example, since either $T_{1}$ or $T_{2}$ can be traded for $T_{13}$ one could replace

$$
F_{1} T_{1}-F_{2} T_{2} \rightarrow F_{1} T_{13}+\left(F_{1}-F_{2}\right) T_{2}
$$

which is the combination used in Ref. [71]. The $F_{i}$ have simple relations with the form factors $g_{E}, g_{M}$ and $g_{C}$ of Ref. [36]:

$$
\left[\begin{array}{c}
g_{M} \\
g_{E} \\
g_{C}
\end{array}\right]=\sqrt{\frac{2}{3}} \frac{2 \lambda_{+}}{\delta_{+}}\left[\begin{array}{c}
F_{1} \\
F_{2} \\
r F_{3}
\end{array}\right],
$$

but due to the factor $\lambda_{+}$[defined in (5.2)] $g_{E}, g_{M}$ and $g_{C}$ have a slower falloff with $Q^{2}$ and kinematic zeros at $\lambda_{+}=$ $0 \Leftrightarrow Q^{2}=-\left(m_{R}+m\right)^{2}$.

Moreover, several equivalent forms for the on-shell current exist in the literature which are constructed from tensors different from those in Table VII. While they respect current conservation, they do not satisfy the second and third constraints in Eq. (6.13); in the diction of Ref. [47] they correspond to "inconsistent couplings" in the effective Lagrangian. An example is the $J^{P}=3 / 2^{+}$ current defined by the Jones-Scadron form factors $G_{E}^{*}, G_{M}^{*}$ and $G_{C}^{*}[48]$ :

$$
\begin{aligned}
\Gamma_{\mathrm{R}}^{\alpha \mu}= & \sqrt{\frac{3}{2}} \frac{\delta_{+}}{2 m^{4} \lambda_{+} \lambda_{-}} \gamma_{5}\left[m^{2} \lambda_{-}\left(G_{M}^{*}-G_{E}^{*}\right) \varepsilon_{k Q}^{\alpha \mu}\right. \\
& \left.-G_{E}^{*} \varepsilon_{k Q}^{\alpha \beta} \varepsilon_{k Q}^{\beta \mu}-\frac{G_{C}^{*}}{2} Q^{\alpha} k^{\beta} t_{Q Q}^{\beta \mu}\right] .
\end{aligned}
$$

The tensor for $G_{E}^{*}$ is related to Table VII via

$$
\frac{1}{m^{4}} \varepsilon_{k Q}^{\alpha \beta} \varepsilon_{k Q}^{\beta \mu}=\frac{k \cdot Q}{m^{2}} T_{2}^{\alpha \mu}-T_{4}^{\alpha \mu},
$$

but the one for $G_{C}^{*}$ has no counterpart because it violates the second condition in (6.13) and thus cannot be used off shell. On the mass shell the projector $\mathbb{P}_{3 / 2}$ enforces these constraints automatically; however, sensible off-shell generalizations must also satisfy $k^{\alpha} \Gamma_{\mathrm{R}}^{\alpha \mu}=0$ and therefore acceptable tensors must be of the form given in Table VII. The on-shell relations between the $F_{i}$ and the various conventions for $N \rightarrow 3 / 2^{ \pm}$transition form factors employed in the literature are collected in Appendix D 2.

\section{C. $J^{P}=\frac{3}{2}^{ \pm}$resonance Born terms}

We proceed by working out the resonance Born terms and resulting CFFs for $J=3 / 2^{ \pm}$resonances according to Eq. (6.1). For the off-shell vertex (6.19) we employ again the on-shell form (6.24), which depends on the three form factors $F_{i}\left(Q^{2}\right)$ that can be extracted from experiment. Concerning the propagator of the resonance we can employ either the Rarita-Schwinger form (6.3) or Eq. (6.14); both of them produce the same results because the tensors $T_{i}^{\alpha \mu}$ satisfy the required off-shell constraints.

The resulting contribution to the Compton amplitude has the form

$$
\Gamma_{3 / 2}^{\mu \nu}=\frac{1}{3 D} \sum_{i=1}^{18} \tilde{c}_{i}\left[\Lambda_{+}\left(p_{f}\right) X_{i}^{\mu \nu} \Lambda_{+}\left(p_{i}\right)\right],
$$

where the pole is given by

$$
D=\frac{\left(s-m_{R}^{2}\right)\left(u-m_{R}^{2}\right)}{m^{4}}=\left(\eta_{-}+\delta\right)^{2}-4 \lambda^{2} .
$$

The $\tilde{c}_{i}$ are the residues of the CFFs and collected in Table IX for the $J^{P}=3 / 2^{+}$case. Unfortunately the expressions become very lengthy so we only show the result for $F_{3}=0$. This form factor drops out in RCS and does not contribute to the static polarizabilities. In our numerical calculations we retain all three form factors.

In analogy to Eq. (4.21) one could rearrange the terms proportional to $\lambda^{2}$ such that the CFFs split into pole and non-pole pieces:

$$
c_{i}=\frac{\tilde{c}_{i}}{3 D}=c_{i}^{(0)}+\frac{\delta^{2} c_{i}^{(1)}}{\left(\eta_{-}+\delta\right)^{2}-4 \lambda^{2}} .
$$

In that way $c_{i}^{(0)}$ and $c_{i}^{(1)}$ depend on $\eta_{+}, \eta_{-}$and $\omega^{2}$ but no longer on $\lambda^{2}$. In Sec. VIII we will see that they effectively become functions of $\eta_{+}$only.

The various kinematic limits can be analyzed in the same way as for the $J=1 / 2$ case. The contribution from the $\Delta(1232)$ resonance was recently also worked out in the VCS limit [9] and the forward limit [72].

As before, the $J^{P}=3 / 2^{-}$case requires no separate discussion. Deleting the $\gamma_{5}$ factor in the off-shell vertex (6.19) only changes the sign of the argument in the 
TABLE IX. CFF residues for a $J^{P}=3 / 2^{+}$resonance. We only display the result for $F_{3}=0$. The necessary definitions and abbreviations are collected at the bottom. $\delta, \delta_{ \pm}$and $r$ are defined in Eq. (5.2).

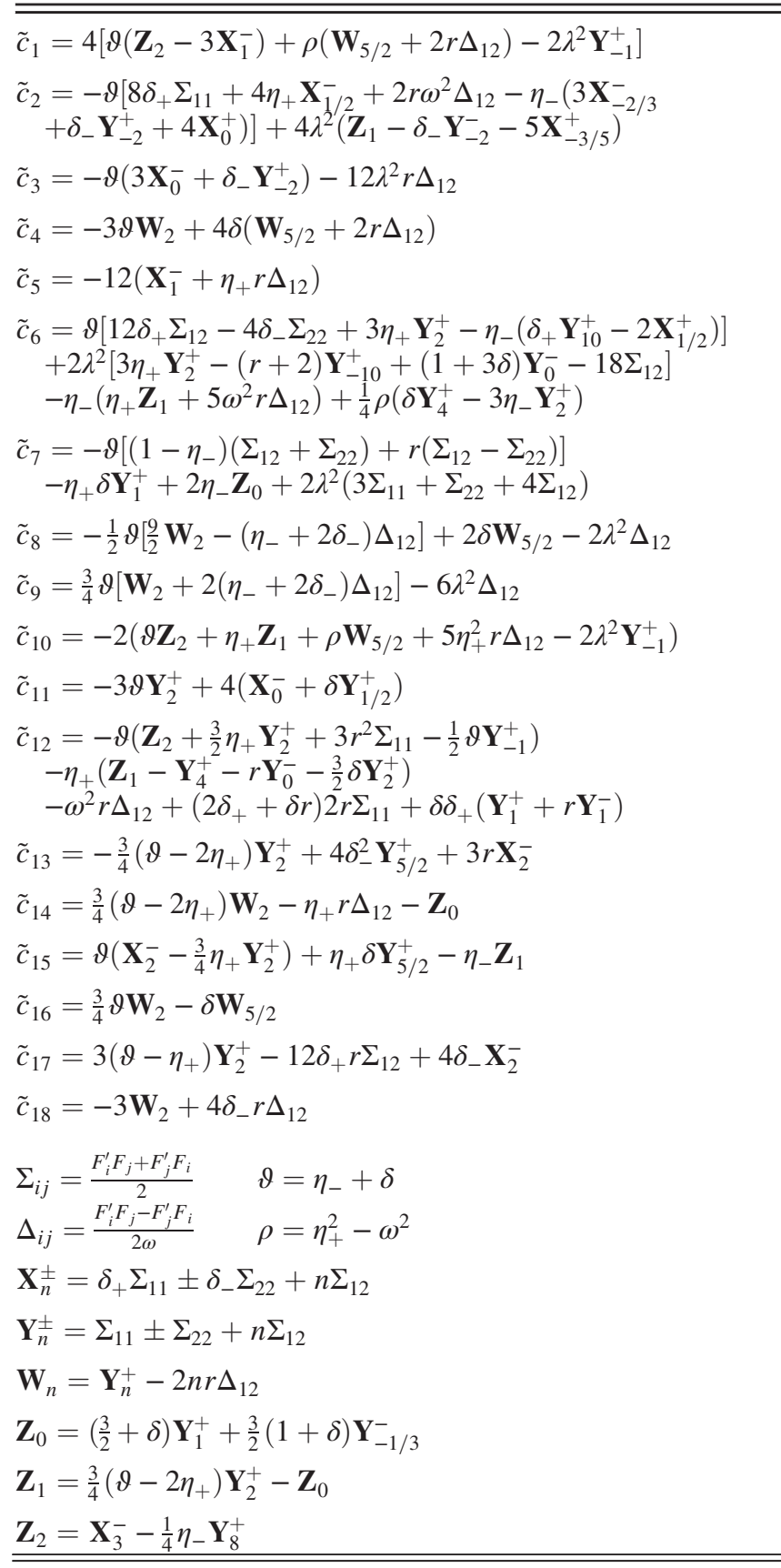

propagator as in Eq. (5.16), which amounts to replacing $m_{R} \rightarrow-m_{R}$ together with a global sign change. The CFFs for negative-parity resonances are then obtained from Table IX by replacing $r \rightarrow-r, \delta_{+} \leftrightarrow-\delta_{-}$and flipping the global sign.

The remaining task is to convert the available experimental data for the resonance electrocouplings into parametrizations for the transition form factors $F_{1,2}\left(Q^{2}\right)$ and $F_{1,2,3}\left(Q^{2}\right)$ that enter in the various transition vertices, so that they can be implemented in Compton scattering. This is what we turn to next.

\section{TRANSITION FORM FACTORS}

To work out the resonance contributions to the CFFs according to the formulas in Tables VI and IX, we need to construct parametrizations for their electromagnetic transition form factors. The currently known $J^{P}=1 / 2^{ \pm}$and $J^{P}=$ $3 / 2^{ \pm}$nucleon resonances listed in the PDG are collected in Table X. Experimental data for their $Q^{2}$-dependent electrocouplings are available for the four-star resonances [with the exception of the $\Delta(1910)]$ and the three-star resonance $N(1710)$. The data are mainly from JLab and extend up to $Q^{2}=5 \ldots 7 \mathrm{GeV}^{2}$ depending on the experiment [44,73-75]. The MAID analysis [42,76,77] also includes data from different experiments where not all multipoles are measured; the resulting parametrizations typically show some deviations from the JLab/CLAS analyses.

The experimental data are commonly presented in terms of helicity amplitudes, which are closely connected with the electroproduction amplitudes from where they are extracted [41]. To implement them in Compton scattering, however, it is mandatory to translate them into the constraint-free form factors $F_{i}\left(Q^{2}\right)$ defined by the currents (5.8) and (6.24). As explained in the previous sections, electromagnetic and spin-3/2 gauge invariance preclude using tensors others than the $T_{i}^{\mu}$ in Table II and $T_{i}^{\alpha \mu}$ in Table VII for the transition currents. For example, using the Jones-Scadron basis in Eq. (6.27) or the helicity basis in Eq. (D14) can lead to spurious singularities in the CFFs.

Furthermore, the helicity amplitudes are subject to timelike kinematic constraints, which typically also lead to complicated momentum dependencies in the spacelike region $Q^{2}>0$. By contrast, the $F_{i}\left(Q^{2}\right)$ are kinematically independent and thus simpler: without kinematic effects, their momentum dependence is governed by physical

TABLE X. Two- to four-star nucleon and $\Delta$ resonances below $2 \mathrm{GeV}$ for $J^{P}=\frac{1}{2}^{ \pm}$and $\frac{3}{2}^{ \pm}$[78]. The four-star resonances are shown in bold font. In a spectroscopic notation they are labeled by the incoming partial wave $L_{2 I, 2 J}$ in elastic $\pi N$ scattering; from left to right: $P_{11}, P_{13}, S_{11}, D_{13}$ for the nucleon resonances with $I=\frac{1}{2}$ and $P_{31}, P_{33}, S_{31}, D_{33}$ for the $\Delta$ resonances with $I=\frac{3}{2}$.

\begin{tabular}{lccc}
\hline \hline$J^{P}=\frac{1}{2}^{+}$ & $\frac{3}{2}^{+}$ & $\frac{1}{2}^{-}$ & $\frac{3}{2}{ }^{-}$ \\
$\mathbf{N}(\mathbf{9 4 0})$ & $\mathbf{N}(\mathbf{1 7 2 0})$ & $\mathbf{N}(\mathbf{1 5 3 5})$ & $\mathbf{N}(\mathbf{1 5 2 0})$ \\
$\mathbf{N}(\mathbf{1 4 4 0})$ & $N(1900)$ & $\mathbf{N}(\mathbf{1 6 5 0})$ & $N(1700)$ \\
$N(1710)$ & & $N(1895)$ & $N(1875)$ \\
$N(1880)$ & & & \\
$\boldsymbol{\Delta}(\mathbf{1 9 1 0})$ & $\boldsymbol{\Delta}(\mathbf{1 2 3 2})$ & $\mathbf{\Delta}(\mathbf{1 6 2 0})$ & $\mathbf{\Delta}(\mathbf{1 7 0 0})$ \\
& $\Delta(1600)$ & $\Delta(1900)$ & $\Delta(1940)$ \\
& $\Delta(1920)$ & & \\
\hline \hline
\end{tabular}


singularities, namely the pion production cuts at timelike values $Q^{2}<-4 m_{\pi}^{2}$ and vector-meson poles in the complex plane. Up to logarithmic corrections, the form factors follow a multipole behavior at large $Q^{2}$ [79-82]. For decreasing $Q^{2}$ it is then reasonable to expect a monotonous increase towards the nearest $\rho$-meson pole, which is the closest non-analyticity relevant for the spacelike region. In the absence of resonance dynamics, the vector-meson poles would appear on the timelike real $Q^{2}$ axis (cf. Sec. 4.2 in Ref. [28] for a discussion of the explicit mechanism). The cuts signal the onset of pioncloud effects, which push the poles onto higher Riemann sheets and induce deviations from monotonicity at low $Q^{2}>0$. This is our guiding assumption for ground states, whereas for excited states some form factors will naturally have zero crossings for $Q^{2}>0$.

A simple parametrization that is flexible enough to accommodate these features is

$$
F\left(Q^{2}\right)=\frac{1}{1+x} \frac{1}{(1+y)^{n-1}}(H(x) \pm E(x)),
$$

where $x=Q^{2} / m_{\rho}^{2}, y=Q^{2} / m_{R}^{2}$ and

$$
\begin{aligned}
& H(x)=\frac{a_{0}+a_{1} x+a_{2} b_{2} x^{2}}{1+b_{2} x^{2}}, \\
& E(x)=e_{0} \sqrt{1+e_{1} x^{2}} .
\end{aligned}
$$

$E(x)$ defines the error estimate. $F\left(Q^{2}\right)$ depends on two scales, the $\rho$-meson mass and the resonance mass $m_{R}$. While all form factors should have vector-meson poles, the additional poles in $m_{R}$ effectively implement the proper multipole falloff at large $Q^{2}$, with $n=3$ or $n=4$ depending on the form factor. For ground states the remainders $H(x)$ should then become roughly constant; they approach the constant values $a_{0}$ for $Q^{2}=0$ and $a_{2}$ for $Q^{2} \rightarrow \infty$. In most cases it is sufficient to set $a_{1}=0$. We assume that $a_{0}$ and $a_{2}$ have the same sign, except for form factors with zero crossings, and we demand $b_{2}>0$ to avoid extra singularities. Although this form has no particle production cuts and only one $\rho$ pole on the real axis (which can be easily remedied by introducing a width), it does capture the spacelike properties reasonably well, in particular in the low- and intermediate $Q^{2}$ region where experimental data exist.

In practice we convert the experimental data for the helicity amplitudes to the form factors $F_{i}\left(Q^{2}\right)$, using the relations in Appendix D, and divide out the poles in Eq. (7.1) so that the data and their error bars are given in terms of $H_{x} \pm \Delta H_{x}$. Those we subsequently fit by the function $H(x)$ given above. To arrive at the uncertainty bands shown in the plots, we fit

$$
\sqrt{\left(H_{x}-H(x)\right)^{2}+\left(\Delta H_{x}\right)^{2}}
$$

by the rather conservative ansatz $E(x)$ : in that way, the error bands grow linearly at large $Q^{2}$ (unless $e_{1}=0$ ) so that the form factors can change their multipole falloff by one power of $Q^{2}$ within the uncertainty. We prefer this form because in several cases the asymptotic powers at large $Q^{2}$ are under dispute and logarithmic corrections can modify them as well.

At the $Q^{2}=0$ point we use the PDG 2016 estimates for the helicity amplitudes from photoproduction experiments [78]. For the electroproduction data at $Q^{2}>0$ we only included data sets which measure the complete set of helicity amplitudes, because otherwise one cannot extract all form factors. Whereas for the lowest-lying resonances $-\Delta(1232)$, $N(1440), N(1520)$ and $N(1535)$ - sufficient data are available, the data sets for the higher-lying resonances are scarce so that in those cases the fits are only qualitative. In addition, with the exception of the $\Delta(1232)$ all cases suffer from the lack of data below $Q^{2} \lesssim 0.3 \mathrm{GeV}^{2}$. This is unfortunate because the most important CFF contributions come from the region at low momenta, which in some cases are difficult to parametrize. This clearly motivates the need for future measurements at low $Q^{2}$.

Our resulting fits for the form factors and helicity amplitudes are shown in Figs. 7-15, where they are represented by solid lines with bands. The dashed (blue) lines are the MAID parametrizations [42,76,77] which are included for comparison. The fit parameters are collected in Tables XI and XII. For the parameters $m_{R}$ entering in the fits we simply used the names in Table X, e.g., $m_{R}=1.535 \mathrm{GeV}$ for the $N(1535)$ resonance, and we employed $m_{\rho}=0.77 \mathrm{GeV}$. In the following we discuss the resonance transition form factors one by one.

\section{A. States with $J^{P}=1 / 2^{ \pm}$}

In these cases there are two transition form factors, the Dirac-like $F_{1}\left(Q^{2}\right)$ and Pauli-like $F_{2}\left(Q^{2}\right)$ form factor. As discussed in connection with Eq. (5.9), our $F_{1}$ differs from the standard convention $F_{1}^{*}$ by a factor $Q^{2} / \mathrm{m}^{2}$ which removes its kinematic zero at $Q^{2}=0$. From the figures one

TABLE XI. Fit parameters for the $J^{P}=1 / 2^{ \pm}$resonance transition form factors.

\begin{tabular}{lccccccccc}
\hline \hline & & $n$ & $a_{0}$ & $a_{1}$ & $a_{2}$ & $b_{2}$ & $e_{0}$ & $e_{1}$ & $\chi^{2}$ \\
\hline$N(1440)$ & $F_{1}$ & 3 & 0.28 & 0.71 & 0.25 & 0.06 & 0.14 & 0.55 \\
& $F_{2}$ & 3 & -0.45 & 1.92 & 0.22 & 0.05 & 8.36 & 1.16 \\
$N(1710)$ & $F_{1}$ & 3 & -0.04 & & & 0.06 & 0.02 & 0.80 \\
& $F_{2}$ & 3 & 0.35 & & & 0.12 & 0.12 & 1.34 \\
$N(1535)$ & $F_{1}$ & 3 & 0.56 & 0.85 & 0.46 & 0.03 & 0.42 & 0.29 \\
& $F_{2}$ & 3 & -0.69 & -0.07 & 0.47 & 0.06 & 2.18 & 1.32 \\
$N(1650)$ & $F_{1}$ & 3 & 0.33 & & & 0.09 & 0.47 & 25.7 \\
& $F_{2}$ & 3 & -0.30 & & & 0.04 & 7.86 & 5.8 \\
$\Delta(1620)$ & $F_{1}$ & 3 & 0.25 & & & 0.13 & 0.00 & 4.32 \\
& $F_{2}$ & 3 & -0.06 & & & 0.37 & 0.02 & 18.0 \\
\hline \hline
\end{tabular}


TABLE XII. Fit parameters for the $J^{P}=3 / 2^{ \pm}$resonance transition form factors.

\begin{tabular}{lccrcccccc}
\hline \hline & & $n$ & $a_{0}$ & $a_{1}$ & $a_{2}$ & $b_{2}$ & $e_{0}$ & $e_{1}$ & $\chi^{2}$ \\
\hline$\Delta(1232)$ & $F_{1}$ & 3 & 1.53 & & 0.87 & 0.04 & 0.06 & 0.02 & 1.89 \\
& $F_{2}$ & 3 & -0.59 & & -0.25 & 0.11 & 0.08 & 0.00 & 0.83 \\
& $F_{3}$ & 4 & 0.29 & 1.22 & 0.01 & 0.21 & 0.00 & 0.37 \\
$\Delta(1700)$ & $F_{1}$ & 3 & -0.31 & & & & 0.30 & 0.00 & 7.14 \\
& $F_{2}$ & 3 & -0.27 & & & & 0.21 & 0.00 & 4.55 \\
& $F_{3}$ & 4 & -0.19 & & & & 0.09 & 0.00 & 11.0 \\
$N(1520)$ & $F_{1}$ & 3 & 1.42 & & 1.09 & 0.03 & 0.16 & 0.03 & 1.43 \\
& $F_{2}$ & 3 & -0.20 & 1.03 & -0.23 & 0.94 & 0.03 & 0.80 & 0.68 \\
& $F_{3}$ & 4 & -0.35 & 0.21 & 0.61 & 0.50 & 0.08 & 0.16 & 1.11 \\
$N(1720)$ & $F_{1}$ & 3 & -0.05 & & & & 0.17 & 0.30 & 9.78 \\
& $F_{2}$ & 3 & 0.13 & & & & 0.39 & 0.00 & 1.85 \\
& $F_{3}$ & 4 & 1.14 & & & & 0.53 & 0.00 & 8.26 \\
\hline \hline
\end{tabular}

can see that in most cases $F_{1}$ is indeed compatible with a monotonous rise towards $Q^{2} \rightarrow 0$.

The relations between the form factors and helicity amplitudes $A_{1 / 2}$ and $S_{1 / 2}$ are given in Eqs. (D9)-(D10). They imply in particular that at the pseudothreshold (the Siegert limit [90]) where

$$
|\mathbf{k}|=0 \Leftrightarrow Q^{2}=-\left(m_{R}-m\right)^{2},
$$

with $|\mathbf{k}|$ denoting the virtual photon three-momentum in the resonance rest frame and defined in Eq. (D4), the helicity amplitudes behave as [91-93]

$$
\begin{aligned}
& J^{P}=1 / 2^{+}: A_{1 / 2} \propto|\mathbf{k}|, \quad S_{1 / 2} \propto|\mathbf{k}|^{2}, \\
& J^{P}=1 / 2^{-}: S_{1 / 2} \propto|\mathbf{k}| .
\end{aligned}
$$

For larger timelike momenta they become imaginary. Without knowledge of the constraint-free form factors these features would not be evident, whereas they are automatic if one starts directly from the $F_{i}$. As a consequence, even simple monotonous ansätze for the $F_{i}$ typically lead to complicated shapes for the helicity amplitudes, as can be seen in the figures below.
$N(1440)$ : The Roper resonance is the first excitation in the $(I) J^{P}=(1 / 2) 1 / 2^{+}$channel. As such, $F_{2}$ has a zero crossing at intermediate $Q^{2}$, which is visible in Fig. 7 and also found in theoretical calculations [94-100]. By contrast, the data for $F_{1}$ agree with a monotonous rise. The MAID parametrizations implement a vanishing $F_{1}^{*}(0)=0$, however with a negative derivative; this implies a small negative value for $F_{1}(0)$ which produces the turnover at low $Q^{2}$ in the leftmost panel of Fig. 7. In the helicity amplitudes the difference is visible in $S_{1 / 2}$, where MAID is compatible with the recent A1/MAMI measurement for $S_{1 / 2}$ at very low $Q^{2}$ [83] but does not reproduce the behavior (7.5) at the pseudothreshold. These relations follow automatically when we parametrize the form factors directly, as can be seen in the plots.

$N(1710)$ : Since this is the second excited state in the $(I) J^{P}=(1 / 2) 1 / 2^{+}$channel, one might expect two zero crossings in $F_{2}$. The five points in Fig. 8 are recent data from $\mathrm{JLab}$; they may indicate a slight trend in that direction but are too sparse to draw conclusions. $F_{1}$ is very small. In this case we simply fit the $H_{x}$ in Eq. (7.1) to constants by setting $a_{2}=b_{2}=0$. Also here the resulting helicity amplitudes have sharp turnovers at the respective pseudothreshold $Q^{2}=$ $-\left(m_{R}-m\right)^{2}$, which lies outside of the displayed region.

$N(1535)$ : The parity partner of the nucleon is the ground state in the $(I) J^{P}=(1 / 2) 1 / 2^{-}$channel and so we expect a monotonous behavior for both form factors, which is indeed visible in Fig. 9. As noted in Refs. [101-103], the magnitude of $F_{2}$ quickly falls off with $Q^{2}$ and is compatible with zero above $Q^{2} \approx 1.5 \mathrm{GeV}^{2}$. In Table XI this amounts to the coefficient $a_{2}$, which dominates at large $Q^{2}$, being small compared to $a_{0}$. Model calculations typically yield values of $F_{2}$ with a different sign compared to the data $[101,104]$ but they also do not include the $\rho$ pole; this may suggest cancellation effects between the vectormeson pole contributions and the remainder, or also large meson-cloud contributions at low $Q^{2}$ [103]. The oscillatory behavior of $S_{1 / 2}$ near the pseudothreshold is again a consequence of Eq. (7.5).
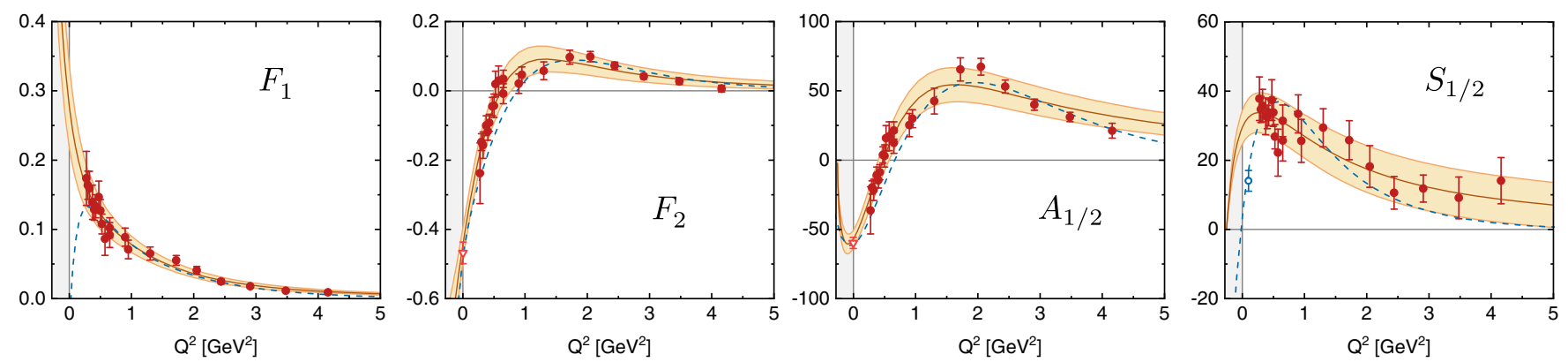

FIG. 7. Parametrization of the $\gamma^{*} N \rightarrow N(1440)$ form factors and helicity amplitudes (solid lines with bands). The data points at $Q^{2}=0$ are from the PDG [78] and those for $Q^{2}>0$ from CLAS/JLab [44,73,74]. For $S_{1 / 2}$ we also include the A1/MAMI point at $Q^{2} \simeq 0.1 \mathrm{GeV}^{2}$ [83]. The MAID parametrization (dashed, blue) is from Refs. [42,76,77]. The form factors are dimensionless and the helicity amplitudes carry units of $10^{-3} \mathrm{GeV}^{-1 / 2}$. 

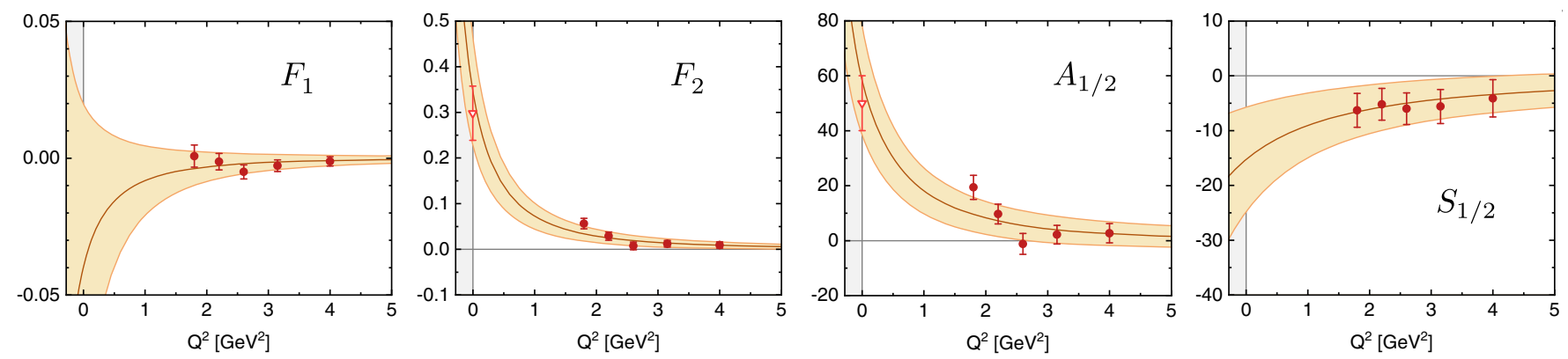

FIG. 8. Same as Fig. 7 but for $\gamma^{*} N \rightarrow N(1710)$. The data are from PDG [78] and CLAS/JLab [84].
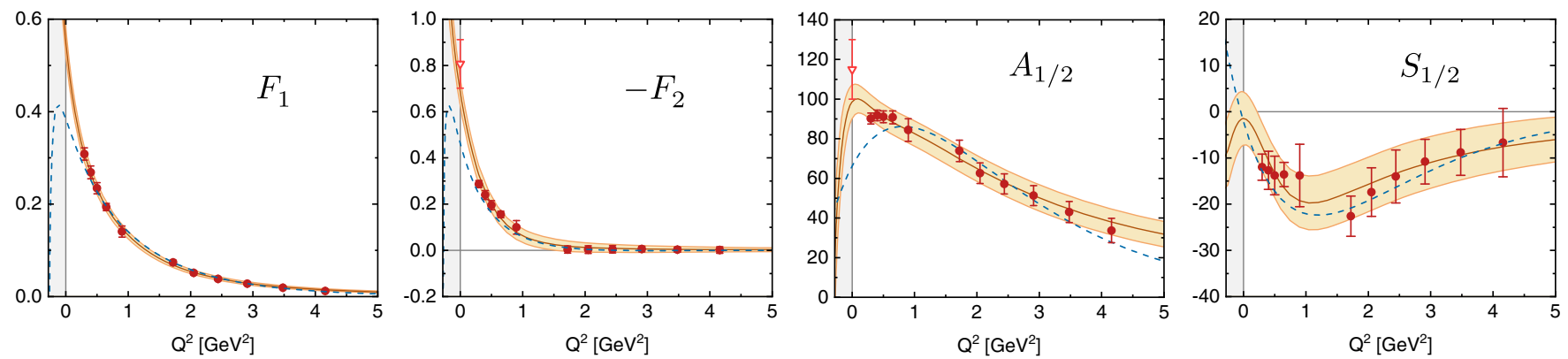

FIG. 9. Same as Fig. 7 but for $\gamma^{*} N \rightarrow N(1535)$. The data are from PDG [78] and CLAS/JLab [73].
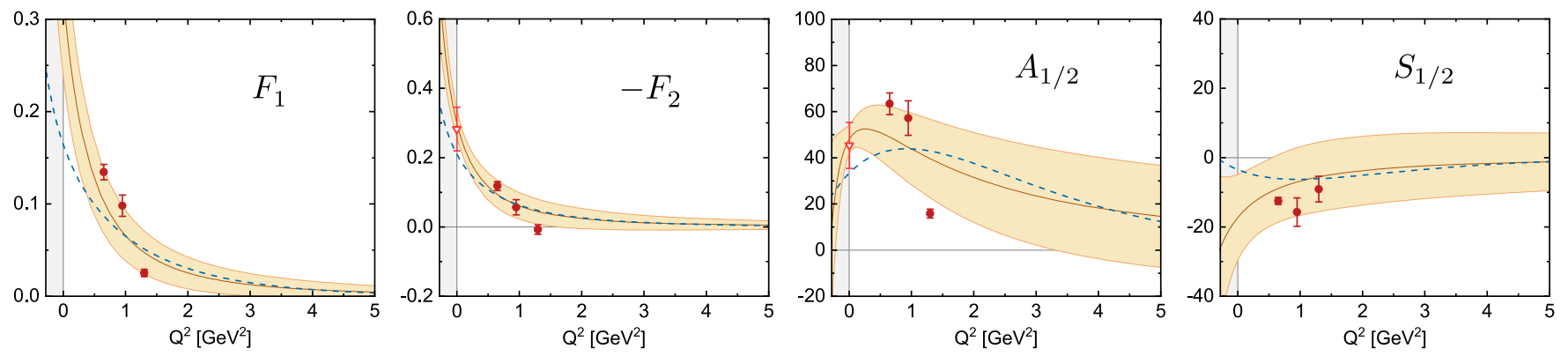

FIG. 10. Same as Fig. 7 but for $\gamma^{*} N \rightarrow N(1650)$. The data are from PDG [78] and CLAS/JLab [85].

$N(1650)$ : The first excited state in the $(I) J^{P}=$ $(1 / 2) 1 / 2^{-}$channel is shown in Fig. 10. So far there are only three data points from JLab. $F_{2}$ may be compatible with a zero crossing but in the absence of data we fit both $H_{x}$ to constants.
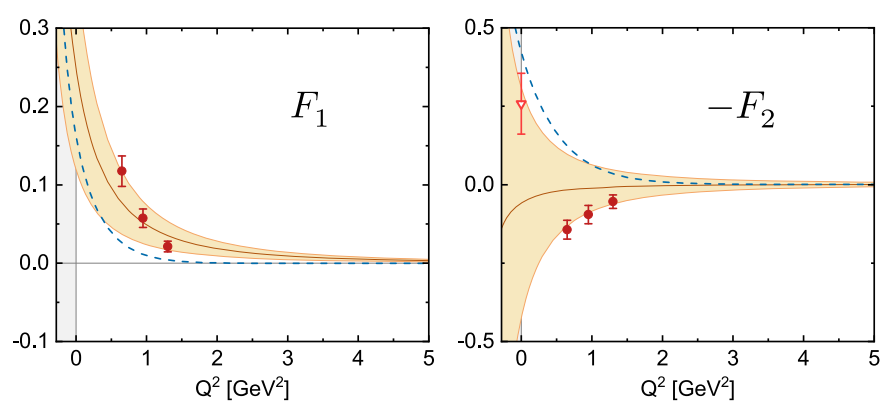

$\Delta(1620)$ : Also for the $(I) J^{P}=(3 / 2) 1 / 2^{-}$ground state the data are sparse. In addition, Fig. 11 displays some tension between the two data sets for $F_{2}\left(Q^{2}\right)$ : the three JLab points rise towards a negative value at $Q^{2}=0$ whereas the PDG estimate is positive. Studies of
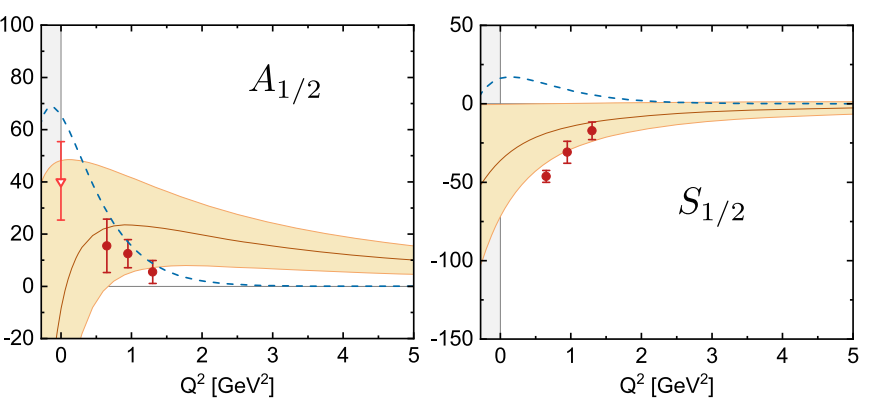

FIG. 11. Same as Fig. 7 but for $\gamma^{*} N \rightarrow \Delta(1620)$. The data are from PDG [78] and CLAS/JLab [85]. 
negative-parity states suggest a falloff $F_{1}\left(Q^{2}\right) \propto 1 / Q^{8}$ at large $Q^{2}$ due to the suppression of valence-quark contributions, which corresponds to $A_{1 / 2}\left(Q^{2}\right) \propto 1 / Q^{5}$ [105]. Due to the lack of data we take a neutral point of view and fit the $H_{x}$ again by constants, so that the resulting parametrizations implement the usual $\propto 1 / Q^{6}$ falloff.

\section{B. States with $J^{P}=3 / 2^{ \pm}$}

The $J^{P}=3 / 2^{ \pm}$resonances are determined by three transition form factors $F_{i}\left(Q^{2}\right)$ or, equivalently, the helicity amplitudes $A_{3 / 2}\left(Q^{2}\right), A_{1 / 2}\left(Q^{2}\right)$ and $S_{1 / 2}\left(Q^{2}\right)$. Their relations with the form factors are given in Eqs. (D15)-(D16). It is also common to express them in terms of the Jones-Scadron form factors [48,49]: magnetic dipole $G_{M}^{*}$, electric quadrupole $G_{E}^{*}$, and Coulomb quadrupole $G_{C}^{*}$; see Eq. (D20) for their relations with the $F_{i}$. Their electromagnetic ratios are defined as

$$
R_{\mathrm{EM}}=-\frac{G_{E}^{*}}{G_{M}^{*}}, \quad R_{\mathrm{SM}}=-\frac{|\mathbf{k}|}{2 m_{R}} \frac{G_{C}^{*}}{G_{M}^{*}}
$$

At the pseudothreshold $|\mathbf{k}|=0$, the helicity amplitudes satisfy the constraints $[91,92,106]$

$$
\begin{aligned}
& J^{P}=3 / 2^{+}: A_{3 / 2}, A_{1 / 2} \propto|\mathbf{k}|, \quad S_{1 / 2} \propto|\mathbf{k}|^{2}, \\
& J^{P}=3 / 2^{-}: S_{1 / 2} \propto|\mathbf{k}|
\end{aligned}
$$

which are a direct consequence of Eqs. (D13) and (D16). Likewise, the kinematic relations between the JonesScadron form factors at the pseudothreshold follow from the definition (7.6) and Eq. (D20):

$$
\begin{aligned}
J^{P} & =3 / 2^{+}: 2 m G_{E}^{*}-\left(m_{R}-m\right) G_{C}^{*} \propto|\mathbf{k}|^{2}, \quad R_{\mathrm{SM}} \propto|\mathbf{k}|, \\
J^{P} & =3 / 2^{-}: G_{M}^{*} \propto|\mathbf{k}|^{2}, \quad m G_{E}^{*}+\left(m_{R}-m\right) G_{C}^{*} \propto|\mathbf{k}|^{2}, \\
R_{\mathrm{EM}} & \propto 1 /|\mathbf{k}|^{2}, \quad R_{\mathrm{SM}} \propto|\mathbf{k}| .
\end{aligned}
$$

We emphasize again that the $F_{i}\left(Q^{2}\right)$ are free of kinematic constraints.

At asymptotically large $Q^{2}$, the structure of the transition currents implies the relation $G_{M}^{*} \simeq-G_{E}^{*}$, which amounts to $R_{\mathrm{EM}} \rightarrow 1$ [82]. From Eq. (D20) one obtains

$$
R_{\mathrm{EM}} \stackrel{Q^{2} \rightarrow \infty}{\rightarrow} \begin{cases}-F_{2} /\left(2 F_{1}+F_{2}\right) & \ldots J^{P}=3 / 2^{+} \\ -\left(F_{1}+2 F_{2}\right) / F_{1} & \ldots J^{P}=3 / 2^{-}\end{cases}
$$

and thus $F_{1} \simeq-F_{2}$ in both cases. In terms of the helicity amplitudes this entails a dominance of $A_{1 / 2}$ over $A_{3 / 2}$ $[48,49,74,81]$. We chose not to enforce this property in our fits (i.e., by constraining the respective coefficients $a_{2}$ ) because it is effectively absorbed in our error bands which grow with $Q^{2}$. The onset of such behavior may very well happen only at very large $Q^{2}$ and logarithmic corrections may spoil it; and with the exception of the $\Delta(1232)$ the available data are in certain conflict with the constraint.

$\Delta(1232)$ : The $\Delta$ resonance with $(I) J^{P}=(3 / 2) 3 / 2^{+}$is the lowest-lying and best known nucleon resonance, both in terms of precision and $Q^{2}$ range. A significant amount of data for its helicity amplitudes have been collected in several experiments [73,86-89,107]. For our fits we used the comprehensive database of Ref. [75] but replaced the older data for $Q^{2}<0.2 \mathrm{GeV}^{2}$ by the most recent analysis from Ref. [89]. At $Q^{2}=0$ we use the PDG estimate [78].

Figure 12 shows that $F_{1}$ and $F_{2}$ are well described by simple monotonous multipole functions. For $F_{3}$ the situation is less clear due to the low- $Q^{2}$ data, but since they come with large error bars our fit still returns a positive value for $a_{0}$ and thus a monotonous function. The resulting helicity amplitudes are plotted in the second row and they all vanish at the pseudothreshold.

The $\gamma^{*} N \rightarrow \Delta(1232)$ transition is usually discussed in terms of the Jones-Scadron form factors displayed in the third row of Fig. 12. At $Q^{2}=0$, Eq. (D20) entails that

$$
\begin{aligned}
G_{M}^{*} & =\sqrt{\frac{2}{3}\left(2 \delta_{+} F_{1}-\delta_{-} F_{2}\right),} \\
{\left[\begin{array}{c}
R_{\mathrm{EM}} \\
R_{\mathrm{SM}}
\end{array}\right] } & =\frac{\delta}{8 \delta_{+}^{2} F_{1}-\delta F_{2}}\left[\begin{array}{c}
F_{2} \\
F_{2}+\frac{\delta}{2 r} F_{3}
\end{array}\right],
\end{aligned}
$$

where $\delta, \delta_{ \pm}$and $r$ are defined in Eq. (5.2). With $\delta_{+} \gg \delta_{-}$, the dominance of the magnetic dipole form factor $G_{M}^{*}$ then translates into the dominance of $F_{1}$, whereas $F_{2}$ and $F_{3}$ enter in the small ratios. Note that $R_{\mathrm{SM}}$ must vanish at the pseudothreshold due to Eq. (7.8).

Quark models can explain the dominance of $G_{M}^{*}$ but typically underestimate its magnitude by about $30-40 \%$ at low $Q^{2}$ [108-113]. In dynamical coupled-channel models that gap is usually attributed to meson-cloud effects [110-112]. Model calculations and large- $N_{c}$ estimates also suggest a small valence-quark contribution to $R_{\mathrm{EM}}$ and $R_{\mathrm{SM}}$, indicating that these ratios may be dominated by pion-cloud effects [113-117]. By contrast, in Dyson-Schwinger calculations the valence-quark components are significant due to relativistic effects $[28,118$ 120]. Equation (7.9) shows that in the absence of $F_{2}$ and $F_{3}$ also $R_{\mathrm{EM}}(0)$ and $R_{\mathrm{SM}}(0)$ must vanish at $Q^{2}=0$, and if $R_{\mathrm{EM}}$ were mainly a pion-cloud effect the same would be true for $F_{2}$. Concerning $F_{3}$, large- $N_{c}$ estimates predict $F_{3}(0) \simeq 0$ and thus $R_{\mathrm{SM}}(0) \simeq R_{\mathrm{EM}}(0) \quad[89,115,117]$. In large- $N_{c}$ based meson cloud models $[115-117,121] F_{3}$ is small and negative below $Q^{2}=0$. 

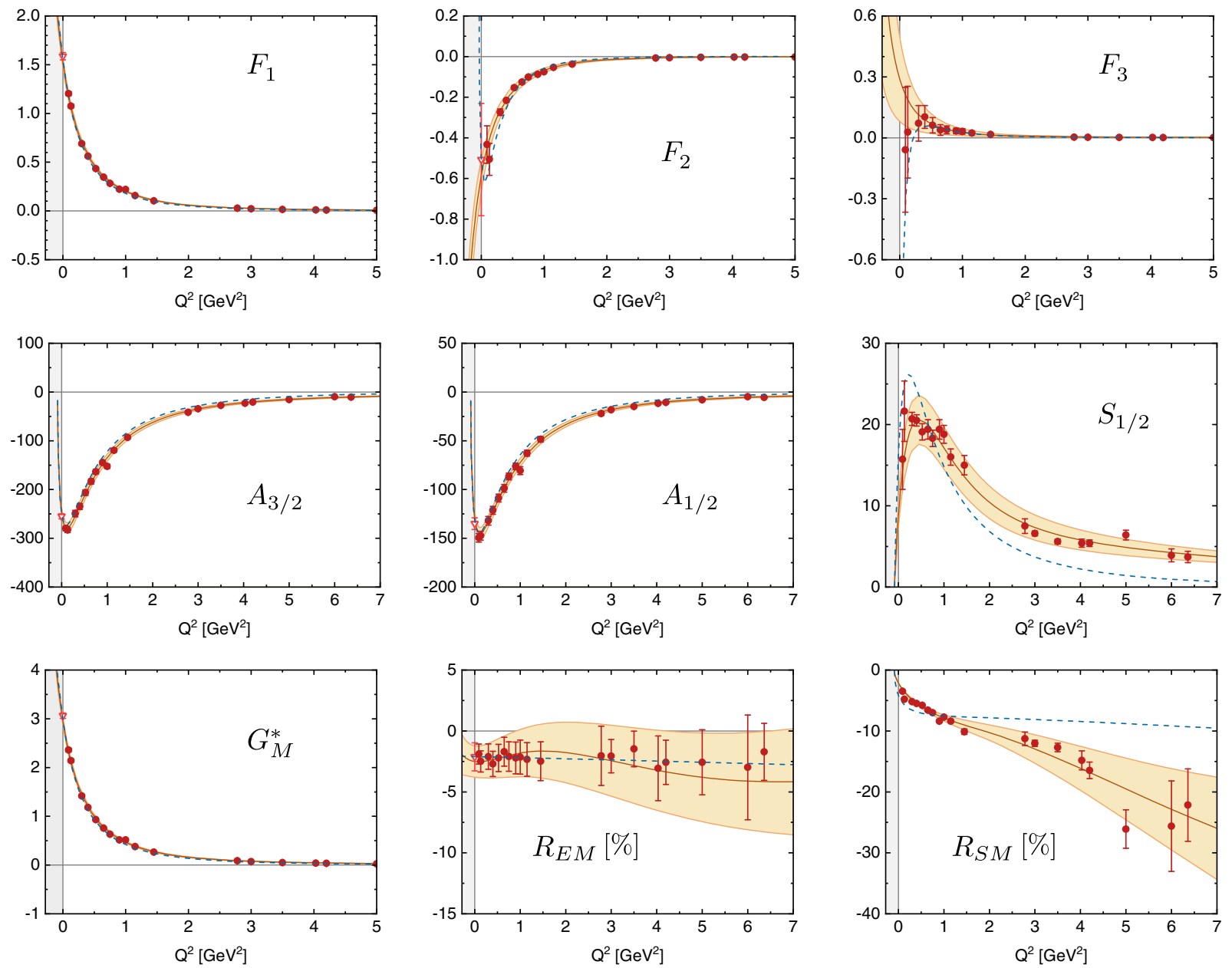

FIG. 12. $\gamma^{*} N \rightarrow \Delta(1232)$ transition form factors and helicity amplitudes. The data are from Refs. [73,78,86-89]. The form factors are dimensionless and the helicity amplitudes carry units of $10^{-3} \mathrm{GeV}^{-1 / 2}$.
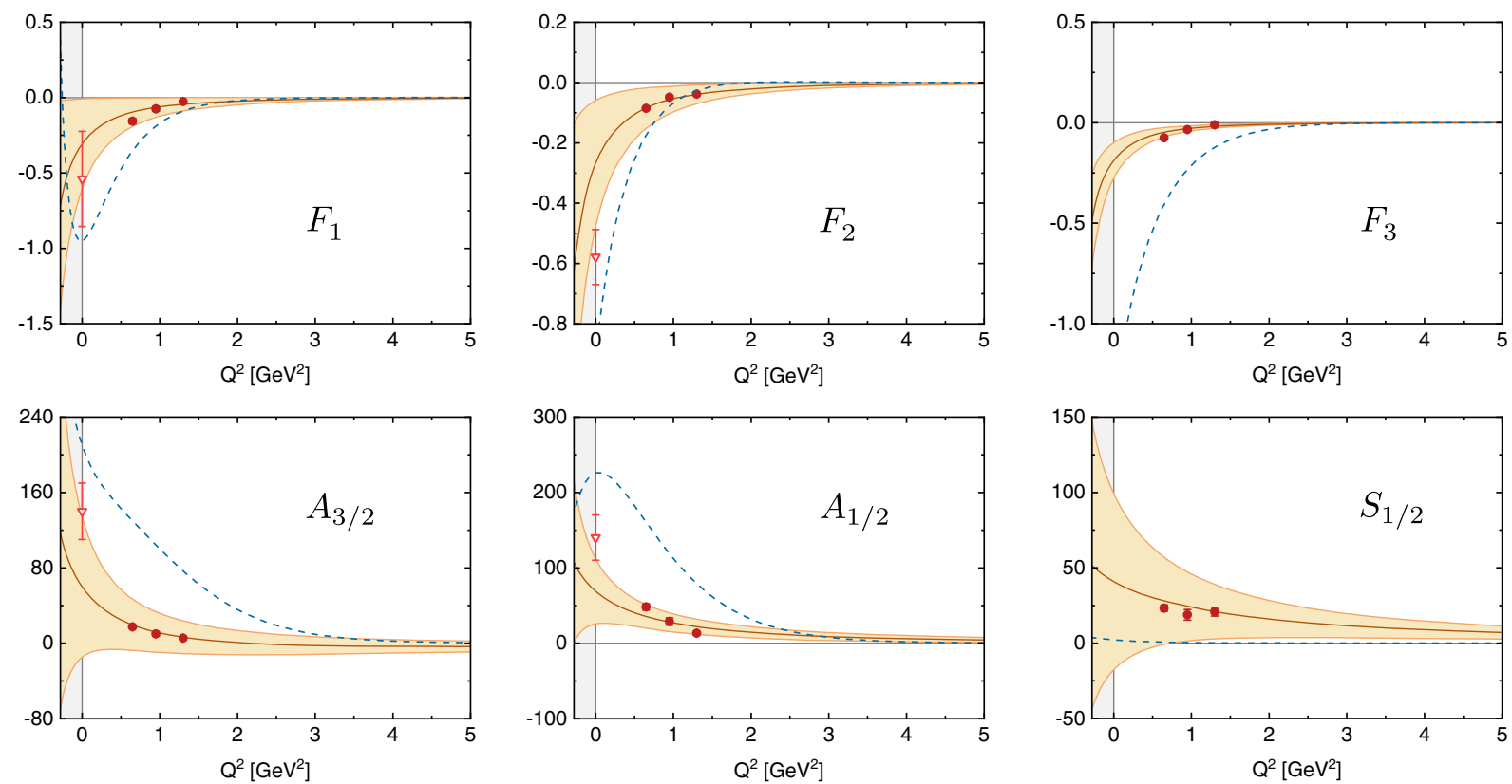

FIG. 13. Same as Fig. 12 but for $\gamma^{*} N \rightarrow \Delta(1700)$. The data are from PDG [78] and CLAS/JLab [85]. 

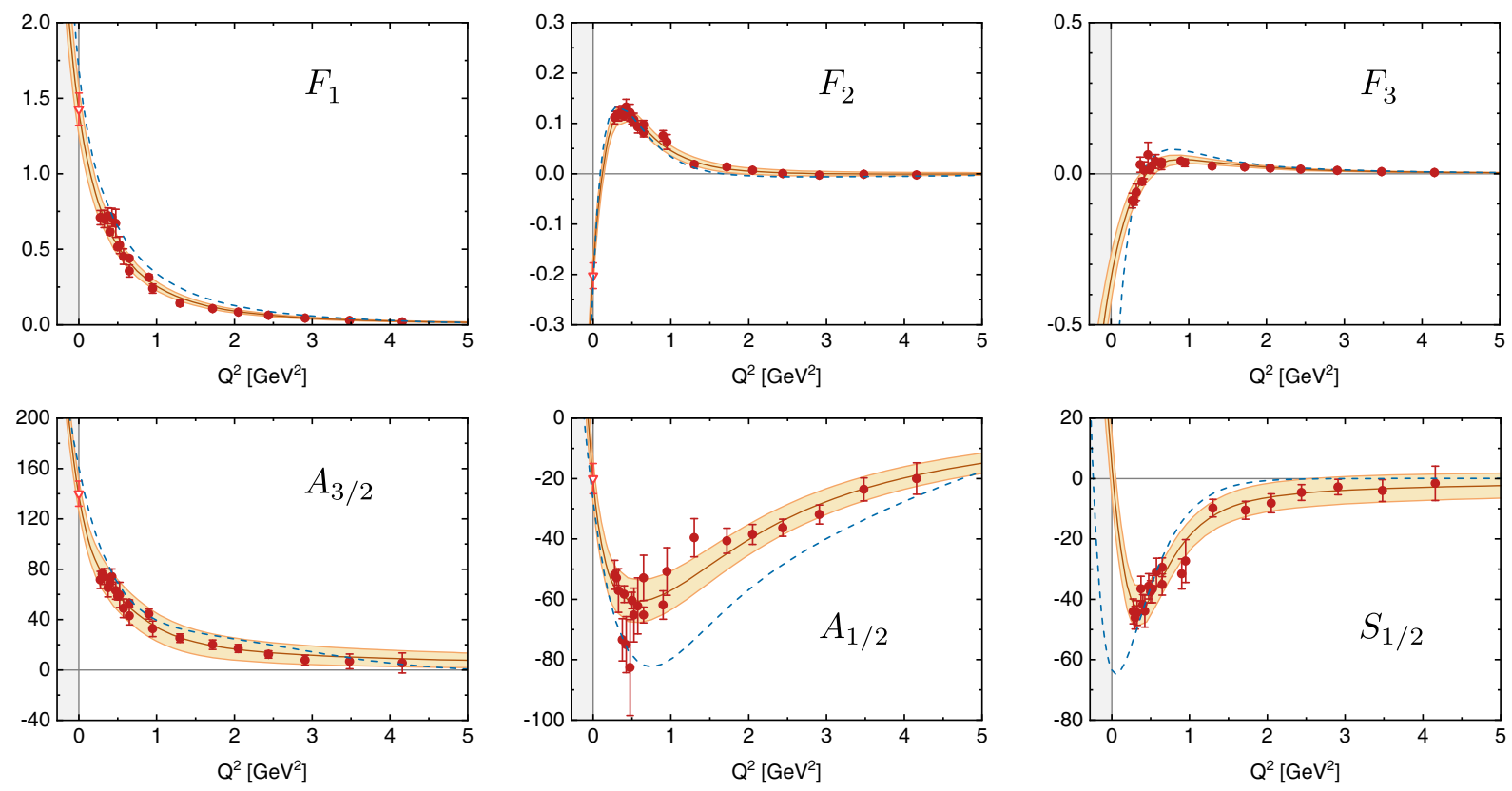

FIG. 14. Same as Fig. 12 but for $\gamma^{*} N \rightarrow N(1520)$. The data are from PDG [78] and CLAS/JLab [44,73,74].

Finally, given the asymptotic constraint $R_{\mathrm{EM}} \rightarrow 1$ this ratio must cross zero at some value $Q^{2}>0$. From Eq. (D20) the location of the zero in $R_{\mathrm{EM}}$ is

$$
Q^{2}\left(1+\frac{2 m_{R}}{m} \frac{F_{3}}{F_{2}}\right)=m_{R}^{2}-m^{2}
$$

In the absence of $F_{3}$ the zero crossing would happen early on, but because $F_{2}$ is negative the presence of $F_{3}$ pushes it to larger $Q^{2}$. The existing data do not show a clear trend in any direction but stay essentially constant. Note that the ratios in Fig. 12 are plotted in percent, so the constraint entails $R_{\mathrm{EM}} \rightarrow+100 \%$. The central value of our fit crosses zero at $Q^{2} \sim 20 \mathrm{GeV}^{2}$ but within the uncertainty band any other value above $Q^{2} \sim 7 \mathrm{GeV}^{2}$ is equally possible. Similarly, the large- $Q^{2}$ behavior for the ratio $R_{\mathrm{SM}}$ also depends on $F_{3}$ :

$$
R_{\mathrm{SM}} \underset{F_{1} \rightarrow-F_{2}}{\stackrel{Q^{2} \rightarrow \infty}{\rightarrow}}-1+\frac{Q^{2}}{2 m m_{R}} \frac{F_{3}}{F_{1}} .
$$

$\Delta(1700)$ : The ground state in the $(I) J^{P}=(3 / 2) 3 / 2^{-}$ channel is again an example where data are scarce. In this case the data points are compatible with all form factors being monotonous and negative, although this does not reproduce the large- $Q^{2}$ constraint $F_{1} \simeq-F_{2}$. In accordance with our previous strategy we fit the $H_{x}$ form factor data by constants.

$N(1520)$ : The transition form factors of the $(I) J^{P}=$ $(1 / 2) 3 / 2^{-}$ground state in Fig. 14 display rather peculiar features. $F_{1}$ is clearly monotonous but $F_{2}$ and $F_{3}$ are not. $F_{3}$ crosses zero at low $Q^{2}$, although the situation is somewhat reminiscent of the $\Delta(1232) . F_{2}$, on the other hand, appears to have two zero crossings: one at very low $Q^{2}$ between the PDG value and the CLAS data, and another one around $Q^{2} \sim 3 \mathrm{GeV}^{2}$ (although within the error bars the data are still compatible with zero). A negative value at large $Q^{2}$ would indeed be consistent with the constraint $F_{2} \simeq-F_{1}$. Still, this hints towards an interesting structure in the timelike region: $F_{2}$ is small compared to $F_{1}$, so potential meson-cloud effects induced by the cut structure could be magnified. Given the amount and precision of the data for this resonance, it is also the only example among all states considered where such features are clearly visible in a form factor. For these reasons we also include the parameter $a_{1}$ in our fit to achieve good parametrizations for $F_{2}$ and $F_{3}$. Significant meson-cloud contributions for the transverse amplitude $A_{3 / 2}$, which is generally underestimated by quark models [104,122-125], have also been suggested by dynamical coupled-channel calculations $[44,110]$.

$N(1720)$ : The ground state in the $(I) J^{P}=(1 / 2) 3 / 2^{+}$ channel is presently the highest-lying resonance where electroproduction data exist. In Fig. 15 one can see that here it is not even possible to pin down the sign for any form factor: all three $F_{i}$ contain data with both positive and negative signs, even among the three CLAS points. This clearly calls for more measurements in the future. One should also note that another $N(1720)$ state with the same quantum numbers was recently proposed to explain the $\gamma^{*} N \rightarrow \pi \pi N$ data $[43,45]$. We follow our previous strategy and fit the $H_{x}$ form factor data by constants; the resulting uncertainty bands provide at least a rough estimate for the magnitude of each form factor. 

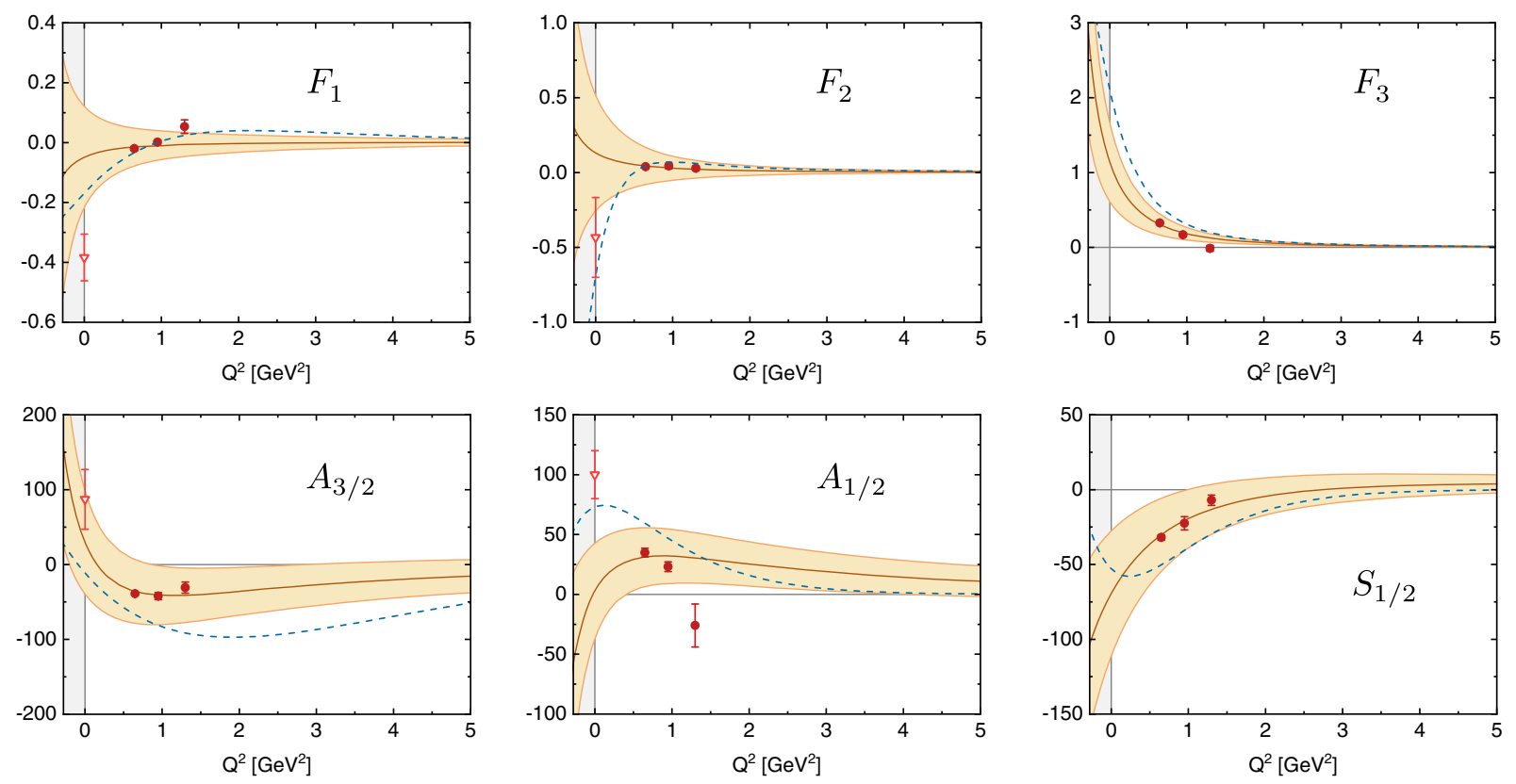

FIG. 15. Same as Fig. 12 but for $\gamma^{*} N \rightarrow N(1720)$. The data are from PDG [78] and CLAS/JLab [85].

\section{Discussion}

We constructed parametrizations for the $\gamma^{*} N \rightarrow R$ transition form factors based on analytic properties. Instead of fitting the data for the helicity amplitudes we directly fitted the constraint-free form factors. In the majority of cases these show a monotonous behavior which is well described by simple parametrizations. For the cases where data coverage is still poor we did not attempt to achieve a pointwise description but rather employed qualitative fits. In any case, the resulting helicity amplitudes automatically satisfy the kinematic constraints e.g., at the pseudothreshold, which can lead to significant deviations from the MAID parametrizations especially near the photon point.

The fits can be improved when more data become available. The $Q^{2}$-dependence of several transition form factors is still poorly known, especially at low $Q^{2}$ : even the best known resonances such as the $N(1440)$, $N(1520)$ and $N(1535)$ do not have any data below $Q^{2} \lesssim 0.3 \mathrm{GeV}^{2}$. This is particularly relevant for the form factors $F_{1}\left(Q^{2}\right)$ for $J=1 / 2$ states and $F_{3}\left(Q^{2}\right)$ for $J=$ $3 / 2$ states, which cannot be extracted at the photon point because $S_{1 / 2}(0)$ cannot be measured directly. It is then mandatory to expand the databases in this region to pin down the trend of the transition form factors near $Q^{2}=0$, which is also the relevant region for Compton scattering.

\section{COMPTON FORM FACTORS}

We now have everything in place to work out the nucleon resonance contributions to the CFFs. In practice we set up the resonance terms in Eqs. (5.1) and (6.1) in a specific Lorentz frame, Eqs. (A8)-(A9), and extract the CFFs by matrix inversion. For the $J^{P}=1 / 2^{ \pm}$resonances we employ the off-shell transition vertex (5.11) and for the $J^{P}=3 / 2^{ \pm}$ cases we use Eq. (6.24), together with our parametrizations for the transition form factors.

Fig. 16 shows the resulting CFFs inside the TPE cone. The bands include the dependence on all four variables $\eta_{+}, \eta_{-}, \omega$ and $\lambda$ as well as the uncertainty bands from our form factor parametrizations. In particular, we plot the residues $\hat{c}_{i}$ at the $s$ and $u$-channel poles defined by

$$
c_{i}\left(\eta_{+}, \eta_{-}, \omega, \lambda\right)=\delta^{2} \frac{\hat{c}_{i}\left(\eta_{+}, \eta_{-}, \omega, \lambda\right)}{\left(\eta_{-}+\delta\right)^{2}-4 \lambda^{2}},
$$

so that the poles do not appear in the plots but the static values at $\eta_{+}=\eta_{-}=\lambda=\omega=0$ can be read off directly.

One can see that the $\hat{c}_{i}$ typically fall into relatively thin bands. In the few cases where the bands are broader this is mainly due to the uncertainties from the form factors. Obviously this would not have been possible with a nonminimal basis: if some of the CFFs had kinematic singularities inside the cone or on its boundary, the spread of the bands would become infinite. Instead, the bands are narrow so that the dependence on four variables effectively reduces to a one-dimensional dependence on $\eta_{+}$. This is very helpful because instead of facing the need for studying many different kinematic slices the essential information is already encoded in a single variable.

Concerning the individual resonances, the $\Delta(1232)$ clearly provides the largest contribution to most CFFs. 

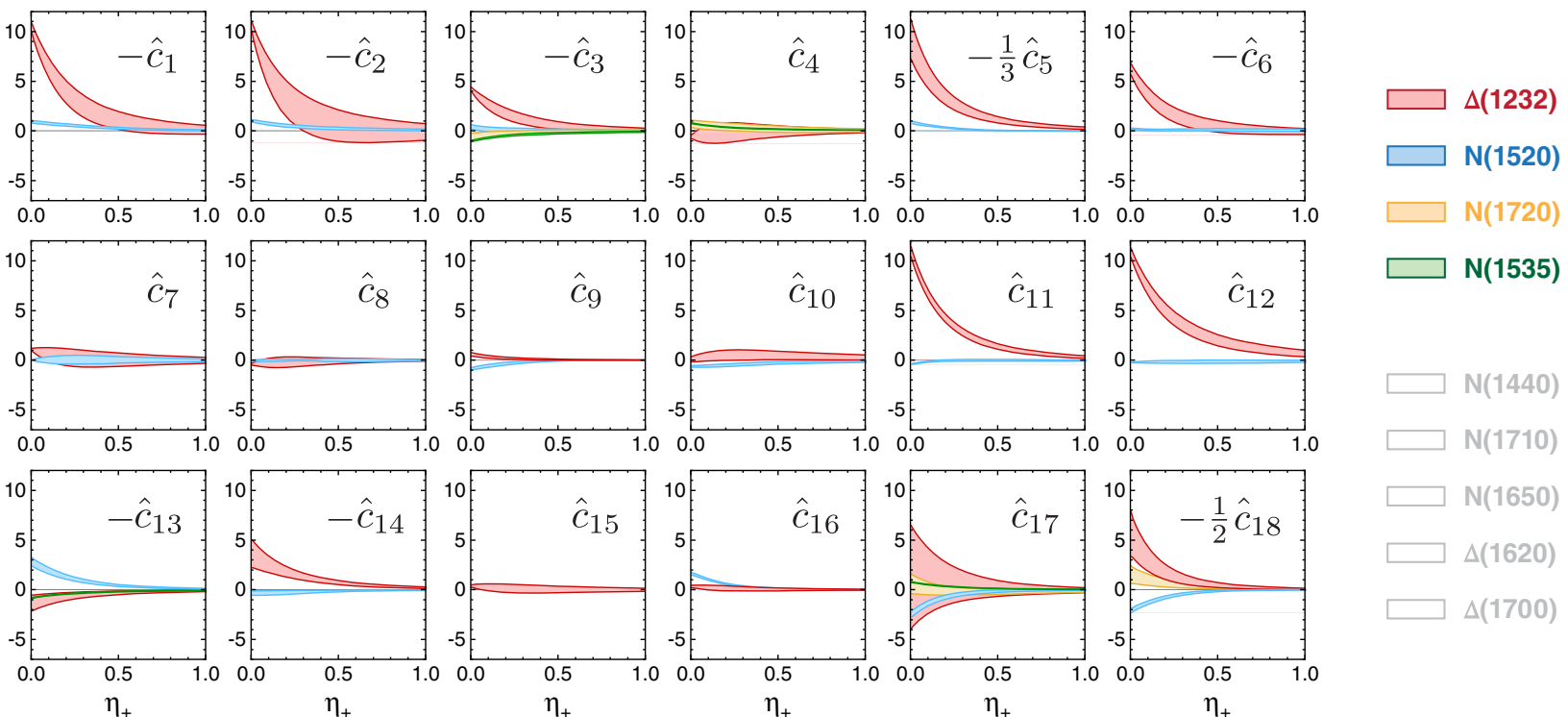

FIG. 16. Residues of the Compton form factors inside the TPE cone, plotted as functions of $\eta_{+}$. The bands contain the variation inside the cone as well as the uncertainties from the form factor parametrizations. The contributions from states other than the $\Delta(1232)$ and $N(1520)$ are only shown for $\hat{c}_{3,4,13,17,18}$ where they are visibly different from zero.

The higher-lying states usually only have little impact. The biggest subleading contributions come from the $N(1520)$ and $N(1720)$, which all carry spin $3 / 2$ as well, whereas the $J=1 / 2$ resonances such as the Roper resonance or the $N(1535)$ are almost negligible.

Table XIII collects the CFFs $c_{1}, c_{2}, c_{6}, c_{10}, c_{11}$ and $c_{12}$ in the static limit where all kinematic variables vanish. From the results in Tables VI and IX one extracts the following relations for $J^{P}=1 / 2^{ \pm}$states in that limit:

$$
\left[\begin{array}{c}
c_{1} \\
c_{2} \\
c_{6} \\
c_{10} \\
c_{11} \\
c_{12}
\end{array}\right]=\frac{F_{2}^{2}}{\delta^{2}}\left[\begin{array}{c}
\mp \delta \\
-\delta \delta_{ \pm} \\
\delta \delta_{\mp} \\
\pm \frac{1}{2} \delta \\
\mp 1 \\
-\delta_{ \pm}
\end{array}\right],
$$

TABLE XIII. Resonance contributions to the nucleon's scalar and spin polarizabilities. The experimental values of $\alpha$ and $\beta$ are from the PDG [78] and those for the spin polarizabilities from Refs. [126-128]. For the resonance masses that enter in Eqs. (8.2)-(8.3) we use the PDG 2016 estimates for the real parts of the pole positions [78]. The CFFs are dimensionless, $\alpha$ and $\beta$ carry units of $10^{-4}$ fm ${ }^{3}$, and the spin polarizabilities are given in $10^{-4} \mathrm{fm}^{4}$. We do not display the numbers if both their absolute values and uncertainties are smaller than 0.05 in the respective units.

\begin{tabular}{|c|c|c|c|c|c|c|c|c|c|c|}
\hline & Exp. & $N(1440)$ & $N(1710)$ & $N(1535)$ & $N(1650)$ & $\Delta(1620)$ & $\Delta(1232)$ & $\Delta(1700)$ & $N(1520)$ & $N(1720)$ \\
\hline$-c_{1}$ & 20.2(4) & $0.2(0)$ & $0.1(0)$ & $-0.3(1)$ & & & $10.6(8)$ & $-0.1(2)$ & $0.9(2)$ & $0.0(1)$ \\
\hline$-c_{2}$ & $3.7(6)$ & $0.2(0)$ & $0.1(1)$ & $0.1(0)$ & & & $10.8(8)$ & $0.0(1)$ & $1.0(2)$ & \\
\hline$-c_{6}$ & $27.8(4.1)$ & & & $-0.4(1)$ & $-0.1(0)$ & & $6.3(9)$ & $0.0(1)$ & $0.3(1)$ & $0.0(1)$ \\
\hline$c_{10}$ & $9.0(8.6)$ & $0.1(0)$ & & $-0.1(0)$ & & & $0.1(4)$ & $0.1(1)$ & $-0.6(1)$ & \\
\hline$c_{11}$ & $3.3(6.7)$ & $-0.2(0)$ & & $0.2(0)$ & & & $11.1(8)$ & & $-0.4(1)$ & \\
\hline$c_{12}$ & $8.6(5.1)$ & $-0.2(0)$ & & $-0.1(0)$ & & & $11.0(8)$ & $0.1(1)$ & $-0.2(0)$ & \\
\hline$\alpha$ & $11.2(4)$ & & & $-0.3(0)$ & & & $-0.1(0)$ & $-0.1(1)$ & $-0.1(0)$ & \\
\hline$\beta$ & $2.5(4)$ & $0.1(0)$ & $0.1(0)$ & $0.1(0)$ & & & 7.3(6) & $0.0(1)$ & $0.7(2)$ & \\
\hline$\gamma_{E 1 E 1}$ & $-3.5(1.2)$ & & & $0.1(0)$ & & & $-0.4(0)$ & & $-0.1(0)$ & \\
\hline$\gamma_{M 1 M 1}$ & $3.2(0.9)$ & $-0.1(0)$ & & & & & $3.6(3)$ & & $0.1(0)$ & \\
\hline$\gamma_{E 1 M 2}$ & $-0.7(1.2)$ & & & & & & $-0.4(0)$ & & $-0.1(0)$ & \\
\hline$\gamma_{M 1 E 2}$ & $2.0(0.3)$ & & & & & & $0.4(1)$ & & & \\
\hline$\gamma_{0}$ & $-0.9(0.1)$ & & & $-0.1(0)$ & & & $-3.2(2)$ & & $0.1(0)$ & \\
\hline$\gamma_{\pi}$ & $8.0(1.8)$ & $-0.1(0)$ & & $-0.2(0)$ & & & 4.9(4) & & $0.3(0)$ & \\
\hline
\end{tabular}


whereas for $J^{P}=3 / 2^{ \pm}$resonances one obtains

$$
\left[\begin{array}{c}
c_{1} \\
c_{2} \\
c_{6} \\
c_{10} \\
c_{11} \\
c_{12}
\end{array}\right]=\frac{2}{3 \delta^{2}}\left[\begin{array}{c}
-4 \delta G_{2} \\
-4 \delta \delta_{ \pm} F_{1}^{2} \\
2 \delta F_{2}\left(3 \delta_{ \pm} F_{1}-\delta_{\mp} F_{2}\right) \\
-\delta\left(G_{2} \pm 3 F_{1} F_{2}\right) \\
\pm 2\left(G_{1}-\delta F_{1} F_{2}\right) \\
2 \delta_{ \pm}\left(G_{1}+(r \mp 2) \delta_{\mp} F_{1} F_{2}\right)
\end{array}\right] .
$$

We abbreviated $G_{1}=\delta_{ \pm}^{2} F_{1}^{2}+\delta_{\mp}^{2} F_{2}^{2}$ and $G_{2}=\delta_{ \pm} F_{1}^{2}-$ $\delta_{\mp} F_{2}^{2}$, and the $F_{i} \equiv F_{i}\left(Q^{2}=0\right)$ denote the static values of the transition form factors. With our parametrizations they are fully specified by the parameters $a_{0} \pm e_{0}$. For a spin- $1 / 2$ resonance only the form factor $F_{2}(0)$ contributes and for a spin-3/2 state only $F_{1}(0)$ and $F_{2}(0)$ survive. These CFFs are related to the scalar and spin polarizabilities through Eqs. (2.31)-(2.33); for example the scalar polarizabilities $\alpha$ and $\beta$ become

$$
\left[\begin{array}{c}
\alpha \\
\beta
\end{array}\right]=\frac{\alpha_{\mathrm{em}}}{m^{3}}\left\{\begin{array}{cc}
\frac{F_{2}^{2}}{\delta}\left[\begin{array}{c}
-\delta_{\mp} \\
\delta_{ \pm}
\end{array}\right] & \ldots J^{P}=\frac{1}{2}^{ \pm}, \\
\frac{8}{3 \delta}\left[\begin{array}{c}
-\delta_{\mp} F_{2}^{2} \\
\delta_{ \pm} F_{1}^{2}
\end{array}\right] & \ldots J^{P}=\frac{3}{2}{ }^{ \pm} .
\end{array}\right.
$$

Those CFFs in Fig. 16 that are more sensitive to the higher-lying resonances do not contribute to the polarizabilities, so that also here mainly the $\Delta(1232)$ is relevant. The largest subleading effects come from the $N(1520)$ and $N(1535)$ but they are very small. That the $\Delta$ plays an important role is of course well known, and chiral effective field theory and dispersive approaches provide a more quantitative description than the simple tree-level expressions that we collect here [1-5]. For example, pion loops reduce the large $\Delta$ contribution to the magnetic polarizability $\beta$ and, as a result of this cancellation, $\beta$ is small compared to the electric polarizability $\alpha$. The sum $\alpha+\beta$ is proportional to $c_{1}$ and constrained by the Baldin sum rule [129]. Indeed, Table XIII shows that none of the resonances contributes anything substantial to $\alpha$. What is noteworthy is the $N(1520)$ contribution to $\beta$, which is about a quarter of the size of its PDG value.

For the spin polarizabilities the situation is less well established. Fig. 17 contrasts experimental results from A2/MAMI [126] with predictions from dispersion theory and chiral perturbation theory. Instead of the spin polarizabilities, we plot the CFFs directly because their leading ChPT values from Eq. (2.34) are simple: $c_{12}$ vanishes and after removing the pion pole in $c_{6}$ the remaining ones are identical up to signs. The various chiral approaches display sizeable uncertainties. The difficulty arises from the $\Delta$ resonance, which has a large effect and should be incorporated in the description. In Fig. 17 one can see that even by
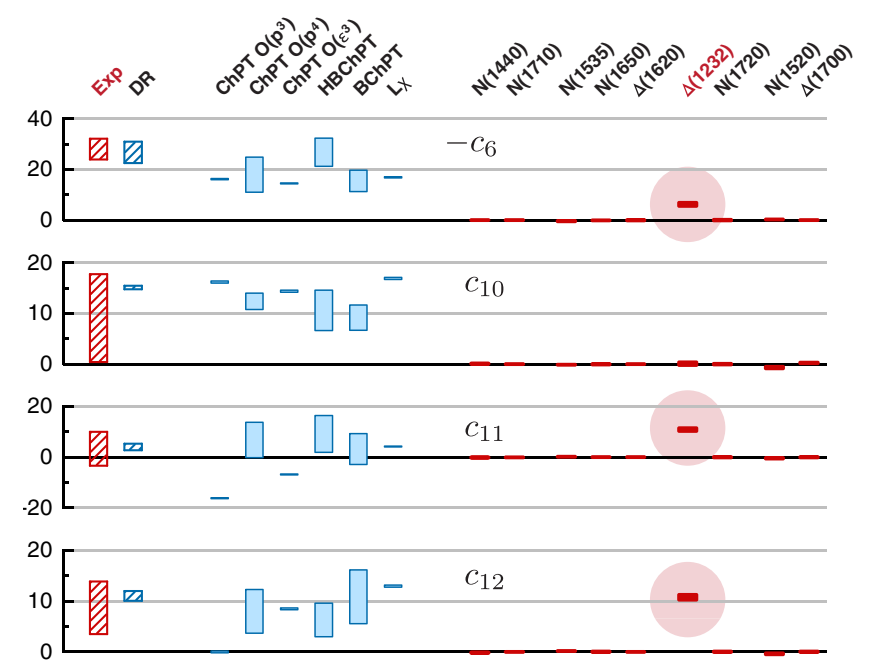

FIG. 17. Resonance contributions to the spin polarizabilities encoded in the static values of $c_{6}, c_{10}, c_{11}$ and $c_{12}$. We compare to experiment [126], dispersion theory [1,130,131], leading-order ChPT [54], and higher-order chiral approaches [132-137]; see Refs. [1,5] for compilations. For $c_{6}$ the pion pole contribution from Eq. (2.34) has been excluded.

simply adding together the $\Delta$ and leading-order ChPT results one obtains values that are roughly compatible with experiment and dispersion theory. On the other hand, it is reassuring that it is practically only the $\Delta$ resonance that has an impact on spin polarizabilities because all other resonances are negligible.

Returning to the CFFs in general kinematics, the practical result is that the same 18 CFFs describe the Compton scattering process in general. If one had complete knowledge of the Compton amplitude that information could be condensed in the 18 panels of Fig. 16 as well. For Compton scattering on a pointlike scalar particle only $c_{1}$ and $c_{2}$ survive; for a nonpointlike scalar the first five $c_{i}$ contribute; the scalar $t$-channel poles can only appear in $c_{2}$ and $c_{3}$; pseudoscalar poles can only appear in $c_{6}$; the nucleon Born term contributes to eight CFFs in Fig. 5; in RCS the six CFFs discussed above remain; in VCS the twelve CFFs from Eq. (2.37) and in the forward limit the four combinations in Eq. (2.41) survive.

The question is then whether the observation from Fig. 16 also holds in other kinematic limits, i.e., whether the momentum dependencies in the variables $\eta_{-}, \omega$ and $\lambda$ are generally weak. In general the answer depends on the singularity structure: viewed as analytic functions, the CFFs are determined by their physical singularities. Our present situation is of course rather special because we merely add up tree-level resonances. The formulas for the resonance terms contain $s$ and $u$-channel nucleon resonance poles together with timelike poles in $Q^{2}$ and $Q^{\prime 2}$ from the transition form factors.

Consider e.g., the situation in VCS, which is illustrated in Fig. 18. At fixed $\eta_{+}$, the TPE cone becomes a circle and 

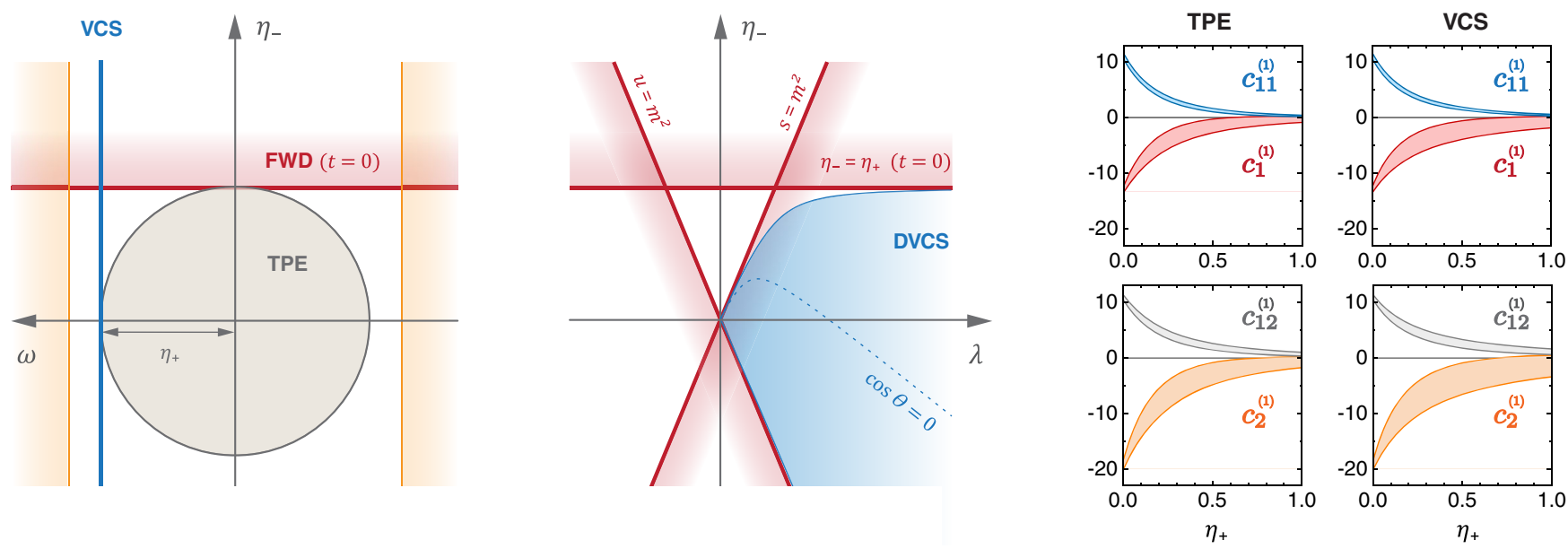

FIG. 18. Left: Singularity structure of the Compton scattering amplitude at fixed $\eta_{+}$in the $\left\{\omega, \eta_{-}\right\}$and $\left\{\lambda, \eta_{-}\right\}$planes; see text for a discussion. Right: Compton form factor residues for the $\Delta(1232)$ resonance inside the TPE and VCS regions.

the VCS and forward planes become the lines with $\omega=\eta_{+}$ and $\eta_{-}=\eta_{+}$, respectively. In the limit $\eta_{+} \rightarrow 0$, the circle shrinks to a point and VCS collapses into RCS. The vector-meson poles in the transition form factors appear at timelike photon virtualities, which correspond to $\omega= \pm\left(\eta_{+}+m_{\rho}^{2} / m^{2}\right)$ as indicated by the vertical (orange) bands. They are symmetric in $\omega$ and the CFFs depend on $\omega$ only quadratically, so it is clear that a multipole falloff in the form factors cannot induce an overly strong $\omega$ dependence in the interior $|\omega| \lesssim \eta_{+}$but mainly affects the momentum dependence in $\eta_{+}$.

Including also the remaining variable $\lambda$ (again at fixed $\eta_{+}$) yields the Mandelstam plane in $\lambda$ and $\eta_{-}$. This is where the resonance poles at $\eta_{-}= \pm 2 \lambda$ become visible, which are illustrated by the diagonal (red) bands in Fig. 18. The physical VCS region is the shaded (blue) area with $|\cos \theta|<1$, where $\theta$ is the CM scattering angle. In RCS it would be the domain enclosed between $\eta_{-}=0$ and $\eta_{-}=1-\sqrt{1+4 \lambda^{2}}$; for increasing $\eta_{+}$the line $t=0$ moves upwards and "drags" the physical region with it. DVCS is the domain of large $\lambda$ and moderate $t$.

Clearly, the dependence in $\lambda$ cannot be weak because this is where the resonance bumps appear. Recalling the discussion around Eq. (4.21), the $\lambda$ dependence of the resonance terms is trivial and can be removed by splitting the CFFs into non-resonant and resonant parts,

$$
c_{i}\left(\eta_{+}, \eta_{-}, \omega, \lambda\right)=c_{i}^{(0)}\left(\eta_{+}, \eta_{-}, \omega\right)+\frac{\delta^{2} c_{i}^{(1)}\left(\eta_{+}, \eta_{-}, \omega\right)}{\left(\eta_{-}+\delta\right)^{2}-4 \lambda^{2}}
$$

where $c_{i}^{(0)}$ and $c_{i}^{(1)}$ no longer depend on $\lambda$. At fixed $\eta_{+}$and with the $\omega$ dependence being weak, the remaining question is how strong their variation in $\eta_{-}$is.

The right panels in Fig. 18 compare some of the $c_{i}^{(1)}$ inside the TPE cone and in VCS. In the VCS case we limit the range of $\eta_{-}$to $-\eta_{+}<\eta_{-}<\eta_{+}$with the same extent as the cone, whereas inside the cone the functions vary over the full $\eta_{-}$and $\omega$ range. The bands do not change substantially, which generally also holds for the remaining CFFs and means that the VCS region is still sufficiently close to the cone. Thus, in principle one could predict the functional dependence of the CFFs in VCS from their knowledge, e.g., near the symmetric limit where all variables except $\eta_{+}$vanish.

While these observations are particular to the case of resonances, they can be useful in more general situations. From the viewpoint of analyticity, the fact that the CFFs (and therefore structure functions) depend on $\lambda$ at all is tied to the $s$ - and $u$-channel resonance structure, which points to the idea of quark-hadron duality [138-140]. On the other hand, in the microscopic decomposition of Compton scattering the nucleon resonance structure is produced by different quarkgluon topologies than the handbag and $t$-channel diagrams [27]. Without an underlying mechanism to generate singularities in $\lambda$, the momentum dependence in that variable would disappear; unless it creates unphysical singularities, but those must ultimately cancel with other parts of the amplitude. Such calculations are typically easier to perform inside the TPE cone (the "Euclidean region") where one can avoid timelike singularities in the underlying correlation functions like the quark propagator; cf. Refs. [141,142] for studies of the pion transition form factor. Thus it may be possible to analytically continue results inside the cone, where $\lambda$ is imaginary, also to the domain of large and real $\lambda$ which is accessible in DVCS.

Finally, the discussion in terms of Lorentz-invariant and constraint-free CFFs can be useful for amplitude analyses of Compton scattering [143]. For illustration we plot in Fig. 19 the reconstructed Compton amplitude in RCS, in particular the imaginary parts of the six associated CFFs, inside the physical RCS region and between $-2<\eta_{-}<0$. We equipped the resonances with widths according to Eq. (2.16), where $m_{R}$ and $-\Gamma / 2$ are taken to be the real and 


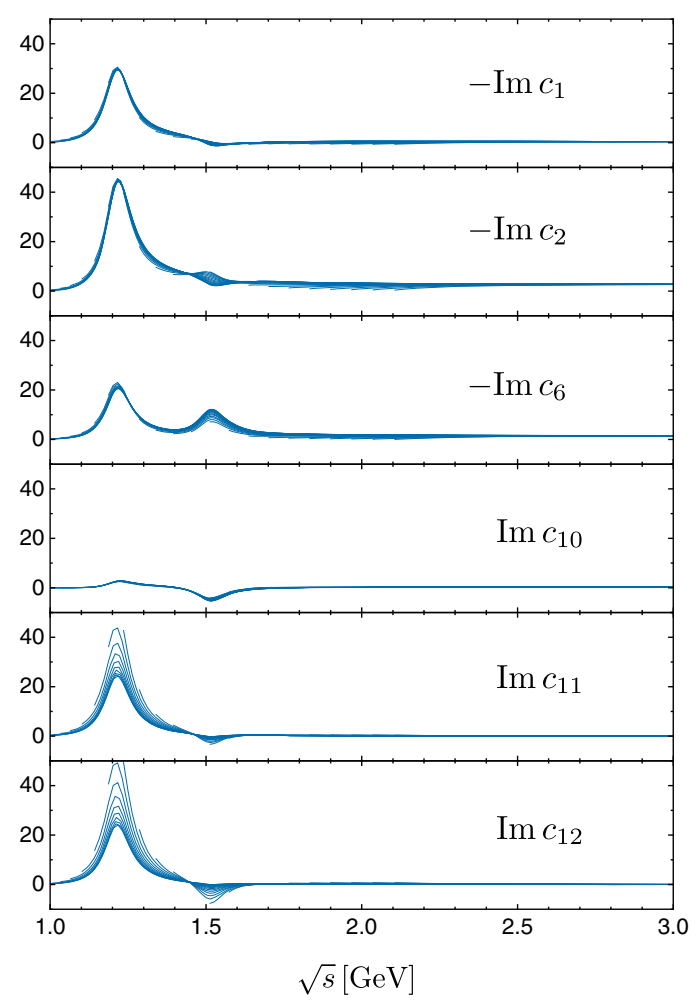

FIG. 19. Imaginary parts of the six Compton form factors in RCS, plotted in the physical $s$-channel region for different values of $\eta_{-}$between $-2<\eta_{-}<0$.

imaginary parts of the respective pole positions from the PDG [78]. Analogous plots can be drawn in the forward limit, where the imaginary parts are proportional to the nucleon's structure functions, or in VCS. The $\Delta(1232)$ clearly dominates, whereas other resonances such as the $N(1520)$ are enhanced in particular CFFs; and with Tables VI and IX one can form CFF combinations where resonances with specific $J^{P}$ drop out.

\section{SUMMARY AND OUTLOOK}

In this work we have detailed the tensor basis construction for Compton scattering on the nucleon, which implements the constraints of electromagnetic gauge invariance, crossing symmetry, and the absence of kinematic singularities. The resulting 18 Lorentz-invariant Compton form factors are free of kinematic constraints and describe the process in general kinematics.

As a practical application we worked out the Compton form factor contributions coming from intermediate $J^{P}=$ $1 / 2^{ \pm}$and $3 / 2^{ \pm}$nucleon resonances. We derived the general forms for their off-shell nucleon-to-resonance transition vertices according to electromagnetic and spin-3/2 gauge invariance. This automatically defines constraint-free onshell transition form factors, for which we constructed fits using the available experimental data. We find that apart from the $\Delta(1232)$ the resonance contributions to the scalar and spin polarizabilites are very small, although the $N(1520)$ could play a role for the proton's magnetic polarizability.

Our study can be extended to calculate resonance contributions to two-photon exchange processes or baryons with higher spin. Moreover, since the tensor basis construction follows a general recipe it provides a template for other systems, such as the hadronic light-by-light scattering amplitude which enters in the standard model prediction for the muon anomalous magnetic moment.

\section{ACKNOWLEDGMENTS}

We acknowledge interactions with B. Bakker, C. S. Fischer, R. Gothe and V. Mokeev. This work was supported by the FCT Investigator Grant No. IF/00898/2015 and the Fundação de Amparo à Pesquisa do Estado de São Paulo (FAPESP) under Project No. 2017/02684-5 and Grant No. 2017/17020-BCO-JP.

\section{APPENDIX A: CONVENTIONS AND FORMULAS}

\section{Euclidean vs Minkowski conventions}

We use Euclidean conventions throughout this paper, but we took care in constructing the notation such that many identities are the same in Euclidean and Minkowski conventions. In the following we summarize the transcription rules from a Minkowski metric with signature $(+,-,-,-)$ to a Euclidean metric $(+,+,+,+)$; more relations can be found in Appendix A of Ref. [28].

The replacement rules for vectors $a^{\mu}$ and tensors $T^{\mu \nu}$ are given by

$$
a_{E}^{\mu}=\left[\begin{array}{c}
\boldsymbol{a} \\
i a_{0}
\end{array}\right], \quad T_{E}^{\mu \nu}=\left[\begin{array}{cc}
T^{i j} & i T^{i 0} \\
i T^{0 i} & -T^{00}
\end{array}\right],
$$

where ' $E$ ' stands for Euclidean and no subscript refers to the Minkowski quantity. As a consequence, the Lorentzinvariant scalar product of any two four-vectors differs by a minus sign from its Minkowski counterpart:

$$
a_{E} \cdot b_{E}=\sum_{k=1}^{4} a_{E}^{k} b_{E}^{k}=-a \cdot b
$$

Therefore, a vector is spacelike if $a^{2}>0$ and timelike if $a^{2}<0$. Because the metric is positive, the distinction between upper and lower indices disappears. To preserve the meaning of the slash $\not h=a^{0} \gamma^{0}-\boldsymbol{a} \cdot \boldsymbol{\gamma}$ we must also redefine the $\gamma$-matrices:

$$
i \gamma_{E}^{\mu}=\left[\begin{array}{c}
\gamma \\
i \gamma_{0}
\end{array}\right], \quad \gamma_{E}^{5}=\gamma^{5},
$$

so that 
TABLE XIV. Replacement rules for some typical quantities. For expressions with Lorentz indices, the right column defines their Euclidean version in the sense of Eqs. (A1) and (A3). Each additional Minkowski summation over Lorentz indices leads to a minus sign in Euclidean conventions.

\begin{tabular}{|c|c|}
\hline Minkowski & Euclidean \\
\hline$a \cdot b$ & $-a \cdot b$ \\
\hline$a^{\mu}$ & $a^{\mu}$ \\
\hline$\gamma^{\mu}$ & $i \gamma^{\mu}$ \\
\hline$\gamma_{5}$ & $\gamma_{5}$ \\
\hline$\not d$ & $-i \not h$ \\
\hline$g^{\mu \nu}$ & $-\delta^{\mu \nu}$ \\
\hline$a^{\mu} b^{\nu}$ & $a^{\mu} b^{\nu}$ \\
\hline$\left[\gamma^{\mu}, \gamma^{\nu}\right]$ & $-\left[\gamma^{\mu}, \gamma^{\nu}\right]$ \\
\hline$\left[\gamma^{\mu}, \not \subset\right]$ & {$\left[\gamma^{\mu}, \not \subset\right]$} \\
\hline $\begin{array}{l}{\left[\gamma^{\mu}, \gamma^{\nu}, \not d\right]} \\
{[\not, \not b]}\end{array}$ & $\begin{aligned} & i\left[\gamma^{\mu}, \gamma^{\nu}, \not d\right] \\
- & {[\not \phi, \not b] }\end{aligned}$ \\
\hline$\varepsilon^{\mu \nu \rho \alpha} a_{\alpha}$ & $i \varepsilon^{\mu \nu \rho \alpha} a^{\alpha}$ \\
\hline$\varepsilon^{\mu \nu \alpha \beta} a_{\alpha} b_{\beta}$ & $i \varepsilon^{\mu \nu \alpha \beta} a^{\alpha} b^{\beta}$ \\
\hline$\varepsilon^{\mu \alpha \beta \gamma} a_{\alpha} b_{\beta} c_{\gamma}$ & $i \varepsilon^{\mu \alpha \beta \gamma} a^{\alpha} b^{\beta} c^{\gamma}$ \\
\hline$\varepsilon^{\mu \nu \alpha \beta} a_{\alpha} \gamma_{\beta}$ & $-\varepsilon^{\mu \nu \alpha \beta} a^{\alpha} \gamma^{\beta}$ \\
\hline
\end{tabular}

$$
\not \phi_{E}=a_{E} \cdot \gamma_{E}=i \not d, \quad\left\{\gamma_{E}^{\mu}, \gamma_{E}^{\nu}\right\}=2 \delta^{\mu \nu} .
$$

Our sign convention for the Euclidean $\gamma$-matrices changes all signs in the Clifford algebra relation (A4) to be positive, and since this implies $\left(\gamma_{E}^{i}\right)^{2}=1$ for $i=1 \ldots 4$ we can choose them to be Hermitian: $\gamma_{E}^{\mu}=\left(\gamma_{E}^{\mu}\right)^{\dagger}$. For example, in the standard representation they read

$$
\gamma_{E}^{k}=\left[\begin{array}{cc}
0 & -i \tau_{k} \\
i \tau_{k} & 0
\end{array}\right], \quad \gamma_{E}^{4}=\left[\begin{array}{cc}
\mathbb{1} & 0 \\
0 & -\mathbb{1}
\end{array}\right], \quad \gamma^{5}=\left[\begin{array}{ll}
0 & \mathbb{1} \\
\mathbb{1} & 0
\end{array}\right]
$$

where the $\tau_{k}$ are the usual Pauli matrices. Also the generators of the Clifford algebra are then Hermitian:

$$
\sigma^{\mu \nu}=\frac{i}{2}\left[\gamma^{\mu}, \gamma^{\nu}\right] \Rightarrow \sigma_{E}^{\mu \nu}=-\frac{i}{2}\left[\gamma_{E}^{\mu}, \gamma_{E}^{\nu}\right]
$$

with $\left(\sigma_{E}^{\mu \nu}\right)^{\dagger}=\sigma_{E}^{\mu \nu}$.

The resulting replacement rules for some typical quantities are collected in Table XIV. For expressions involving the $\varepsilon$-tensor the situation is slightly more complicated but follows from the same principles: the spatial parts of Lorentz tensors are identical in Minkowski and Euclidean conventions, so this must also hold for $\varepsilon^{\mu \nu \alpha \beta} a_{\alpha} b_{\beta}$. In Euclidean space the $\varepsilon$-tensor is defined by $\varepsilon_{1234}=\varepsilon^{1234}=1$, whereas in Minkowski conventions one has $\varepsilon_{0123}=-\varepsilon^{0123}=1$, i.e., the spatial components switch sign when lowering or raising indices. Denoting spatial indices by $i, j, k$ and summing over $k$, one has

$$
\begin{aligned}
\varepsilon^{i j \alpha \beta} a_{\alpha} b_{\beta} & =\varepsilon^{i j k 0}\left(a_{k} b_{0}-a_{0} b_{k}\right) \\
& =-\varepsilon^{i j k 0}\left(a^{k} b^{0}-a^{0} b^{k}\right) \\
& =i \varepsilon^{i j k 4}\left(a^{k} b^{4}-a^{4} b^{k}\right)_{E} \\
& =\left(i \varepsilon^{i j \alpha \beta} a^{\alpha} b^{\beta}\right)_{E},
\end{aligned}
$$

because $\varepsilon^{1234}=1=\varepsilon^{1230}$ and $a^{0}=-i a_{E}^{4}$. Repeating this for rank-1 and rank-3 tensors results in the analogous identities in Table XIV [which would also follow from Eq. (A16) below)].

With these rules it is straightforward to transform Euclidean tensors, such as e.g., those in Table XV, to Minkowski space. To further facilitate the transcription, we have introduced the variables $\eta_{+}, \eta_{-}, \lambda$ and $\omega$ in the main text, Eq. (2.6), because they allow for a common definition in Euclidean and Minkowski space. For example, with $q_{E}^{\mu}=Q^{\mu}$ :

$$
\eta_{+}=\frac{Q^{2}+Q^{\prime 2}}{2 m^{2}}=-\frac{q^{2}+q^{\prime 2}}{2 m^{2}},
$$

and so on for the remaining variables. Once the momentum variables and Lorentz tensors are given appropriate names, all Lorentz-covariant and Lorentz-invariant relations between them are the same in Euclidean and Minkowski conventions.

The advantage of the Euclidean metric is that one can perform numerical calculations directly in a given frame, with explicit $\gamma$ matrices and without the need for inserting the metric tensor in each summation. A practical Lorentz frame for the momenta $p, \Sigma$ and $\Delta$ defined in Eq. (2.7) is [27]

$$
\begin{aligned}
& \frac{\Delta^{\mu}}{m}=2 \sqrt{t}\left[\begin{array}{l}
0 \\
0 \\
0 \\
1
\end{array}\right], \quad \frac{\Sigma^{\mu}}{m}=\sqrt{\sigma}\left[\begin{array}{c}
0 \\
0 \\
\sqrt{1-Z^{2}} \\
Z
\end{array}\right], \\
& \frac{p^{\mu}}{m}=i \sqrt{1+t}\left[\begin{array}{c}
0 \\
\sqrt{1-Y^{2}} \\
Y \\
0
\end{array}\right],
\end{aligned}
$$

where $t, \sigma, Z$ and $Y$ are defined in Eqs. (2.8)-(2.10). Inside the TPE cone, the angular variables $Z$ and $Y$ fill the interval $[-1,1]$ whereas in its exterior they can become complex. In the VCS limit it is more convenient to use the frame where $p^{\mu}=i m \sqrt{1+t}[0,0,0,1]$ and

$$
\frac{Q^{\prime \mu}}{m}=\alpha\left[\begin{array}{l}
0 \\
0 \\
1 \\
i
\end{array}\right], \quad \frac{Q^{\mu}}{m}=\left[\begin{array}{c}
0 \\
\sqrt{4 t-\beta^{2}} \\
\alpha+\beta \\
i \alpha
\end{array}\right],
$$


TABLE XV. Elementary tensors for the nucleon Compton scattering amplitude. We suppressed the Lorentz indices for $K_{i}^{\mu \nu}$ and $T_{i}^{\mu \nu}$ to avoid clutter. The $K_{i}$ are dimensionless and invariant under photon crossing and charge conjugation. The $T_{i}$ are the same as in Eq. (8) of Ref. [29]. $n$ counts the powers of photon momenta; $\lambda$ contributes one power and $\omega$ two.

\begin{tabular}{|c|c|c|c|}
\hline$n$ & Basis element & $n$ & Basis element \\
\hline 0 & $K_{1}=-T_{1}=\delta^{\mu \nu}$ & 2 & $i m K_{11}=-i \lambda T_{11}=\lambda \delta^{\mu \nu} \nsucceq$ \\
\hline 2 & $m^{2} K_{2}=T_{2}=Q^{\prime \mu} Q^{\nu}$ & 4 & $i m^{3} K_{12}=i \lambda T_{12}=\lambda Q^{\prime \mu} Q^{\nu} \nsubseteq$ \\
\hline 2 & $m^{2} K_{3}=T_{3}=Q^{\mu} Q^{\prime \nu}$ & 4 & $i m^{3} K_{13}=i \lambda T_{13}=\lambda Q^{\mu} Q^{\prime \nu} \not$ \\
\hline 2 & $m^{2} K_{4}=T_{4}=Q^{\mu} Q^{\nu}+Q^{\prime \mu} Q^{\prime \nu}$ & 4 & $i m^{3} K_{14}=i \lambda T_{14}=\lambda\left(Q^{\mu} Q^{\nu}+Q^{\prime \mu} Q^{\prime \nu}\right) \not$ \\
\hline 4 & $m^{2} K_{5}=\omega T_{5}=\omega\left(Q^{\mu} Q^{\nu}-Q^{\prime \mu} Q^{\prime \nu}\right)$ & 6 & $i m^{3} K_{15}=i \lambda \omega T_{15}=\lambda \omega\left(Q^{\mu} Q^{\nu}-Q^{\prime \mu} Q^{\prime \nu}\right) \Psi$ \\
\hline 0 & $m^{2} K_{6}=T_{6}=p^{\mu} p^{\nu}$ & 2 & $i m^{3} K_{16}=i \lambda T_{16}=\lambda p^{\mu} p^{\nu} \Psi$ \\
\hline 2 & $m^{2} K_{7}=\lambda T_{7}=\lambda\left(p^{\mu} Q^{\prime \nu}+Q^{\mu} p^{\nu}\right)$ & 2 & $i m^{3} K_{17}=i T_{17}=\left(p^{\mu} Q^{\prime \nu}+Q^{\mu} p^{\nu}\right) \leftleftarrows$ \\
\hline 4 & $m^{2} K_{8}=-\lambda \omega T_{8}=\lambda \omega\left(p^{\mu} Q^{\prime \nu}-Q^{\mu} p^{\nu}\right)$ & 4 & $i m^{3} K_{18}=-i \omega T_{18}=\omega\left(p^{\mu} Q^{\prime \nu}-Q^{\mu} p^{\nu}\right) 叉$ \\
\hline 2 & $m^{2} K_{9}=\lambda T_{9}=\lambda\left(p^{\mu} Q^{\nu}+Q^{\prime \mu} p^{\nu}\right)$ & 2 & $i m^{3} K_{19}=i T_{19}=\left(p^{\mu} Q^{\nu}+Q^{\prime \mu} p^{\nu}\right) \nsubseteq$ \\
\hline 4 & $m^{2} K_{10}=-\lambda \omega T_{10}=\lambda \omega\left(p^{\mu} Q^{\nu}-Q^{\prime \mu} p^{\nu}\right)$ & 4 & $i m^{3} K_{20}=-i \omega T_{20}=\omega\left(p^{\mu} Q^{\nu}-Q^{\prime \mu} p^{\nu}\right) \leftleftarrows$ \\
\hline 3 & $m^{2} K_{27}=\omega T_{27}=\omega\left[p^{\mu} \gamma^{\nu}+\gamma^{\mu} p^{\nu}, \not \grave{L}\right]$ & 0 & $i m K_{21}=-i T_{21}=p^{\mu} \gamma^{\nu}+\gamma^{\mu} p^{\nu}$ \\
\hline 1 & $m^{2} K_{28}=-T_{28}=\left[p^{\mu} \gamma^{\nu}-\gamma^{\mu} p^{\nu}, \not{Z}\right]$ & 2 & $i m K_{22}=i \omega T_{22}=\omega\left(p^{\mu} \gamma^{\nu}-\gamma^{\mu} p^{\nu}\right)$ \\
\hline 5 & $m^{2} K_{29}=\lambda \omega T_{29}=\lambda \omega\left[Q^{\prime \mu} \gamma^{\nu}+\gamma^{\mu} Q^{\nu}, \not{Z}\right]$ & 2 & $i m K_{23}=-i \lambda T_{23}=\lambda\left(Q^{\prime \mu} \gamma^{\nu}+\gamma^{\mu} Q^{\nu}\right)$ \\
\hline 3 & $m^{2} K_{30}=-\lambda T_{30}=\lambda\left[Q^{\prime \mu} \gamma^{\nu}-\gamma^{\mu} Q^{\nu}, \not{Z}\right]$ & 4 & $i m K_{24}=i \lambda \omega T_{24}=\lambda \omega\left(Q^{\prime \mu} \gamma^{\nu}-\gamma^{\mu} Q^{\nu}\right)$ \\
\hline 5 & $m^{2} K_{31}=\lambda \omega T_{31}=\lambda \omega\left[Q^{\mu} \gamma^{\nu}+\gamma^{\mu} Q^{\prime \nu}, \mathbb{Z}\right]$ & 2 & $i m K_{25}=-i \lambda T_{25}=\lambda\left(Q^{\mu} \gamma^{\nu}+\gamma^{\mu} Q^{\prime \nu}\right)$ \\
\hline 3 & $m^{2} K_{32}=-\lambda T_{32}=\lambda\left[Q^{\mu} \gamma^{\nu}-\gamma^{\mu} Q^{\prime \nu}, \not{Z}\right]$ & 4 & $i m K_{26}=i \lambda \omega T_{26}=\lambda \omega\left(Q^{\mu} \gamma^{\nu}-\gamma^{\mu} Q^{\prime \nu}\right)$ \\
\hline 1 & $K_{33}=\lambda T_{33}=\lambda\left[\gamma^{\mu}, \gamma^{\nu}\right]$ & 1 & $i m K_{34}=i T_{34}=\left\{\left[\gamma^{\mu}, \gamma^{\nu}\right], \not{\Sigma}\right\}$ \\
\hline
\end{tabular}

with $\alpha=\lambda / \sqrt{1+t}$ and $\beta=\eta_{-} / \alpha$. In any case, this does not affect the CFFs because they are frame-independent.

\section{Formulas}

Dropping the index "E," we collect some useful Euclidean formulas. The $\gamma_{5}$ matrix is defined by

$$
\gamma^{5}=-\gamma^{1} \gamma^{2} \gamma^{3} \gamma^{4}=-\frac{1}{24} \varepsilon^{\mu \nu \rho \sigma} \gamma^{\mu} \gamma^{\nu} \gamma^{\rho} \gamma^{\sigma}
$$

with $\varepsilon^{1234}=1$. It is convenient to define the fully antisymmetric combinations of Dirac matrices via the commutators

$$
\begin{gathered}
{[A, B]=A B-B A,} \\
{[A, B, C]=[A, B] C+[B, C] A+[C, A] B,} \\
{[A, B, C, D]=[A, B, C] D-[B, C, D] A} \\
+[C, D, A] B-[D, A, B] C .
\end{gathered}
$$

Inserting $\gamma$-matrices, this yields the antisymmetric combinations

$$
\begin{aligned}
{\left[\gamma^{\mu}, \gamma^{\nu}\right] } & =\gamma_{5} \varepsilon^{\mu \nu \alpha \beta} \gamma^{\alpha} \gamma^{\beta} \\
\frac{1}{6}\left[\gamma^{\mu}, \gamma^{\nu}, \gamma^{\rho}\right] & =\frac{1}{2}\left(\gamma^{\mu} \gamma^{\nu} \gamma^{\rho}-\gamma^{\rho} \gamma^{\nu} \gamma^{\mu}\right) \\
& =\frac{1}{4}\left\{\left[\gamma^{\mu}, \gamma^{\nu}\right], \gamma^{\rho}\right\}
\end{aligned}
$$

$$
\begin{aligned}
& =-\gamma_{5} \varepsilon^{\mu \nu \rho \sigma} \gamma^{\sigma}, \\
\frac{1}{24}\left[\gamma^{\mu}, \gamma^{\nu}, \gamma^{\alpha}, \gamma^{\beta}\right] & =-\gamma_{5} \varepsilon^{\mu \nu \alpha \beta} .
\end{aligned}
$$

The various contractions of $\varepsilon$-tensors are given by

$$
\begin{aligned}
\varepsilon^{\mu \nu \rho \lambda} \varepsilon^{\alpha \beta \gamma \lambda}= & \delta^{\mu \alpha}\left(\delta^{\nu \beta} \delta^{\rho \gamma}-\delta^{\nu \gamma} \delta^{\rho \beta}\right)+\delta^{\mu \beta}\left(\delta^{\nu \gamma} \delta^{\rho \alpha}-\delta^{\nu \alpha} \delta^{\rho \gamma}\right) \\
& +\delta^{\mu \gamma}\left(\delta^{\rho \beta} \delta^{\nu \alpha}-\delta^{\rho \alpha} \delta^{\nu \beta}\right) \\
\frac{1}{2} \varepsilon^{\mu \nu \lambda \sigma} \varepsilon^{\alpha \beta \lambda \sigma}= & \delta^{\mu \alpha} \delta^{\nu \beta}-\delta^{\mu \beta} \delta^{\nu \alpha} \\
\frac{1}{6} \varepsilon^{\mu \lambda \sigma \tau} \varepsilon^{\alpha \lambda \sigma \tau}= & \delta^{\mu \alpha} \\
\frac{1}{24} \varepsilon^{\lambda \sigma \tau \omega} \varepsilon^{\lambda \sigma \tau \omega}= & 1
\end{aligned}
$$

and the $\varepsilon$-tensor satisfies

$$
a^{\{\mu} \varepsilon^{\alpha \beta \gamma \delta\}}=0
$$

where $a^{\mu}$ is an arbitrary four-vector and $\{\ldots\}$ denotes a symmetrization of indices.

Four-momenta are conveniently expressed through hyperspherical coordinates: 


$$
p^{\mu}=\sqrt{p^{2}}\left[\begin{array}{l}
\sqrt{1-z^{2}} \sqrt{1-y^{2}} \sin \phi \\
\sqrt{1-z^{2}} \sqrt{1-y^{2}} \cos \phi \\
\sqrt{1-z^{2}} y \\
z
\end{array}\right],
$$

and a four-momentum integration reads:

$$
\begin{aligned}
\int \frac{d^{4} p}{(2 \pi)^{4}}= & \frac{1}{(2 \pi)^{4}} \frac{1}{2} \int_{0}^{\infty} d p^{2} p^{2} \int_{-1}^{1} d z \sqrt{1-z^{2}} \\
& \times \int_{-1}^{1} d y \int_{0}^{2 \pi} d \phi .
\end{aligned}
$$

The positive- and negative-energy on-shell spinors for spin- $1 / 2$ particles satisfy the Dirac equations

$$
\begin{aligned}
& (i \not p+m) u(\boldsymbol{p})=0=\bar{u}(\boldsymbol{p})(i \not p+m), \\
& (i \not p-m) v(\boldsymbol{p})=0=\bar{v}(\boldsymbol{p})(i \not \not-m),
\end{aligned}
$$

where the conjugate spinor is $\bar{u}(\boldsymbol{p})=u(\boldsymbol{p})^{\dagger} \gamma^{4}$. Since the on-shell spinors only depend on $\boldsymbol{p}$ they are the same as in Minkowski space; e.g., in the standard representation:

$$
u_{s}(\boldsymbol{p})=\sqrt{\frac{E_{p}+m}{2 m}}\left(\begin{array}{c}
\xi_{s} \\
\frac{\boldsymbol{p} \cdot \boldsymbol{\tau}}{E_{p}+m} \xi_{s}
\end{array}\right)
$$

with

$$
\xi_{+}=\left(\begin{array}{l}
1 \\
0
\end{array}\right), \quad \xi_{-}=\left(\begin{array}{l}
0 \\
1
\end{array}\right), \quad E_{p}=\sqrt{\boldsymbol{p}^{2}+m^{2}} .
$$

We have normalized them to unity,

$$
\begin{aligned}
& \bar{u}_{s}(\boldsymbol{p}) u_{s^{\prime}}(\boldsymbol{p})=-\bar{v}_{s}(\boldsymbol{p}) v_{s^{\prime}}(\boldsymbol{p})=\delta_{s s^{\prime}}, \\
& \bar{u}_{s}(\boldsymbol{p}) v_{s^{\prime}}(\boldsymbol{p})=\bar{v}_{s}(\boldsymbol{p}) u_{s^{\prime}}(\boldsymbol{p})=0,
\end{aligned}
$$

and their completeness relations define the positive- and negative-energy projectors:

$$
\begin{aligned}
& \sum_{s} u_{s}(\boldsymbol{p}) \bar{u}_{s}(\boldsymbol{p})=\frac{-i \not p+m}{2 m}=\Lambda_{+}(p), \\
& \sum_{s} v_{s}(\boldsymbol{p}) \bar{v}_{s}(\boldsymbol{p})=\frac{-i \not p-m}{2 m}=-\Lambda_{-}(p) .
\end{aligned}
$$

Therefore, $\Lambda_{+}(p) u(\boldsymbol{p})=u(\boldsymbol{p})$ and $\Lambda_{-}(p) u(\boldsymbol{p})=0$.

\section{APPENDIX B: TENSOR BASIS}

In the following we derive the tensor basis of the nucleon Compton amplitude given in Table I and Eq. (2.26). To begin with, we follow the construction by Tarrach [29] and define the 34 auxiliary tensors $K_{i}^{\mu \nu}$ in Table XV. The $T_{i}^{\mu \nu}$ are the Euclidean versions of Tarrach's Eq. (2.8) according to the replacement rules in Appendix A. We construct the $K_{i}^{\mu \nu}$ by attaching prefactors of $\lambda, \omega$ or $\lambda \omega$, which makes them even under Bose symmetry and charge conjugation, cf. Eq. (2.19), and powers of the nucleon mass $m$ to make them dimensionless. Thus, the initial nucleon Compton scattering amplitude has the form

$$
\Gamma^{\mu \nu}\left(p, Q^{\prime}, Q\right)=\Lambda_{+}^{f}\left[\sum_{i=1}^{34} g_{i} K_{i}^{\mu \nu}\right] \Lambda_{+}^{i} .
$$

Here we abbreviated the positive-energy projectors from Eq. (2.2) by $\Lambda_{+}^{f, i}$ and the dressing functions $g_{i}$ depend on the variables $\eta_{+}, \eta_{-}, \omega$ and $\lambda$ defined in Eq. (2.6).

That the $K_{i}$ are fully symmetric will be important in what follows, because it implies that the $g_{i}$ are even in both $\omega$ and $\lambda$. The analysis in terms of the $T_{i}$ would complicate the discussion of kinematic singularities; take e.g., the contribution from $K_{33}$ :

$$
g_{33} K_{33}=\lambda g_{33} T_{33}=g_{33}^{\prime} T_{33} .
$$

Clearly, $g_{33}=g_{33}^{\prime} / \lambda$ does not have a kinematic singularity at $\lambda=0$ because $g_{33}^{\prime}$ is odd under photon crossing and therefore must be proportional to $\lambda$.

There are two non-trivial linear dependencies between the $K_{i}^{\mu \nu}$, namely [29]

$$
\begin{gathered}
K_{17}-K_{19}+K_{22}-K_{23}+K_{25}+K_{28}-K_{33} \\
+\left(1+\frac{\eta_{+}-\eta_{-}}{2}\right) \frac{K_{34}}{2}=0
\end{gathered}
$$

and

$$
\begin{aligned}
& \frac{K_{8}+K_{10}}{2}+K_{12}-K_{13}-\frac{K_{24}+K_{26}}{2}+\frac{K_{29}-K_{31}}{4} \\
& -\lambda^{2}\left(K_{2}-K_{3}+K_{28}+\frac{K_{34}}{2}\right) \\
& +\frac{\eta_{+}+\eta_{-}}{2}\left(K_{7}-K_{9}-K_{23}+K_{25}\right) \\
& +\frac{\eta_{+}-\eta_{-}}{4}\left(K_{30}+K_{32}\right) \\
& +\left(\lambda^{2}+\frac{\eta_{+}^{2}-\eta_{-}^{2}-\omega^{2}}{4}\right) K_{33}=0 .
\end{aligned}
$$

These relations hold inside the positive-energy projectors of Eq. (B1). Therefore, only 32 tensors are linearly independent. This is analogous to the discussion of the light-by-light scattering amplitude in Ref. [50]: an $n$-point function depends on $n-1$ momenta, but with increasing $n$ one can only construct a limited number of orthogonal momenta due to the fixed dimension of spacetime. In practice this leads to relations between the basis elements and thus to a smaller number of independent tensors than what can be naively written down.

One must therefore eliminate two tensors in such a way that the resulting 32 coefficients $g_{i}$ do not pick up kinematic singularities. To do so, it is sufficient to eliminate one tensor from the first row in Eq. (B3) and another from the first two rows in Eq. (B4). Within this constraint, any choice is 
equivalent and we choose to eliminate $K_{22}$ from the first equation and $K_{31}$ from the second one. After crossing off these two tensors from Eq. (B1) the sum goes over 32 linearly independent tensors.

\section{Transverse part}

To derive the transverse part $\Gamma_{\perp \perp}^{\mu \nu}$ in Eq. (2.20), we work out the transversality conditions

$$
\begin{gathered}
Q^{\prime \mu} \Gamma^{\mu \nu}\left(p, Q^{\prime}, Q\right) \stackrel{!}{=} 0, \\
Q^{\nu} \Gamma^{\mu \nu}\left(p, Q^{\prime}, Q\right) \stackrel{!}{=} 0 .
\end{gathered}
$$

Either one of these is sufficient as long as we respect photon-crossing and charge-conjugation invariance. For example, the contraction of (B1) with $Q^{\prime \mu}$,

$$
\sum_{\substack{i=1 \\\{22,31\}}}^{34} g_{i} Q^{\prime \mu} K_{i}^{\mu \nu}=\sum_{j=1}^{8} A_{j} K_{j}^{\nu} \stackrel{!}{=} 0,
$$

produces eight linearly independent tensors $K_{j}^{\nu}$ :

$$
\begin{aligned}
& p^{\nu}, \quad Q^{\prime \nu}, \quad Q^{\nu}, \quad p^{\nu} \Psi, \quad Q^{\prime \nu} \Psi, \\
& Q^{\nu} \Varangle, \quad \gamma^{\nu}, \quad\left[\gamma^{\nu}, \Psi\right] \text {. }
\end{aligned}
$$

This leads to eight conditions $A_{j}=0$ for their Lorentzinvariant coefficients and thus eight relations between the dressing functions $g_{i}$. Whereas the $A_{j}$ are either even or odd in $\lambda$, they are superpositions of even and odd pieces in $\omega$ :

$$
A_{j}=A_{j}^{(1)}+\omega A_{j}^{(2)} \stackrel{!}{=} 0 .
$$

Thus we arrive at 16 conditions $A_{j}^{(1)}=0$ and $A_{j}^{(2)}=0$, where it turns out that only 14 are independent.

The resulting relations are rather complicated but they can be solved without divisions: similarly to (B3)-(B4) one can eliminate 14 dressing functions $g_{j}$ (e.g., those for $j=1,2,4,6,9,11,12,14,19,21,23,27,28,33)$ without dividing by terms depending on the kinematic variables $\eta_{+}, \eta_{-}, \omega^{2}$ and $\lambda^{2}$. If we relabel the independent functions by $c_{i}$ with $i=1 \ldots 18$, the relations take the form $g_{j}=g_{j}\left(c_{1}, \ldots c_{18}\right)$ and reinserting them into (B1) yields the transverse Compton amplitude

$$
\Gamma_{\perp \perp}^{\mu \nu}=\Lambda_{+}^{f}\left[\sum_{i=1}^{18} c_{i} X_{i}^{\mu \nu}\right] \Lambda_{+}^{i} .
$$

The transverse tensors $X_{i}$ are given in Table XVI and identical to those in Table I in the main text.

We did check other choices of eliminating two tensors from (B3)-(B4) within the aforementioned constraints. They all produced equivalent bases in the sense that the determinants of the transformation matrices between the
TABLE XVI. Basis of Table I expressed through the elementary tensors $K_{i}$ of Table XV. The equalities hold inside the nucleon spinors since we exploited the relations (B3)-(B4). For $X_{15}$ and $X_{16}$ we abbreviated $\tilde{K}=X_{6}+4 K_{6}+2 K_{21}+K_{33}$.

$X_{1}=\lambda^{2} K_{1}+\eta_{-} K_{6}+K_{7}$

$X_{2}=\eta_{-} K_{1}-K_{3}$

$X_{3}=\left(\eta_{+}^{2}-\omega^{2}\right) K_{1}+\eta_{-} K_{2}-\eta_{+} K_{4}+K_{5}$

$X_{4}=\lambda^{2} K_{2}+\left(\eta_{+}^{2}-\omega^{2}\right) K_{6}+\eta_{+} K_{9}-K_{10}$

$X_{5}=\lambda^{2}\left(-2 \eta_{+} K_{1}+K_{4}\right)-\eta_{+} K_{7}-K_{8}+\eta_{-} K_{9}$

$X_{6}=-K_{28}+K_{33}-\frac{1}{2} K_{34}$

$X_{7}=2\left(-K_{19}+K_{22}-K_{23}+\frac{1}{4} \eta_{+} K_{34}\right)$

$X_{8}=2\left(K_{20}-\eta_{+} K_{22}-K_{24}-\frac{1}{4} \omega^{2} K_{34}\right)$

$X_{9}=4\left(K_{18}-\eta_{-} K_{22}-K_{26}\right)+X_{8}$

$X_{10}=-2 K_{11}+\eta_{-} K_{21}+2 K_{25}-\frac{1}{4} \eta_{-} K_{34}+2 X_{1}$

$X_{11}=4 K_{16}+2 \lambda^{2}\left(K_{21}+\frac{1}{4} K_{34}\right)+\lambda^{2} X_{6}+\frac{1}{4} X_{13}$

$X_{12}=-4 \lambda^{2} K_{1}-2 K_{7}+4 K_{11}-2 K_{25}+K_{32}+\eta_{-} K_{33}$

$X_{13}=-4 \lambda^{2} K_{2}+2 K_{10}+4 K_{12}-2 K_{24}+K_{29}$

$-2 \eta_{+}\left(K_{9}+K_{23}-\frac{1}{2} K_{30}\right)+\left(\eta_{+}^{2}-\omega^{2}\right) K_{33}$

$X_{14}=2\left(-K_{8}+K_{26}\right)+\eta_{-}\left(2 K_{22}+K_{27}\right)+K_{31}$

$X_{15}=2\left(-K_{9}+K_{19}\right)+K_{27}-K_{30}-\eta_{+} \tilde{K}-X_{7}$

$X_{16}=2\left(-K_{10}+K_{20}\right)+\eta_{+} K_{27}+K_{29}-\omega^{2} \tilde{K}+X_{8}$

$X_{17}=-K_{14}+\eta_{+}\left(2 K_{11}-K_{25}\right)+\eta_{-} K_{23}+K_{26}$

$\underline{\underline{X_{18}}=K_{15}-\omega^{2}\left(2 K_{11}-K_{25}\right)+\eta_{-} K_{24}-\eta_{+} K_{26}}$

bases are constant and not momentum-dependent (so they can never vanish or diverge).

The procedure by Bardeen and Tung [30] and Tarrach [29] for deriving the transverse basis is slightly different from ours. In that case one enforces transversality by acting with projectors on the initial amplitude:

$$
\frac{t_{Q^{\prime} Q}^{\mu \alpha}}{Q \cdot Q^{\prime}} \Gamma^{\alpha \beta} \frac{t_{Q^{\prime} Q}^{\beta \nu}}{Q \cdot Q^{\prime}} \stackrel{!}{=} \Gamma^{\mu \nu},
$$

which gives 18 tensors with single and double poles in the variable $Q \cdot Q^{\prime}$. By forming appropriate linear combinations one then eliminates as many poles as possible, multiplies the remaining double-pole structures with $Q \cdot Q^{\prime}$, repeats, and finally multiplies the single-pole tensors by $Q \cdot Q^{\prime}$. In contrast to Eqs. (B5)-(B7), however, this does not automatically lead to a minimal basis. Tarrach derives the tensors $\tau_{1 \ldots 18}$, given in Table XVII, but notes that the resulting basis is not minimal due to $\tau_{5}, \tau_{15}$ and $\tau_{16}$, which are subsequently exchanged with new tensors $\tau_{19}, \tau_{20}$ and $\tau_{21}$ to form a minimal basis. Written in terms of the $X_{i}$, the problem with these tensors is evident as one can see in the table: e.g., all coefficients of the $X_{i}$ in the equation for $\tau_{5}$ are momentumdependent and thus the determinant of the basis transformation from $\tau_{1 \ldots 18}$ to $X_{1 \ldots 18}$ would depend on the kinematics. Phrased differently, the crossing- and chargeconjugation symmetric combination $\lambda \omega \tau_{5}$ has a higher photon momentum power (namely $n=6$ ) than its replacement $\tau_{19} \sim X_{4}$ with $n=4$; cf. the discussion in Sec. II B. 
TABLE XVII. Tarrach's basis for the Compton amplitude [29]. The $\tau_{i}$ are the transverse tensors in Eqs. (12-13) therein; with appropriate prefactors $\lambda, \omega$ or $\lambda \omega$ they become symmetric under photon crossing and charge conjugation. The $X_{i}$ are our Compton tensors from Table XVI.

\begin{tabular}{ll}
\hline \hline$\tau_{1} / m^{2}=X_{2}$ & $\lambda \tau_{12} / m^{4}=X_{5}-\frac{1}{2} \eta_{+} X_{12}+X_{17}$ \\
$\tau_{2} / m^{4}=-X_{3}$ & $\lambda \omega \tau_{13} / m^{4}=\left(\eta_{+}^{2}-\omega^{2}\right) X_{1}+\lambda^{2} X_{3}-\eta_{-} X_{4}+\eta_{+} X_{5}-\frac{1}{2} \omega^{2} X_{12}-X_{18}$ \\
$\tau_{3} / m^{4}=-X_{1}$ & $\omega \tau_{14} / m^{4}=X_{14}$ \\
$\lambda \tau_{4} / m^{4}=-X_{5}$ & $\omega \tau_{20} / m^{4}=-\left(\omega^{2} X_{6}-2 X_{8}+X_{16}\right)$ \\
$\tau_{19} / m^{6}=2 X_{4}$ & $\tau_{21} / m^{4}=-\left(\eta_{+} X_{6}+2 X_{7}+X_{15}\right)$ \\
$\lambda \tau_{6} / m^{4}=-\frac{1}{4} X_{13}$ & $\lambda \tau_{17} / m^{2}=-X_{12}$ \\
$\lambda \tau_{7} / m^{3}=-2 \lambda^{2} X_{6}+2 X_{11}-\frac{1}{2} X_{13}$ & $\tau_{18} / m^{3}=4 X_{6}-2 X_{7}$ \\
$\tau_{8} / m^{3}=-\frac{1}{2} X_{7}$ & \\
$\omega \tau_{9} / m^{3}=-\frac{1}{2} X_{8}$ & $\lambda \omega \tau_{5} / m^{4}=\left(\eta_{+}^{2}-\omega^{2}\right) X_{1}+\lambda^{2} X_{3}-\eta_{-} X_{4}+\eta_{+} X_{5}$ \\
$\tau_{10} / m^{4}=4 X_{10}+2 X_{12}$ & $\lambda \omega \tau_{15} / m^{4}=\omega^{2}\left(\eta_{-} X_{6}+2 X_{10}+X_{12}\right)-\eta_{-}\left(2 X_{8}-X_{16}\right)-\eta_{+} X_{14}$ \\
$\omega \tau_{11} / m^{3}=\frac{1}{4}\left(X_{8}-X_{9}\right)$ & $\lambda \tau_{16} / m^{4}=\eta_{+}\left(\eta_{-} X_{6}+2 X_{10}+X_{12}\right)+\eta_{-}\left(2 X_{7}+X_{15}\right)-X_{14}$ \\
\hline \hline
\end{tabular}

For these reasons we prefer the more direct method of Eqs. (B5)-(B7) for deriving the basis, because it is failsafe and also provides a safety check: if it were not possible to solve (B7) without divisions, this would point to a problem with minimality. Fortunately, in the case of Compton scattering there is no such problem. Our main reasons for working with the $X_{i}$ instead of Tarrach's (modified) basis are a cleaner physical interpretation (see the remarks at the end of Sec. B), a simpler form of Table I, and simpler expressions for Tables IV, VI and IX.

As discussed in connection with Eq. (2.44), it is still possible to identify kinematic limits where kinematic singularities cannot be avoided. In that case the 18 tensors $X_{i}$ collapse into a set of fewer tensors whose coefficients are linear combinations of the $c_{i}$ but with singular denominators. However, this does not change the fact that the $c_{i}$ themselves are still finite in those limits. Take e.g., our results in Fig. 16: the $\hat{c}_{i}$ obtained from the intermediate nucleon resonances are nonsingular everywhere inside the TPE cone, although the cone contains the VVCS limit $\omega=0$. This means we can go arbitrarily close to that limit and extract the $\hat{c}_{i}$, which also remain finite in the limit. On the other hand, had we calculated directly in the VVCS limit, the $\hat{c}_{i}$ would have collapsed into fewer functions with kinematic singularities.

In any case, this situation does not affect the kinematic limits of RCS, VCS and the forward limit where direct measurements are possible. This is evident from the discussion below Eq. (2.30), Eqs. (2.36)-(2.37), and Eq. (2.41), as well as the following subsection B 2: in all those cases the $X_{i}$ collapse into fewer tensors but the respective coefficients do not pick up kinematic singularities. Hence, in principle the CFFs (or their combinations) can be measured directly in these limits.

\section{Kinematic limits}

In the following we collect the relations between the $K_{i}$ in Table XV in the various kinematic limits, which leads to the reduced transverse bases discussed in Sec. II C. We further relate the CFFs in those limits to some common amplitude choices employed in the literature.

RCS: Here the condition $\omega=0$ eliminates the tensors

$$
\begin{array}{cccc}
K_{5} & K_{15} & K_{22} & K_{27} \\
K_{8} & K_{18} & K_{24} & K_{29} \\
K_{10} & K_{20} & K_{26} & K_{31}
\end{array}
$$

from the basis. In addition, applying polarization vectors for the on-shell photon momenta has the same effect as crossing off tensors which contain instances of either $Q^{\prime \mu}$ or $Q^{\nu}$, so that also

$$
\begin{array}{lll}
K_{2} & K_{12} & K_{23} \\
K_{4} & K_{14} & K_{30} \\
K_{9} & K_{19} &
\end{array}
$$

vanish in RCS. From Table XVI one then infers that only the transverse tensors $X_{1}, X_{2}, X_{6}, X_{10}, X_{11}$ and $X_{12}$ survive in RCS. The relations between our CFFs and the RCS amplitudes $A_{i}\left(\eta_{-}, \lambda\right)$ defined by L'vov et al. [51,52] are given in Table XVIII.

VCS: The same strategy applied to VCS amounts to dropping instances of $Q^{\prime \mu}$ only. With $\eta_{+}=\omega$ this implies

TABLE XVIII. Relations between the RCS amplitudes $A_{i}$ of Refs. [51,52] and our CFFs in RCS $\left(\eta_{+}=\omega=0\right)$.

$$
\left[\begin{array}{c}
A_{1} \\
A_{2} \\
A_{3} \\
A_{4} \\
A_{5} \\
A_{6}
\end{array}\right]=-\frac{e^{2}}{m^{3}}\left[\begin{array}{c}
\frac{1}{4}\left(\eta_{-}-2\right) c_{1}+c_{2}+\frac{1}{2} \eta_{-} c_{10}+\lambda^{2} c_{11} \\
-\left(c_{6}+c_{10}\right) \\
-\left(\frac{1}{2} c_{1}+c_{10}-c_{11}\right) \\
c_{11} \\
\frac{1}{2}\left(\eta_{-}-2\right) c_{11}+2 c_{12} \\
c_{10}+\frac{1}{2}\left(\eta_{-}-2\right) c_{11}
\end{array}\right]
$$




$$
\begin{array}{ll}
K_{2}=0, & K_{12}=0, \\
K_{5}=\eta_{+} K_{4}, & K_{15}=\eta_{+} K_{14}, \\
K_{10}=\eta_{+} K_{9}, & K_{24}=-\eta_{+} K_{23}, \\
K_{20}=\eta_{+} K_{19}, & K_{29}=-\eta_{+} K_{30}
\end{array}
$$

which induce the linear relations (2.36) between the $X_{i}$. The resulting CFFs are those in Eq. (2.37).

The relations between the Compton tensors $T_{i}$ employed by Drechsel et al., defined in Appendix A of Ref. [31], with Tarrach's $\tau_{i}$ are given by:

$$
\begin{array}{ll}
T_{1}=-\tau_{1}, & T_{6 \ldots 18}=\tau_{6 \ldots 18}, \\
T_{2}=-4 \tau_{3}, & \\
T_{3}=\tau_{2}, & T_{19}=2 \tau_{19}, \\
T_{4}=2 \tau_{4}, & T_{20}=\tau_{20}, \\
T_{5}=-2 \tau_{5}, & T_{21}=\tau_{21} .
\end{array}
$$

These are subsequently used to define the VCS tensors $\rho_{i}$ and corresponding dressing functions $f_{i}$, cf. Eq. (A10) in [31]. Their relations with our CFFs are given in Table XIX. The nucleon's generalized polarizabilities can then be reconstructed using Eqs. (3)-(8) in Ref. [7] or in a manifestly covariant form via Eq. (A1) in Ref. [9]. Note that in the conventions of Drechsel et al. the average nucleon and photon momenta differ by a factor 2 and one has to interchange the Lorentz indices $\mu \leftrightarrow \nu$.

FWD: In the doubly-virtual forward limit the condition $\omega=0$ disposes again of the tensors in Eq. (B10). In addition one has $Q^{\prime \mu}=Q^{\mu}$, but without any polarization vectors because the photons are still virtual. Because the incoming and outgoing nucleon momenta are the same, one exploits the identities

TABLE XIX. Relations between the $f_{i}$ defined in Ref. [31] and our CFFs in VCS $\left(\eta_{+}=\omega\right)$. The relations between the nucleon's generalized polarizabilities and the $f_{i}$ can be found in Refs. [7,9]; the $f_{i}$ in [7,31] are identical to the $A_{i}$ in Ref. [9]. We abbreviated $\bar{c}_{7}=c_{7}-\eta_{+} c_{8}, \bar{c}_{15}=c_{15}+\eta_{+} c_{16}$ and $\bar{c}_{17}=c_{17}-\eta_{+} c_{18}$.

$$
\begin{aligned}
& m^{3} f_{1}=-c_{2} \\
& m^{5} f_{2}=\frac{1}{4} c_{1} \\
& m^{5} f_{3}=-\frac{1}{2} \lambda\left(c_{5}-\bar{c}_{17}\right) \\
& m^{4} f_{4}=\frac{1}{2} \lambda c_{11} \\
& m^{4} f_{5}=-\left(c_{6}+2 \bar{c}_{7}-2 \eta_{+} c_{9}+\lambda^{2} c_{11}-\left(\eta_{+}+4\right) \bar{c}_{15}\right) \\
& m^{5} f_{6}=\frac{1}{4} c_{10} \\
& m^{4} f_{7}=-4 \eta_{+} c_{9} \\
& m^{5} f_{8}=\lambda \bar{c}_{17} \\
& m^{5} f_{9}=\eta_{+} c_{14} \\
& m^{3} f_{10}=\frac{1}{2} \lambda\left(c_{10}-2 c_{12}-\eta_{+} \bar{c}_{17}\right) \\
& m^{4} f_{11}=\frac{1}{4}\left(c_{6}+\lambda^{2} c_{11}-\eta_{+} \bar{c}_{15}\right) \\
& m^{5} f_{12}=-\bar{c}_{15} \\
& \hline \hline
\end{aligned}
$$

$$
\Lambda_{+}(p) \gamma^{\mu} \Lambda_{+}(p)=\frac{p^{\mu}}{i m} \Lambda_{+}(p)
$$

and

$$
\begin{aligned}
& \Lambda_{+}(p)\left[p^{\mu} \gamma^{\nu}-\gamma^{\mu} p^{\nu}, \gamma^{\rho}\right] \Lambda_{+}(p) \\
& \quad=\Lambda_{+}(p)\left(\frac{i m}{3}\left[\gamma^{\mu}, \gamma^{\nu}, \gamma^{\rho}\right]-\left[\gamma^{\mu}, \gamma^{\nu}\right] p^{\rho}\right) \Lambda_{+}(p)
\end{aligned}
$$

to obtain further relations between the $K_{i}$ :

$$
\begin{aligned}
K_{3} & =\frac{1}{2} K_{4}=K_{2}, \\
K_{9} & =K_{17}=K_{19}=-K_{23}=-K_{25}=K_{7}, \\
K_{11} & =\lambda^{2} K_{1}, \quad K_{12}=K_{13}=\frac{1}{2} K_{14}=\lambda^{2} K_{2}, \\
K_{16} & =\lambda^{2} K_{6}, \quad K_{21}=-2 K_{6}, \\
K_{28} & =K_{33}-\frac{1}{2} K_{34}, \quad K_{32}=K_{30}
\end{aligned}
$$

In total only seven tensors $K_{1}, K_{2}, K_{6}, K_{7}, K_{30}, K_{33}$ and $K_{34}$ remain independent. The resulting four transverse tensors in the forward limit are given in Eq. (2.39) and the corresponding CFFs in Eq. (2.41).

\section{Nontransverse part}

Next, we work out the remaining nontransverse tensors of the basis, i.e., the $\Gamma_{\perp}^{\mu \nu}$ and $\Gamma_{\mathrm{G}}^{\mu \nu}$ from Eq. (2.20). For the physical amplitude they are irrelevant because their coefficients vanish due to gauge invariance, but projecting onto the full 32-dimensional basis including all terms serves as a useful test of gauge invariance.

The "partially transverse" piece $\Gamma_{\perp}^{\mu \nu}$ is subject to the weaker constraint

$$
Q^{\prime \mu} \Gamma^{\mu \nu}\left(p, Q^{\prime}, Q\right) Q^{\nu} \stackrel{!}{=} 0 .
$$

To derive it, we lift the requirement of gauge invariance and add the 14 tensors belonging to the $g_{j}$ [given above Eq. (B8)] that we previously eliminated:

$$
\Gamma^{\mu \nu}=\Lambda_{+}^{f}\left[\sum_{j} g_{j} K_{j}^{\mu \nu}+\sum_{i=1}^{18} c_{i} X_{i}^{\mu \nu}\right] \Lambda_{+}^{i} .
$$

Since the transverse tensors $X_{i}$ already satisfy Eq. (B14) the condition only affects the first sum. Its contraction with $Q^{\prime \mu}$ and $Q^{\nu}$ generates two independent structures proportional to $\mathbb{1}$ and $\not{Z}$,

$$
\sum_{j} g_{j} Q^{\prime \mu} K_{j}^{\mu \nu} Q^{\nu}=A_{1}^{\prime}+A_{2}^{\prime} i \lambda \stackrel{!}{=}^{\prime} 0,
$$

with two resulting conditions $A_{1}^{\prime}=A_{2}^{\prime}=0$. This leaves 12 independent functions; if we relabel them by $c_{i}$ with $i=19 \ldots 30$, the result is 
TABLE XX. Nontransverse basis tensors for the Compton amplitude. Taken together with those in Table XVI, they form a complete basis.

\begin{tabular}{ll}
\hline \hline$X_{19}=\eta_{-} K_{2}-\left(\eta_{+}^{2}-\omega^{2}\right) K_{1}$ & $X_{27}=K_{22}$ \\
$X_{20}=\eta_{-} K_{6}-\lambda^{2} K_{1}$ & $X_{28}=K_{26}$ \\
$X_{21}=2 \eta_{+} K_{1}-K_{4}$ & $X_{29}=K_{27}$ \\
$X_{22}=2 \eta_{+} K_{6}-K_{9}$ & $X_{30}=K_{34}$ \\
$X_{23}=2 K_{11}-K_{25}$ & \\
$X_{24}=2 K_{12}+\left(\eta_{+}^{2}-\omega^{2}\right) K_{21}$ & $X_{31}=K_{1}$ \\
$X_{25}=K_{19}-K_{23}-2 \eta_{+} K_{21}$ & $X_{32}=K_{21}$ \\
$X_{26}=K_{28}+K_{33}+4 K_{21}+8 K_{6}$ & \\
\hline \hline
\end{tabular}

$$
\Gamma_{\perp}^{\mu \nu}=\Lambda_{+}^{f}\left[\sum_{i=19}^{30} c_{i} X_{i}^{\mu \nu}\right] \Lambda_{+}^{i}
$$

where the tensors $X_{19 . .30}$ are collected in Table XX.

Unfortunately, here it is no longer possible to solve the system (B15) without divisions, which means that some of the resulting tensors acquire kinematic singularities. Those are the ones proportional to $X_{19}$ and $X_{20}$, whose original form as a result of the equations is $X_{19} / \eta_{-}$and $X_{20} / \eta_{-}$. Therefore, they must be multiplied by $\eta_{-}$which removes the kinematic singularities at $\eta_{-}=0$ and in the process raises their photon momentum powers.

That the $X_{19 \ldots 30}$ satisfy Eq. (B14) is a simple check; for example, Table XV entails

$$
Q^{\prime \mu} K_{22}^{\mu \nu} Q^{\nu}=\frac{\omega}{i m}\left(p \cdot Q^{\prime} Q-Q^{\prime} p \cdot Q\right)=i m \lambda \omega \not \Delta,
$$

which vanishes in the contraction with the positive-energy projectors. In analogy to Eq. (2.26), it is possible to recast the $X_{19 \ldots 30}$ in a form where the (partial) transversality is manifest. For example, using the definition (2.25):

$$
\begin{aligned}
X_{21}^{\mu \nu} & =\frac{1}{m^{2}}\left(t_{Q^{\prime} Q^{\prime}}^{\mu \nu}+t_{Q Q}^{\mu \nu}\right), \\
X_{23}^{\mu \nu} & =\frac{i \lambda}{m}\left(t_{Q^{\prime} \gamma}^{\mu \nu}+t_{\gamma Q}^{\mu \nu}\right), \\
X_{28}^{\mu \nu} & =\frac{i \lambda \omega}{m}\left(t_{Q^{\prime} \gamma}^{\mu \nu}-t_{\gamma Q}^{\mu \nu}\right) .
\end{aligned}
$$

Finally, the remaining nontransverse part

$$
\Gamma_{\mathrm{G}}^{\mu \nu}=\Lambda_{+}^{f}\left[c_{31} X_{31}^{\mu \nu}+c_{32} X_{32}^{\mu \nu}\right] \Lambda_{+}^{i}
$$

depends on the two tensors $X_{31}=K_{1}$ and $X_{32}=K_{21}$ corresponding to the coefficients that we eliminated in the solution of Eq. (B15). In total, the sum of Eqs. (B8), (B16) and (B18) constitutes a complete 32-dimensional tensor basis according to Eq. (2.20).

\section{Effects of breaking gauge invariance}

Finally we return to the question posed in Sec. IV C: what are the consequences of breaking gauge invariance on purpose? To investigate this, we consider the nucleon Born term from Eq. (4.1) but implement a nucleon-photon vertex that differs from the Dirac form (4.18). For example:

$$
\Gamma^{\mu}(k, Q)=i\left[F_{1} G_{1}^{\mu}+F_{2} \frac{T_{3}^{\mu}}{2}+\alpha T_{4}^{\mu}\right]
$$

with the usual Dirac and Pauli form factors $F_{1,2}\left(Q^{2}\right)$, but including the tensor $T_{4}^{\mu}$ from Table II with a constant coefficient $\alpha$. For simplicity, let us set $F_{1}=1$ and $F_{2}=0$. Then for $\alpha=0$ one obtains the CFFs for a pointlike Dirac particle: $\tilde{c}_{1}=-4$ and $\tilde{c}_{10}=2$.

After switching on $\alpha$, the Compton amplitude is no longer gauge invariant. However, by projecting it onto its full 32-dimensional tensor basis,

$$
\Gamma^{\mu \nu}=\Gamma_{\mathrm{G}}^{\mu \nu}+\Gamma_{\perp}^{\mu \nu}+\Gamma_{\perp \perp}^{\mu \nu}
$$

with the transverse part $\Gamma_{\perp \perp}^{\mu \nu}$ from Eq. (B8) and the remainders $\Gamma_{\perp}^{\mu \nu}$ and $\Gamma_{\mathrm{G}}^{\mu \nu}$ from Eqs. (B16) and (B18), we can isolate the terms that violate gauge invariance and work out the effects on the transverse CFFs.

The result for the simple example above is given in Table XXI. The transverse CFFs pick up extra terms which depend on $\alpha$, but in addition we have also generated a gauge part $\propto X_{30}$. As in Eq. (4.21) we quote the residues $\tilde{c}_{i}$ for the transverse parts but the $\mathrm{CFF} c_{30}$ itself for the gauge part: only the transverse terms contain the nucleon poles whereas they drop out in the gauge part.

Note also that no additional kinematic singularity has been generated in any CFF. Had we simply performed a transverse projection of the full amplitude, both gauge and transverse parts would have collapsed into 18 transverse functions; however, because the gauge part has a lower photon momentum power it will produce kinematic singularities in those functions.

This principle can be taken further to test off-shell effects in the nucleon Born term within a hadronic description

TABLE XXI. Compton form factors for a pointlike nucleon but with an extra term $\propto T_{4}^{\mu}$ that violates gauge invariance, see Eq. (B19).

\begin{tabular}{ll}
\hline \hline$\tilde{c}_{1}=-4+A-B+D$ & $\tilde{c}_{11}=-4 \alpha^{2}$ \\
$\tilde{c}_{2}=A+\eta_{-}(4 \alpha-C)$ & $\tilde{c}_{12}=2 \alpha-C$ \\
$\tilde{c}_{6}=-A-2 \alpha \eta_{-}$ & $\tilde{c}_{15}=-\alpha^{2} \eta_{-}$ \\
$\tilde{c}_{10}=2-4 \alpha-\frac{1}{2}(A-B+D)$ & $c_{30}=-\frac{1}{2} \alpha$ \\
$A=\alpha^{2}\left(\eta_{-}^{2}-4 \lambda^{2}\right)$ & $C=\alpha^{2}\left(2 \eta_{+}-\eta_{-}+4\right)$ \\
$B=\alpha^{2}\left(\eta_{+}^{2}-\omega^{2}\right)$ & $D=4 \alpha\left(\eta_{+}-\alpha \eta_{-}\right)$ \\
\hline \hline
\end{tabular}



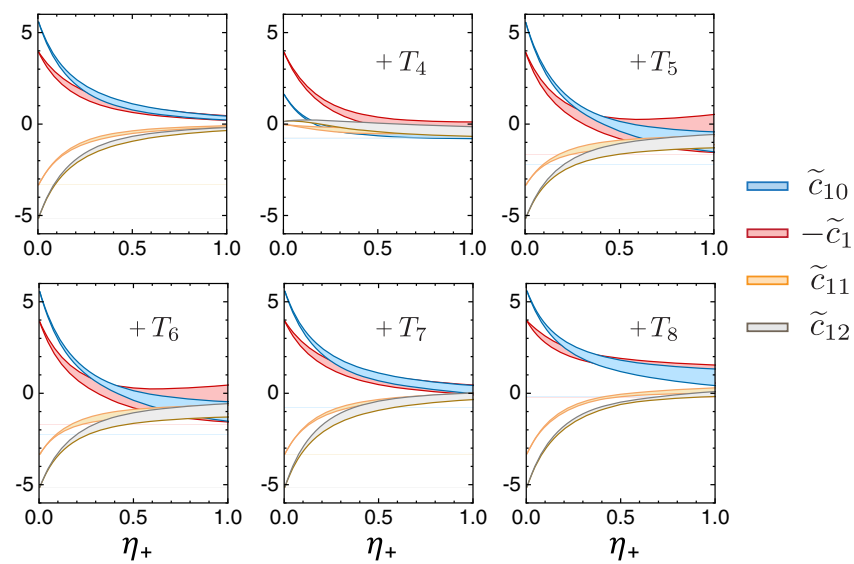

FIG. 20. Compton form factor residues of the nucleon Born term from an off-shell nucleon-photon vertex that violates electromagnetic gauge invariance. The upper left panel shows the original result from Fig. 5 where gauge invariance is preserved. In the remaining panels we switch on $T_{4 \ldots 8}^{\mu}$ as in Eq. (B21), with $\alpha=1$ in all cases.

[144]. To do so, we restore the proton's Dirac and Pauli form factors in Eq. (B19) but add other tensors with a simple momentum dependence of the form

$$
\alpha T_{4}^{\mu} \rightarrow \frac{\alpha}{\left(1+Q^{2} / m^{2}\right)} T_{i}^{\mu},
$$

with $i \neq 1$ and $i \neq 3$. After the projection we drop the unphysical gauge parts and consider the transverse CFFs only. In Fig. 20 we show a few selected results for the leading transverse CFFs obtained with $\alpha=1$. Without the additional tensors (upper left panel) they are identical to those in Fig. 5. As one can see, breaking gauge invariance has rather modest effects on the transverse part of the Compton amplitude because the CFFs do not change their form dramatically. Thus, even if off-shell effects played a role (as far as that can be judged within an effective hadronic theory) their correct implementation leads back to results which resemble the on-shell forms.

This observation is useful also in a different context, namely in microscopic calculations of Compton scattering. In that case the complete expression for the Compton amplitude in terms of quark and gluon d.o.f. has been derived based on electromagnetic gauge invariance $[26,27]$. As usual only the sum of all diagrams is gauge invariant but not the individual terms. Unfortunately, some of those diagrams are numerically hard to calculate. Keeping only parts of the results, even if they provide the dominant contributions, would indeed be useless if one cannot quantify the effects of breaking gauge invariance. For example, with a naive transverse projection the resulting CFFs would be contaminated by unphysical kinematic singularities. The separation (B20) resolves the problem: one can project the dominant diagrams onto the complete basis, which ensures both transversality and the absence of kinematic singularities, and subsequently retain the transverse CFFs. This leads to well-defined expressions, which can be systematically improved upon, where the subleading diagrams would mainly serve to cancel the gauge parts because the sum of all diagrams is known to be gauge invariant.

\section{APPENDIX C: SPIN-3/2 LAGRANGIANS AND POINT TRANSFORMATIONS}

In this Appendix we collect some further properties of spin-3/2 Lagrangians. We drop the label " $R$ " that was used in the main text and denote the mass of the spin-3/2 particle by $m$, its tree-level propagator by $S^{\alpha \beta}$, and the nucleon-toresonance transition vertex by $\Gamma^{\alpha \mu}$.

The free spin-3/2 Lagrangian

$$
\mathcal{L}=\bar{\psi}^{\alpha} \Lambda^{\alpha \beta} \psi^{\beta}, \quad \Lambda^{\alpha \beta}=-\frac{i}{2}\left\{\sigma^{\alpha \beta}, i \not k+m\right\}
$$

with $i \not k=\not \partial$ is a special case of more general possible forms that are related to each other by point transformations $[47,65,68]$. Let us define the transverse and longitudinal projectors onto $\gamma$-matrices,

$$
\mathbb{P}_{\perp}^{\alpha \beta}=\delta^{\alpha \beta}-\frac{1}{4} \gamma^{\alpha} \gamma^{\beta}, \quad \mathbb{P}_{\|}^{\alpha \beta}=\frac{1}{4} \gamma^{\alpha} \gamma^{\beta}
$$

with the properties

$$
\mathbb{P}_{\perp}^{\alpha \beta} \gamma^{\beta}=0, \quad \mathbb{P}_{\|}^{\alpha \beta} \gamma^{\beta}=\gamma^{\alpha}, \quad \mathbb{P}_{\perp}^{\alpha \gamma} \mathbb{P}_{\|}^{\gamma \beta}=0 .
$$

The so-called point transformation tensors

$$
\Theta^{\alpha \beta}(\lambda)=\mathbb{P}_{\perp}^{\alpha \beta}+\lambda \mathbb{P}_{\|}^{\alpha \beta}
$$

form a group:

$$
\begin{aligned}
\Theta^{\alpha \gamma}(\lambda) \Theta^{\gamma \beta}\left(\lambda^{\prime}\right) & =\Theta^{\alpha \beta}\left(\lambda \lambda^{\prime}\right), \\
\Theta^{\alpha \gamma}(\lambda) \Theta^{\gamma \beta}\left(\lambda^{-1}\right) & =\delta^{\alpha \beta},
\end{aligned}
$$

where the group parameter $\lambda$ is the coefficient of the longitudinal part in $\Theta^{\alpha \beta}(\lambda)$. The general form of the inverse propagator $\Lambda^{\alpha \beta}$ can then be written as [47]

$$
\Lambda^{\alpha \beta}(\xi)=\Theta^{\alpha \gamma}\left(\frac{1}{\xi}\right) \Lambda^{\gamma \delta}(\xi=1) \Theta^{\delta \beta}\left(\frac{1}{\xi}\right) .
$$

It depends on a gauge parameter $\xi$, where $\xi=1$ corresponds to the "Rarita-Schwinger gauge" and $\Lambda^{\alpha \beta}(\xi=1)$ is the expression in Eq. (C1). ${ }^{4}$ From Eqs. (C5)-(C6) one has the general relation

$$
\Lambda^{\alpha \beta}(\xi)=\Theta^{\alpha \gamma}\left(\frac{\xi^{\prime}}{\xi}\right) \Lambda^{\gamma \delta}\left(\xi^{\prime}\right) \Theta^{\delta \beta}\left(\frac{\xi^{\prime}}{\xi}\right),
$$

\footnotetext{
${ }^{4}$ To compare with the notation in the literature, e.g., [47,145], use $\xi=-1 /(1+2 A)$ and $\lambda=1+4 a$.
} 
which entails that the Lagrangian $\mathcal{L}=\bar{\psi}^{\alpha} \Lambda^{\alpha \beta}(\xi) \psi^{\beta}$ is invariant under the point transformation

$$
\xi \rightarrow \xi^{\prime}, \quad \psi^{\alpha} \rightarrow \psi^{\prime \alpha}=\Theta^{\alpha \beta}\left(\frac{\xi^{\prime}}{\xi}\right) \psi^{\beta} .
$$

Defining $\Omega=i \not k+m$, one can further show that Eq. (C6) is identical to

$$
\begin{aligned}
\Lambda^{\alpha \beta}(\xi)= & \mathbb{P}_{\perp}^{\alpha \gamma} \Omega \mathbb{P}_{\perp}^{\gamma \beta}-\frac{3}{\xi^{2}} \mathbb{P}_{\|}^{\alpha \gamma} \Omega \mathbb{P}_{\|}^{\gamma \beta} \\
& -\frac{1}{\xi}\left(\mathbb{P}_{\perp}^{\alpha \gamma} \Omega \mathbb{P}_{\|}^{\gamma \beta}+\mathbb{P}_{\|}^{\alpha \gamma} \Omega \mathbb{P}_{\perp}^{\gamma \beta}\right) .
\end{aligned}
$$

The spin-3/2 propagator is the inverse of $\Lambda^{\alpha \beta}(\xi)$ in momentum space and therefore it satisfies

$$
S^{\alpha \beta}(\xi)=\Theta^{\alpha \gamma}(\xi) S^{\gamma \delta}(\xi=1) \Theta^{\delta \beta}(\xi),
$$

where $S^{\alpha \beta}(\xi=1)$ is the Rarita-Schwinger propagator in Eq. (6.3). The explicit form of the general tree-level propagator is

$$
\begin{aligned}
S^{\alpha \beta}(\xi)= & \frac{-i \not k+m}{k^{2}+m^{2}} \Delta^{\alpha \beta}+\frac{(1-\xi)^{2}}{24 i m^{2}} \gamma^{\alpha} \not k \gamma^{\beta} \\
& -\frac{1-\xi}{6 i m^{2}}\left(k^{\alpha} \gamma^{\beta}+\gamma^{\alpha} k^{\beta}\right)+\frac{1-\xi^{2}}{12 m} \gamma^{\alpha} \gamma^{\beta},
\end{aligned}
$$

where only the first term survives for $\xi=1$. Using the spin$3 / 2$ and spin-1/2 projectors defined in Eqs. (6.5)-(6.7) and (6.10), the propagator can also be written as [146]

$$
\begin{aligned}
S^{\alpha \beta}(\xi)= & \frac{-i \not k+m}{k^{2}+m^{2}} \mathbb{P}_{3 / 2}^{\alpha \beta}+\frac{1-\xi}{4 m}\left[(1+\xi)+(1-\xi) \frac{i \not k}{2 m}\right] \mathbb{P}_{11}^{\alpha \beta} \\
& +\frac{3+\xi}{12 m}\left[(3-\xi)-(3+\xi) \frac{i \not k}{2 m}\right] \mathbb{P}_{22}^{\alpha \beta} \\
& +\frac{3+\xi^{2}}{4 \sqrt{3} m}\left(\mathbb{P}_{12}+\mathbb{P}_{21}\right)^{\alpha \beta} \\
& +\frac{(1-\xi)(3+\xi)}{8 \sqrt{3} m^{2}} i \not k\left(\mathbb{P}_{12}-\mathbb{P}_{21}\right)^{\alpha \beta},
\end{aligned}
$$

which reduces to Eq. (6.11) if $\xi=1$.

The invariance of matrix elements under point transformations can be discussed along the same lines. A generic interaction term for the electromagnetic coupling of the nucleon to a spin- $3 / 2$ resonance has the form

$$
\mathcal{L}_{N \Delta \gamma}=\bar{\psi}^{\alpha} \Gamma^{\alpha \mu} A^{\mu} \psi,
$$

where $\psi$ denotes the nucleon and $A^{\mu}$ the photon field. $\Gamma^{\alpha \mu}$ is the tree-level interaction vertex that satisfies $Q^{\mu} \Gamma^{\alpha \mu}=0$ and $k^{\alpha} \Gamma^{\alpha \mu}=0$ in momentum space, with $Q^{\mu}$ the photon momentum and $k^{\alpha}$ the spin-3/2 momentum. The first condition follows from electromagnetic gauge invariance and the second from spin-3/2 gauge symmetry.

Following Ref. [146], one can interpret the tree-level vertex as the special case $\Gamma^{\alpha \mu}(\xi=1)$, so that its general form becomes

$$
\Gamma^{\alpha \mu}(\xi)=\Theta^{\alpha \beta}\left(\frac{1}{\xi}\right) \Gamma^{\beta \mu}(\xi=1),
$$

and the invariance of the Lagrangian under the point transformation $(\mathrm{C} 8)$ follows from

$$
\Gamma^{\alpha \mu}(\xi)=\Theta^{\alpha \beta}\left(\frac{\xi^{\prime}}{\xi}\right) \Gamma^{\beta \mu}\left(\xi^{\prime}\right) .
$$

In combination with Eq. (C10), under a change $\xi \rightarrow \xi^{\prime}$ these transformation matrices will trivially cancel each other in any Lagrangian

$$
\mathcal{L}=\bar{\psi}^{\alpha} \Lambda^{\alpha \beta}(\xi) \psi^{\beta}+\bar{\psi}^{\alpha} \Gamma^{\alpha \mu}(\xi) A^{\mu} \psi
$$

as well as any matrix element with internal spin-3/2 legs. Since this always leads back to $\xi=1$, it is sufficient to restrict the discussion to the Rarita-Schwinger gauge.

For the correct counting of d.o.f., the invariance under point transformations is also satisfied if either the propagator or the vertex is transverse to $\gamma^{\alpha}$. Imposing this condition on the propagator by setting $\xi=0$ in Eq. (C10) has the undesired consequence that it is no longer invertible. On the other hand, it is legitimate to set $\xi \rightarrow \infty$ in Eq. (C14) because we never need to invert vertices; this is equivalent to imposing $\gamma^{\alpha} \Gamma^{\alpha \mu}=0$. In that case the interaction term is already invariant by itself and any point transformation leads to the same result:

$$
\Theta^{\alpha \beta}(\lambda) \Gamma^{\beta \mu}=\Gamma^{\alpha \mu} .
$$

Matrix elements where the internal spin-3/2 propagator is connected with two vertices are also invariant, because the relation

$$
S^{\alpha \beta}(\xi)=\Theta^{\alpha \gamma}\left(\frac{\xi}{\xi^{\prime}}\right) S^{\gamma \delta}\left(\xi^{\prime}\right) \Theta^{\delta \beta}\left(\frac{\xi}{\xi^{\prime}}\right)
$$

entails $\quad \bar{\Gamma}^{\mu \alpha} S^{\alpha \beta}(\xi) \Gamma^{\beta \nu}=\bar{\Gamma}^{\mu \alpha} S^{\alpha \beta}\left(\xi^{\prime}\right) \Gamma^{\beta \nu}$. The resulting dressed vertex admits 12 independent tensor structures, which translates to at most 12 possible independent electrocouplings in an effective Lagrangian. As discussed below Eq. (6.12), any vertex that is transverse in $k^{\alpha}$ and $\gamma^{\alpha}$ will also remove the spin- $1 / 2$ contributions from the propagators that appear in such matrix elements. In the Rarita-Schwinger gauge the condition $k^{\alpha} \Gamma^{\alpha \mu}=0$ is sufficient for this purpose because the projector $\mathbb{P}_{11}^{\alpha \beta}$ decouples from the propagator. 


\section{APPENDIX D: FORM FACTOR RELATIONS}

Here we give the relations between the constraint-free form factors $F_{i}\left(Q^{2}\right)$, which we use to parametrize the nucleon-to-resonance transition currents (with $i=1,2$ for $J^{P}=1 / 2^{ \pm}$resonances and $i=1,2,3$ for $J^{P}=3 / 2^{ \pm}$ resonances), and the experimental helicity amplitudes. We also compare with the standard form factor conventions in the literature [48,49]; see also the reviews [41,113].

We first collect the necessary definitions to arrive at compact expressions. These are the relations between the nucleon and resonance masses,

$$
\begin{aligned}
r & =\frac{m_{R}}{m}, \\
\delta & =\frac{m_{R}^{2}-m^{2}}{m^{2}}=r^{2}-1, \\
\delta_{ \pm} & =\frac{m_{R} \pm m}{2 m}=\frac{r \pm 1}{2},
\end{aligned}
$$

abbreviations for the photon momentum transfer,

$$
\tau=\frac{Q^{2}}{4 m^{2}}, \quad \tau^{\prime}=\tau-\frac{\delta}{4},
$$

and

$$
\begin{aligned}
\lambda_{ \pm} & =\frac{\left(m_{R} \pm m\right)^{2}+Q^{2}}{4 m^{2}}=\tau+\delta_{ \pm}^{2} \\
R_{ \pm} & =e \sqrt{\frac{2 \lambda_{ \pm}}{m \delta}} \\
\kappa & =\frac{\sqrt{\lambda_{+} \lambda_{-}}}{\sqrt{2} r}
\end{aligned}
$$

where $e^{2}=4 \pi \alpha_{\mathrm{em}}$ is the electric charge. Note also that $\lambda_{+} \lambda_{-}=r^{2} \tau+\tau^{\prime 2}$. In the CMS frame of the pion electroproduction process $\gamma^{*} N \rightarrow \pi N$, the Lorentz-invariant quantity

$$
2 \sqrt{2} m \kappa=\frac{2 m}{r} \sqrt{\lambda_{+} \lambda_{-}}=|\boldsymbol{k}|
$$

becomes the three-momentum of the virtual photon evaluated at the resonance position. Likewise, the threemomentum of the pion at the resonance position and for a vanishing pion mass is the so-called photon-equivalent energy: $\left|\boldsymbol{k}^{\prime}\right|_{m_{\pi}=0}=m \delta /(2 r)$.

\section{1. $J^{P}=\frac{1}{2}{ }^{ \pm}$transition form factors}

We express the on-shell $N \rightarrow 1 / 2^{ \pm}$transition matrix element in terms of Eq. (5.5),

$$
J_{\mathrm{R}}^{\mu}(k, Q)=\Lambda_{+}\left(k_{+}\right) \Gamma_{\mathrm{R}}^{\mu}(k, Q) \Lambda_{+}\left(k_{-}\right),
$$

with the onshell kinematics as in Sec. VA: $k_{-}^{\mu}$ is the nucleon momentum, $k_{+}^{\mu}$ the resonance momentum, $Q^{\mu}$ the incoming photon momentum, and $k^{\mu}=\left(k_{+}^{\mu}+k_{-}^{\mu}\right) / 2$ the average momentum of the nucleon and the resonance. On the mass shell: $k_{-}^{2}=-m^{2}, k_{+}^{2}=-m_{R}^{2}$ and therefore $k^{2}$ and $w=k \cdot Q$ are given by Eq. (5.6). The transition form factors $F_{1}\left(Q^{2}\right)$ and $F_{2}\left(Q^{2}\right)$ are defined in (5.8),

$$
\Gamma_{\mathrm{R}}^{\mu}(k, Q)=i\left[\begin{array}{c}
\mathbb{1} \\
\gamma_{5}
\end{array}\right]\left(F_{1} T_{1}^{\mu}+F_{2} \frac{T_{3}^{\mu}}{2}\right),
$$

where the $T_{i}^{\mu}$ are given in Table II and the upper (lower) entry corresponds to positive (negative) parity.

The definition in Ref. [49] is analogous but expressed in terms of two dimensionful transition form factors $G_{1}\left(Q^{2}\right)$ and $G_{2}\left(Q^{2}\right)$ :

$$
\Gamma_{\mathrm{R}}^{\mu}(k, Q)=i m^{2}\left[\begin{array}{c}
\mathbb{1} \\
\gamma_{5}
\end{array}\right]\left(G_{1} T_{1}^{\mu}+G_{2} \frac{m^{2}}{w} T_{7}^{\mu}\right) .
$$

With $T_{7}^{\mu}$ being proportional to $T_{3}^{\mu}$ on the mass shell, cf. Table V, one can read off the on-shell relations between the form factors:

$$
G_{1}=\frac{F_{1}}{m^{2}}, \quad G_{2}=\mp \frac{F_{2}}{2 m^{2} \delta_{\mp}},
$$

where upper (lower) signs correspond to resonances with positive (negative) parity.

The helicity amplitudes $A_{1 / 2}\left(Q^{2}\right)$ and $S_{1 / 2}\left(Q^{2}\right)$ are related with the form factors through [41]

$$
\begin{aligned}
& A_{1 / 2}=R_{\mp}\left(4 \tau F_{1} \pm \delta_{ \pm} F_{2}\right), \\
& S_{1 / 2}=\kappa R_{\mp}\left( \pm 4 \delta_{ \pm} F_{1}-F_{2}\right),
\end{aligned}
$$

with the inverse relations

$$
\begin{aligned}
& F_{1}=\frac{1}{4 R_{\mp} \lambda_{ \pm}}\left(A_{1 / 2} \pm \frac{\delta_{ \pm}}{\kappa} S_{1 / 2}\right), \\
& F_{2}=\frac{1}{R_{\mp} \lambda_{ \pm}}\left( \pm \delta_{ \pm} A_{1 / 2}-\frac{\tau}{\kappa} S_{1 / 2}\right) .
\end{aligned}
$$

Note that because of the factors $R_{\mp}$ and $\kappa$ the helicity amplitudes vanish either at $\lambda_{+}=0$, the pseudothreshold $\lambda_{-}=0$, or both.

\section{2. $J^{P}=\frac{3}{2}{ }^{ \pm}$transition form factors}

The onshell $N \rightarrow 3 / 2^{ \pm}$transition matrix element is given by Eq. (6.20),

$$
J_{\mathrm{R}}^{\alpha \mu}=\Lambda_{+}(k) \mathbb{P}_{3 / 2}^{\alpha \beta}(k) \Gamma_{\mathrm{R}}^{\beta \mu}(k, Q) \Lambda_{+}(k-Q),
$$


TABLE XXII. Additional tensors appearing in the $J^{P}=\frac{3}{2}^{ \pm}$ currents (D14), (D17) and (D18). For positive parity, the right column gives their onshell relations which relate them to $T_{1}, T_{2}$ and $T_{3}$ defined in Table VII. For negative parity the same relations hold if one exchanges $r \rightarrow-r$ and $\lambda_{-} \rightarrow \lambda_{+}$.

\begin{tabular}{ll}
\hline \hline$m^{4} T_{21}^{\alpha \mu}=\varepsilon_{k Q}^{\alpha \beta} \varepsilon_{k Q}^{\beta \mu}$ & $T_{21}-2 \tau^{\prime} T_{2}-r T_{3}$ \\
$m^{4} T_{22}^{\alpha \mu}=Q^{\alpha} k^{\beta} t_{Q Q}^{\beta \mu}$ & $r T_{22}+4 r \tau T_{2}-2 \tau^{\prime} T_{3}$ \\
$m T_{23}^{\alpha \mu}=-i t_{\gamma Q}^{\alpha \mu}$ & $r T_{23}-T_{1}-T_{2}$ \\
$m^{2} T_{24}^{\alpha \mu}=t_{Q Q}^{\alpha \mu}$ & $r T_{24}-T_{3}$ \\
$m^{4} T_{25}^{\alpha \mu}=\not k Q^{\alpha} \gamma^{\beta} \varepsilon_{k Q}^{\beta \mu}$ & $T_{25}+2 \lambda_{-} T_{1}+2 \tau^{\prime} T_{2}+r T_{3}$ \\
\hline \hline
\end{tabular}

where $k$ is the outgoing momentum of the resonance and $Q$ is the incoming photon momentum. On the mass shell: $(k-Q)^{2}=-m^{2}$ and $k^{2}=-m_{R}^{2}$, which entails $k \cdot Q=2 m^{2} \tau^{\prime}$. The constraint-free transition form factors $F_{i}\left(Q^{2}\right)$ are defined via (6.24),

$$
\Gamma_{\mathrm{R}}^{\alpha \mu}(k, Q)=\left[\begin{array}{c}
\gamma_{5} \\
\mathbb{1}
\end{array}\right]\left(F_{1} T_{1}^{\alpha \mu}-F_{2} T_{2}^{\alpha \mu}-F_{3} T_{3}^{\alpha \mu}\right),
$$

where the upper (lower) entry corresponds to positive (negative) parity. The $T_{i}^{\alpha \mu}$ are defined in Table VII.

To write down the various different versions of the onshell currents used in the literature, we define the tensors $T_{21} \ldots T_{25}$ in Table XXII in addition to those in Table VII. On the mass shell and inside the positive-energy and RaritaSchwinger projectors (D11) they are linearly related with $T_{1}, T_{2}$ and $T_{3}$, but with the exception of $T_{21}$ they do not satisfy the off-shell constraint $k^{\alpha} T_{i}^{\alpha \mu}=0$.

Following [41], the experimentally extracted helicity amplitudes $A_{3 / 2}\left(Q^{2}\right), A_{1 / 2}\left(Q^{2}\right)$ and $S_{1 / 2}\left(Q^{2}\right)$ are related to the helicity form factors $h_{i}\left(Q^{2}\right)$ via

$$
\left\{h_{1}, h_{2}, h_{3}\right\}=\sqrt{\frac{3}{2}} \frac{4}{R_{\mp}}\left\{\frac{r S_{1 / 2}}{2 \kappa}, \pm \frac{A_{3 / 2}}{\sqrt{3}}, A_{1 / 2}\right\}
$$

where upper (lower) signs denote positive (negative) parity. The corresponding form of the current is [49]

$$
\begin{aligned}
\Gamma_{\mathrm{R}}^{\alpha \mu}= & \frac{1}{16 \lambda_{+} \lambda_{-}}\left[\begin{array}{c}
\gamma_{5} \\
\mathbb{1}
\end{array}\right]\left(-h_{1} T_{22}^{\alpha \mu}+2 h_{2} T_{21}^{\alpha \mu}\right. \\
& \left.+\left(h_{2}+h_{3}\right) T_{25}^{\alpha \mu}\right)
\end{aligned}
$$

which is neither free of kinematics nor satisfies the off-shell constraints. Using the on-shell relations in Table XXII, the helicity form factors (and thus helicity amplitudes) are related to the $F_{i}$ via

$$
\begin{aligned}
& F_{1}=-\frac{h_{2}+h_{3}}{8 \lambda_{ \pm}} \\
& F_{2}=-\frac{1}{8 \lambda_{+} \lambda_{-}}\left[2 \tau h_{1}+\tau^{\prime}\left(h_{2}-h_{3}\right)\right] \\
& F_{3}= \pm \frac{1}{8 r \lambda_{+} \lambda_{-}}\left[\tau^{\prime} h_{1}-\frac{r^{2}}{2}\left(h_{2}-h_{3}\right)\right]
\end{aligned}
$$

and vice versa

$$
\begin{aligned}
& h_{1}=-4\left(r^{2} F_{2} \mp 2 r \tau^{\prime} F_{3}\right), \\
& h_{2}=-4\left(\lambda_{ \pm} F_{1}+\tau^{\prime} F_{2} \pm 2 r \tau F_{3}\right), \\
& h_{3}=-4\left(\lambda_{ \pm} F_{1}-\tau^{\prime} F_{2} \mp 2 r \tau F_{3}\right) .
\end{aligned}
$$

Also here the helicity amplitudes in Eq. (D13) vanish either at $\lambda_{+}=0$ or $\lambda_{-}=0$ due to the factors $R_{\mp}$.

Another form of the current expressed in terms of three form factors $G_{i}\left(Q^{2}\right)$ is $[41,49]$ :

$$
\Gamma_{\mathrm{R}}^{\alpha \mu}=\left[\begin{array}{c}
\gamma_{5} \\
\mathbb{1}
\end{array}\right]\left(G_{1} T_{23}^{\alpha \mu}+G_{2} T_{2}^{\alpha \mu}+G_{3} T_{24}^{\alpha \mu}\right) .
$$

Here we defined the $G_{i}$ to be dimensionless (in the standard definition they carry dimensions: replace $G_{1} \rightarrow m G_{1}$ and $G_{2,3} \rightarrow m^{2} G_{2,3}$.) They are free of kinematics but again the current does not satisfy the off-shell constraints. Their relation with the $F_{i}$ is

$$
G_{1}= \pm r F_{1}, \quad G_{2}=-\left(F_{1}+F_{2}\right), \quad G_{3}=\mp r F_{3} .
$$

Finally, the Jones-Scadron form of the current in terms of $G_{E}^{*}, G_{M}^{*}$ and $G_{C}^{*}$ is given by $[48,49]$

$$
\begin{aligned}
\Gamma_{\mathrm{R}}^{\alpha \mu}= & \pm \sqrt{\frac{3}{2} \frac{\delta_{ \pm}}{2 \lambda_{+} \lambda_{-}}}\left[\begin{array}{c}
\gamma_{5} \\
\mathbb{1}
\end{array}\right]\left(\left[\begin{array}{c}
G_{M}^{*}-G_{E}^{*} \\
2 G_{M}^{*}
\end{array}\right] \lambda_{\mp} T_{1}^{\alpha \mu}\right. \\
& \left.+\left[\begin{array}{c}
-2 G_{E}^{*} \\
G_{M}^{*}-G_{E}^{*}
\end{array}\right] \frac{T_{21}^{\alpha \mu}}{2} \mp G_{C}^{*} \frac{T_{22}^{\alpha \mu}}{2}\right) .
\end{aligned}
$$

As before, upper (lower) components and signs denote positive (negative) parity. The Jones-Scadron form factors are related to the helicity form factors via

$$
\begin{aligned}
{\left[\begin{array}{c}
G_{M}^{*} \\
G_{E}^{*}
\end{array}\right] } & =-\sqrt{\frac{3}{2}} \frac{h_{3} \pm 3 h_{2}}{12 \delta_{ \pm}}, \\
{\left[\begin{array}{c}
G_{E}^{*} \\
G_{M}^{*}
\end{array}\right] } & =\sqrt{\frac{3}{2}} \frac{h_{3} \mp h_{2}}{12 \delta_{ \pm}}, \\
G_{C}^{*} & =\sqrt{\frac{3}{2}} \frac{h_{1}}{6 \delta_{ \pm}} .
\end{aligned}
$$

This coincides with the conventions in $[41,48]$ whereas in Ref. [49] $G_{M}^{*}, G_{E}^{*}$ and $G_{C}^{*}$ are defined without the factor $\sqrt{3 / 2}$ on the r.h.s. The relations between the JonesScadron form factors and the $F_{i}$ are given by 


$$
\begin{aligned}
G_{M}^{*} & =\sqrt{\frac{2}{3}} \frac{1}{\delta_{ \pm}}\left[\begin{array}{c}
2 \lambda_{+} F_{1}+\tau^{\prime} F_{2}+2 r \tau F_{3} \\
-\lambda_{-} F_{1}
\end{array}\right], \\
G_{E}^{*} & =\sqrt{\frac{2}{3}} \frac{1}{\delta_{ \pm}}\left[\begin{array}{c}
\tau^{\prime} F_{2}+2 r \tau F_{3} \\
-\lambda_{-} F_{1}-2 \tau^{\prime} F_{2}+4 r \tau F_{3}
\end{array}\right], \\
G_{C}^{*} & =\sqrt{\frac{2}{3}} \frac{1}{\delta_{ \pm}}\left(-r^{2} F_{2} \pm 2 r \tau^{\prime} F_{3}\right)
\end{aligned}
$$

$$
\begin{aligned}
& F_{1}=\sqrt{\frac{3}{2}} \frac{\delta_{ \pm}}{2 \lambda_{+} \lambda_{-}}\left[\begin{array}{c}
\lambda_{-}\left(G_{M}^{*}-G_{E}^{*}\right) \\
-2 \lambda_{+} G_{M}^{*}
\end{array}\right], \\
& F_{2}=\sqrt{\frac{3}{2} \frac{\delta_{ \pm}}{\lambda_{+} \lambda_{-}}}\left[\begin{array}{c}
\tau^{\prime} G_{E}^{*}-\tau G_{C}^{*} \\
\frac{1}{2} \tau^{\prime}\left(G_{M}^{*}-G_{E}^{*}\right)-\tau G_{C}^{*}
\end{array}\right], \\
& F_{3}=\sqrt{\frac{3}{2}} \frac{\delta_{ \pm}}{2 r \lambda_{+} \lambda_{-}}\left[\begin{array}{c}
r^{2} G_{E}^{*}+\tau^{\prime} G_{C}^{*} \\
-\frac{1}{2} r^{2}\left(G_{M}^{*}-G_{E}^{*}\right)-\tau^{\prime} G_{C}^{*}
\end{array}\right] .
\end{aligned}
$$

and vice versa

[1] D. Drechsel, B. Pasquini, and M. Vanderhaeghen, Phys. Rep. 378, 99 (2003).

[2] M. Schumacher, Prog. Part. Nucl. Phys. 55, 567 (2005).

[3] H. Griesshammer, J. McGovern, D. Phillips, and G. Feldman, Prog. Part. Nucl. Phys. 67, 841 (2012).

[4] B. R. Holstein and S. Scherer, Annu. Rev. Nucl. Part. Sci. 64, 51 (2014).

[5] F. Hagelstein, R. Miskimen, and V. Pascalutsa, Prog. Part. Nucl. Phys. 88, 29 (2016).

[6] P. A. Guichon, G. Liu, and A. W. Thomas, Nucl. Phys. A591, 606 (1995).

[7] D. Drechsel, G. Knochlein, A. Y. Korchin, A. Metz, and S. Scherer, Phys. Rev. C 58, 1751 (1998).

[8] E. Downie and H. Fonvieille, Eur. Phys. J. Spec. Top. 198, 287 (2011).

[9] V. Lensky, V. Pascalutsa, and M. Vanderhaeghen, Eur. Phys. J. C 77, 119 (2017).

[10] A. V. Belitsky, D. Mueller, and A. Kirchner, Nucl. Phys. B629, 323 (2002).

[11] A. V. Belitsky and A. V. Radyushkin, Phys. Rep. 418, 1 (2005).

[12] M. Guidal, H. Moutarde, and M. Vanderhaeghen, Rep. Prog. Phys. 76, 066202 (2013).

[13] K. Kumericki, S. Liuti, and H. Moutarde, Eur. Phys. J. A 52, 157 (2016).

[14] P. A. M. Guichon and M. Vanderhaeghen, Phys. Rev. Lett. 91, 142303 (2003).

[15] C. E. Carlson and M. Vanderhaeghen, Annu. Rev. Nucl. Part. Sci. 57, 171 (2007).

[16] J. Arrington, P. Blunden, and W. Melnitchouk, Prog. Part. Nucl. Phys. 66, 782 (2011).

[17] R. Pohl et al., Nature (London) 466, 213 (2010).

[18] M. C. Birse and J. A. McGovern, Eur. Phys. J. A 48, 120 (2012).

[19] A. Antognini et al., Science 339, 417 (2013).

[20] A. Antognini, F. Kottmann, F. Biraben, P. Indelicato, F. Nez, and R. Pohl, Ann. Phys. (Amsterdam) 331, 127 (2013).

[21] R. Pohl, R. Gilman, G. A. Miller, and K. Pachucki, Annu. Rev. Nucl. Part. Sci. 63, 175 (2013).

[22] C. E. Carlson, Prog. Part. Nucl. Phys. 82, 59 (2015).

[23] V. Bernard, N. Kaiser, A. Schmidt, and U. G. Meissner, Phys. Lett. B 319, 269 (1993).
[24] T. R. Hemmert, B. R. Holstein, and J. Kambor, Phys. Rev. D 55, 5598 (1997).

[25] V. Lensky and V. Pascalutsa, Eur. Phys. J. C 65, 195 (2010).

[26] G. Eichmann and C. S. Fischer, Phys. Rev. D 85, 034015 (2012).

[27] G. Eichmann and C. S. Fischer, Phys. Rev. D 87, 036006 (2013).

[28] G. Eichmann, H. Sanchis-Alepuz, R. Williams, R. Alkofer, and C. S. Fischer, Prog. Part. Nucl. Phys. 91, 1 (2016).

[29] R. Tarrach, Nuovo Cimento A 28, 409 (1975).

[30] W. A. Bardeen and W. Tung, Phys. Rev. 173, 1423 (1968).

[31] D. Drechsel, G. Knochlein, A. Y. Korchin, A. Metz, and S. Scherer, Phys. Rev. C 57, 941 (1998).

[32] M. Gorchtein, Phys. Rev. C 81, 015206 (2010).

[33] D. Drechsel, G. Knochlein, A. Metz, and S. Scherer, Phys. Rev. C 55, 424 (1997).

[34] B. L. G. Bakker and C.-R. Ji, Few-Body Syst. 58, 8 (2017).

[35] C. de Calan and R. Stora, CERN Report No. TH-1004, 1969.

[36] V. Pascalutsa and D. R. Phillips, Phys. Rev. C 67, 055202 (2003).

[37] V. Pascalutsa and D. R. Phillips, Phys. Rev. C 68, 055205 (2003).

[38] S. Kondratyuk, P. G. Blunden, W. Melnitchouk, and J. A. Tjon, Phys. Rev. Lett. 95, 172503 (2005).

[39] S. Kondratyuk and P. G. Blunden, Phys. Rev. C 75, 038201 (2007).

[40] X. Cao and H. Lenske, Phys. Lett. B 772, 274 (2017).

[41] I. G. Aznauryan and V. D. Burkert, Prog. Part. Nucl. Phys. 67, 1 (2012).

[42] L. Tiator, D. Drechsel, S. S. Kamalov, and M. Vanderhaeghen, Eur. Phys. J. Spec. Top. 198, 141 (2011).

[43] V. I. Mokeev, I. Aznauryan, V. Burkert, and R. Gothe, EPJ Web Conf. 113, 01013 (2016).

[44] V. I. Mokeev et al., Phys. Rev. C 93, 025206 (2016).

[45] V. I. Mokeev, Few-Body Syst. 59, 46 (2018).

[46] S. Weinberg and E. Witten, Phys. Lett. 96B, 59 (1980).

[47] V. Pascalutsa and R. Timmermans, Phys. Rev. C 60, 042201 (1999).

[48] H. F. Jones and M. D. Scadron, Ann. Phys. (N.Y.) 81, 1 (1973). 
[49] R. C. E. Devenish, T. S. Eisenschitz, and J. G. Korner, Phys. Rev. D 14, 3063 (1976).

[50] G. Eichmann, C. S. Fischer, and W. Heupel, Phys. Rev. D 92, 056006 (2015).

[51] A. I. L’vov, Sov. J. Nucl. Phys. 34, 597 (1981).

[52] A. L'vov, V. Petrun'kin, and M. Schumacher, Phys. Rev. C 55, 359 (1997).

[53] V. Bernard, N. Kaiser, and U. G. Meissner, Phys. Rev. Lett. 67, 1515 (1991).

[54] V. Bernard, N. Kaiser, and U.-G. Meissner, Int. J. Mod. Phys. E 04, 193 (1995).

[55] H. W. Fearing and S. Scherer, Few-Body Syst. 23, 111 (1998).

[56] A. Kizilersu, M. Reenders, and M. Pennington, Phys. Rev. D 52, 1242 (1995).

[57] J. Skullerud and A. Kizilersu, J. High Energy Phys. 09 (2002) 013.

[58] J. S. Ball and T.-W. Chiu, Phys. Rev. D 22, 2542 (1980).

[59] S. Scherer, A. Y. Korchin, and J. Koch, Phys. Rev. C 54, 904 (1996).

[60] J. Friedrich and T. Walcher, Eur. Phys. J. A 17, 607 (2003).

[61] G. Eichmann, R. Williams, R. Alkofer, and M. Vujinovic, Phys. Rev. D 89, 105014 (2014).

[62] G. Eichmann, Acta Phys. Pol. B Proc. Suppl. 7, 597 (2014).

[63] F. E. Low, Phys. Rev. 96, 1428 (1954).

[64] M. Gell-Mann and M. Goldberger, Phys. Rev. 96, 1433 (1954).

[65] L. M. Nath, B. Etemadi, and J. D. Kimel, Phys. Rev. D 3, 2153 (1971).

[66] C. R. Hagen, Phys. Rev. D 4, 2204 (1971).

[67] L. P. S. Singh, Phys. Rev. D 7, 1256 (1973).

[68] M. Benmerrouche, R. M. Davidson, and N.C. Mukhopadhyay, Phys. Rev. C 39, 2339 (1989).

[69] V. Pascalutsa, Phys. Rev. D 58, 096002 (1998).

[70] P. Van Nieuwenhuizen, Phys. Rep. 68, 189 (1981).

[71] S. Kondratyuk and O. Scholten, Phys. Rev. C 64, 024005 (2001).

[72] F. Hagelstein, Few-Body Syst. 59, 93 (2018).

[73] I. G. Aznauryan et al., Phys. Rev. C 80, 055203 (2009).

[74] V. I. Mokeev et al., Phys. Rev. C 86, 035203 (2012).

[75] V. Mokeev, https://userweb.jlab.org/ mokeev/resonance_ electrocouplings/.

[76] D. Drechsel, S. S. Kamalov, and L. Tiator, Eur. Phys. J. A 34, 69 (2007).

[77] L. Tiator, D. Drechsel, S. S. Kamalov, and M. Vanderhaeghen, Chin. Phys. C 33, 1069 (2009).

[78] C. Patrignani and Olive K. A. (Particle Data Group), Chin. Phys. C 40, 100001 (2016).

[79] G. P. Lepage and S. J. Brodsky, Phys. Rev. Lett. 43, 545 (1979); 43, 1625(E) (1979).

[80] S. J. Brodsky and G. P. Lepage, Phys. Rev. D 24, 2848 (1981).

[81] C. E. Carlson, Phys. Rev. D 34, 2704 (1986).

[82] C. E. Carlson and N. C. Mukhopadhyay, Phys. Rev. Lett. 81, 2646 (1998).

[83] S. Stajner et al., Phys. Rev. Lett. 119, 022001 (2017).
[84] K. Park et al. (CLAS Collaboration), Phys. Rev. C 91, 045203 (2015).

[85] V. I. Mokeev and I. G. Aznauryan, Int. J. Mod. Phys. Conf. Ser. 26, 1460080 (2014).

[86] V. V. Frolov et al., Phys. Rev. Lett. 82, 45 (1999).

[87] A. N. Villano et al., Phys. Rev. C 80, 035203 (2009).

[88] N. Sparveris et al., Eur. Phys. J. A 49, 136 (2013).

[89] A. Blomberg et al., Phys. Lett. B 760, 267 (2016).

[90] A. J. F. Siegert, Phys. Rev. 52, 787 (1937).

[91] D. Drechsel and L. Tiator, J. Phys. G 18, 449 (1992).

[92] L. Tiator, Few-Body Syst. 57, 1087 (2016).

[93] G. Ramalho, Phys. Lett. B 759, 126 (2016).

[94] I. G. Aznauryan, Phys. Rev. C 76, 025212 (2007).

[95] H.-W. Lin, S. D. Cohen, R. G. Edwards, and D. G. Richards, Phys. Rev. D 78, 114508 (2008).

[96] I. T. Obukhovsky, A. Faessler, D. K. Fedorov, T. Gutsche, and V. E. Lyubovitskij, Phys. Rev. D 84, 014004 (2011).

[97] T. Gutsche, V. E. Lyubovitskij, I. Schmidt, and A. Vega, Phys. Rev. D 87, 016017 (2013).

[98] T. Bauer, S. Scherer, and L. Tiator, Phys. Rev. C 90, 015201 (2014).

[99] J. Segovia, B. El-Bennich, E. Rojas, I. C. Cloet, C. D. Roberts, S.-S. Xu, and H.-S. Zong, Phys. Rev. Lett. 115, 171801 (2015).

[100] G. Ramalho and D. Melnikov, Phys. Rev. D 97, 034037 (2018).

[101] G. Ramalho and M. T. Peña, Phys. Rev. D 84, 033007 (2011).

[102] G. Ramalho and K. Tsushima, Phys. Rev. D 84, 051301 (2011).

[103] G. Ramalho, D. Jido, and K. Tsushima, Phys. Rev. D 85, 093014 (2012).

[104] G. Ramalho, Phys. Rev. D 95, 054008 (2017).

[105] G. Ramalho, Phys. Rev. D 90, 033010 (2014).

[106] G. Ramalho, Phys. Rev. D 93, 113012 (2016).

[107] N. F. Sparveris et al. (OOPS Collaboration), Phys. Rev. Lett. 94, 022003 (2005).

[108] G. Ramalho, M. T. Pena, and F. Gross, Eur. Phys. J. A 36, 329 (2008).

[109] G. Ramalho, M. T. Pena, and F. Gross, Phys. Rev. D 78, 114017 (2008).

[110] B. Julia-Diaz, T. S. H. Lee, A. Matsuyama, T. Sato, and L. C. Smith, Phys. Rev. C 77, 045205 (2008).

[111] B. Julia-Diaz, H. Kamano, T. S. H. Lee, A. Matsuyama, T. Sato, and N. Suzuki, Phys. Rev. C 80, 025207 (2009).

[112] V. D. Burkert and T. S. H. Lee, Int. J. Mod. Phys. E 13, 1035 (2004).

[113] V. Pascalutsa, M. Vanderhaeghen, and S. N. Yang, Phys. Rep. 437, 125 (2007).

[114] G. Ramalho and M. T. Pena, Phys. Rev. D 80, 013008 (2009).

[115] V. Pascalutsa and M. Vanderhaeghen, Phys. Rev. D 76, 111501 (2007).

[116] A. J. Buchmann, Phys. Rev. Lett. 93, 212301 (2004).

[117] G. Ramalho, Eur. Phys. J. A 54, 75 (2018).

[118] G. Eichmann and D. Nicmorus, Phys. Rev. D 85, 093004 (2012). 
[119] J. Segovia, I. C. Cloet, C. D. Roberts, and S. M. Schmidt, Few-Body Syst. 55, 1185 (2014).

[120] H. Sanchis-Alepuz, R. Alkofer, and C. S. Fischer, Eur. Phys. J. A 54, 41 (2018).

[121] G. Ramalho, Phys. Rev. D 94, 114001 (2016).

[122] G. Ramalho and M. T. Peña, Phys. Rev. D 89, 094016 (2014).

[123] G. Ramalho and M. T. Peña, Phys. Rev. D 95, 014003 (2017).

[124] R. Koniuk and N. Isgur, Phys. Rev. D 21, 1868 (1980); 23, 818(E) (1981).

[125] E. Santopinto and M. M. Giannini, Phys. Rev. C 86, 065202 (2012).

[126] P. P. Martel et al. (A2 Collaboration), Phys. Rev. Lett. 114, 112501 (2015).

[127] B. Pasquini, P. Pedroni, and D. Drechsel, Phys. Lett. B 687, 160 (2010).

[128] M. Camen et al., Phys. Rev. C 65, 032202 (2002).

[129] A. Baldin, Nucl. Phys. 18, 310 (1960).

[130] B. R. Holstein, D. Drechsel, B. Pasquini, and M. Vanderhaeghen, Phys. Rev. C 61, 034316 (2000).

[131] D. Babusci, G. Giordano, A. I. L'vov, G. Matone, and A. M. Nathan, Phys. Rev. C 58, 1013 (1998).

[132] G. C. Gellas, T. R. Hemmert, and U.-G. Meissner, Phys. Rev. Lett. 85, 14 (2000).

[133] K. B. Vijaya Kumar, J. A. McGovern, and M. C. Birse, Phys. Lett. B 479, 167 (2000).
[134] T. R. Hemmert, B. R. Holstein, J. Kambor, and G. Knochlein, Phys. Rev. D 57, 5746 (1998).

[135] J. A. McGovern, D. R. Phillips, and H. W. Griesshammer, Eur. Phys. J. A 49, 12 (2013).

[136] V. Lensky, J. McGovern, and V. Pascalutsa, Eur. Phys. J. C 75, 604 (2015).

[137] A. Gasparyan, M. Lutz, and B. Pasquini, Nucl. Phys. A866, 79 (2011).

[138] E. D. Bloom and F. J. Gilman, Phys. Rev. Lett. 25, 1140 (1970).

[139] W. Melnitchouk, R. Ent, and C. Keppel, Phys. Rep. 406, 127 (2005).

[140] R. Ent, in Electromagnetic Interactions and Hadronic Structure, edited by F. Close et al. (Cambridge University Press, Cambridge, UK, 2007), pp. 424-456, http:// inspirehep.net/record/761211? ln=en.

[141] G. Eichmann, C. Fischer, E. Weil, and R. Williams, Phys. Lett. B 774, 425 (2017).

[142] E. Weil, G. Eichmann, C. S. Fischer, and R. Williams, Phys. Rev. D 96, 014021 (2017).

[143] N. Krupina, V. Lensky, and V. Pascalutsa, Phys. Lett. B 782, 34 (2018).

[144] G. Miller, A. Thomas, J. Carroll, and J. Rafelski, Phys. Rev. A 84, 020101 (2011).

[145] H. Haberzettl, arXiv:nucl-th/9812043.

[146] V. Shklyar and H. Lenske, Phys. Rev. C 80, 058201 (2009). 